\title{
A Hybrid Systems Model for Simple Manipulation and Self-Manipulation Systems
}

\author{
Aaron M. Johnson* $\quad$ Samuel A. Burden $\quad$ D. E. Koditschek
}

\begin{abstract}
Rigid bodies, plastic impact, persistent contact, Coulomb friction, and massless limbs are ubiquitous simplifications introduced to reduce the complexity of mechanics models despite the obvious physical inaccuracies that each incurs individually. In concert, it is well known that the interaction of such idealized approximations can lead to conflicting and even paradoxical results. As robotics modeling moves from the consideration of isolated behaviors to the analysis of tasks requiring their composition, a mathematically tractable framework for building models that combine these simple approximations yet achieve reliable results is overdue. In this paper we present a formal hybrid dynamical system model that introduces suitably restricted compositions of these familiar abstractions with the guarantee of consistency analogous to global existence and uniqueness in classical dynamical systems. The hybrid system developed here provides a discontinuous but self-consistent approximation to the continuous (though possibly very stiff and fast) dynamics of a physical robot undergoing intermittent impacts. The modeling choices sacrifice some quantitative numerical efficiencies while maintaining qualitatively correct and analytically tractable results with consistency guarantees promoting their use in formal reasoning about mechanism, feedback control, and behavior design in robots that make and break contact with their environment.
\end{abstract}

\section{Introduction}

Simple models of complex robot-world interactions are key to understanding, implementing and generalizing behaviors as well as identifying and composing their reusable constituents to generate new behaviors (Full and Koditschek 1999). There is strong appeal to using familiar physical simplifications such as rigid bodies, plastic impacts, persistent contact, Coulomb friction, and massless limbs in building up simple robotics models. Their coarse approximation to the underlying physical processes of interest are widely understood to offer the right combination of analytical tractability and physical realism in isolation. However, it is also widely understood that such individually useful simplifications can introduce catastrophic side-effects when combined (e.g. in Chatterjee (1999), Dupont and Yamajako (1994), Keller (1986), Mason and Wang (1988), Painlevé (1895), Trinkle et al. (1997) and others, as discussed in Section 1.3).

In this paper we assemble a framework of reasonable physical assumptions and accompanying mechanics to develop a formalism for combining them at will in the construction of a simple hybrid system model for contact robotics that yields a provably consistent ${ }^{1}$ and empirically useful approximation to many behavioral settings of interest. As an example of the value of such mathematical models, new work (Brill et al. 2015) uses the formal properties of our self-manipulation model to develop rigorous correctness (or, non-existence) proofs for desirable robot behaviors - in that case, gap crossing and ledge mounting. However, while the primary goal of this paper is not numerical analysis, simulation does provide a useful way to visualize key features of the model and the utility of some of the simplifying assumptions. Numerical results obtained through a custom Mathematica simulation (described in Section 4.4) are used throughout the paper to illustrate key concepts, and to suggest the fidelity to physical settings of interest.

For example, our model generates simulations ${ }^{2}$ of the leaping behavior depicted in Figure 1 that recreate the empirical results of Johnson and Koditschek (2013b) qualitatively (i.e., predicts the same salient features though not

\footnotetext{
${ }^{*}$ Corresponding author; Robotics Institute, Carnegie Mellon, Pittsburgh, PA, USA, e-mail: amj1 @ andrew.cmu.edu

${ }^{1}$ Here, consistent refers to a combination of properties detailed in Section 3.4 analogous to the guarantee of global existence and uniqueness of solutions for a classical dynamical system.

${ }^{2}$ For this simulation the middle and rear legs are used with a maximum current limit of 20A, a pseudo-impulse (defined in Section 2.7) magnitude of $\delta_{t}=0.03$ (hand selected to give the qualitatively best overall results), relative leg timing of $t_{2}=0.01$ (i.e., the middle legs are started $0.01 \mathrm{~s}$ before the rear legs), and once a leg has lifted off the ground it is slowly rotated upwards out of the way. Remaining model parameters are as listed in (Johnson and Koditschek 2013a, Sec. III, Appendix G).
} 


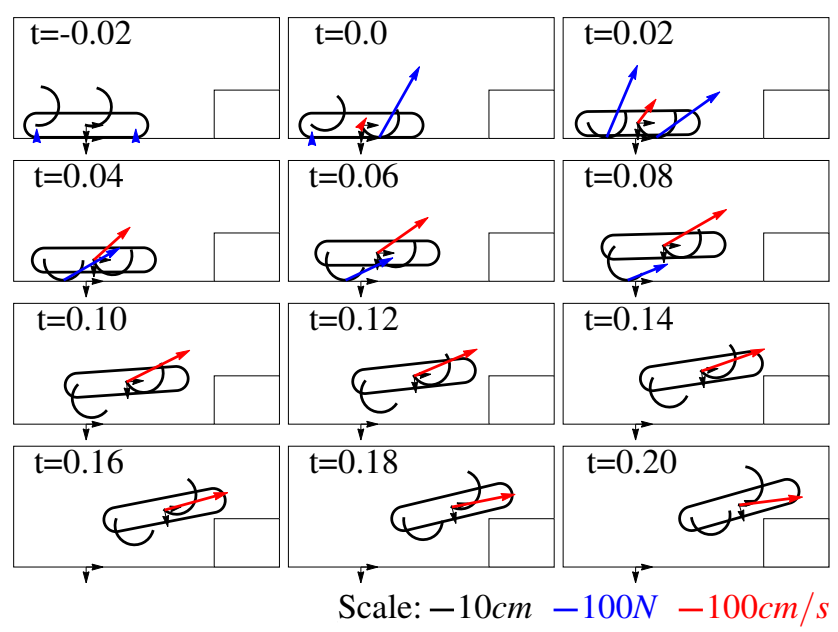

Figure 1: Keyframes from RHex simulation leaping onto a $20 \mathrm{~cm}$ ledge. Blue arrows show contact forces while the red arrow shows body velocity.

necessarily the same metric results), yet enjoys a combination of mathematical properties that we believe will provide a foundation for reasoning about and thereby generalizing the platform design and control strategies that gave rise to such behaviors. Of course, physical fidelity is not mathematically demonstrable and the relevance of the modeling choices we propose (i.e., the empirical sway of this formally self-consistent model) can only be established over the long run in practice by the breadth of physical phenomena they usefully approximate, regardless of the simplification and ease of analysis they afford.

The paper is structured as follows. This section finishes with a summary of contributions, followed by a discussion of their relation to prior work. Section 2 introduces the various simplifying physical modeling assumptions and draws out some of the mathematical consequences bearing on their relationships to alternative formulations and to each other. Section 3 assembles from these pieces a formal hybrid dynamical system model and proves its consistency. Section 4 reviews the scope of physical settings admitted by our assumptions and discusses the most delicate aspects of their interplay with our formal results, providing additional examples that help give a broader context for the applicability of the theory. Section 5 concludes with some final thoughts on the implications of this work and future directions. An extensive Appendix works through the details of selected proofs and provides additional background material.

\subsection{Contributions of the Paper}

This paper extends a framework for manipulation (Murray et al. 1994) and self-manipulation (Johnson and Koditschek 2013a) modeling into a formal hybrid dynamical systems specification whose discrete modes are indexed by the active contact constraint set in a manner guaranteed to produce a unique execution from every initial condition under mild conditions on the motor feedback control laws. The foundation on which we rest this physically simple and mathematically tractable modeling framework arises from Assumptions A1-A12, introduced in Section 2 and discussed further in Section 4.1, comprising various familiar phenomenological representations and physically natural hypotheses, including: rigid bodies (A1), massless limbs (A4), plastic impact (A8), and static friction (A12). It is known that in general these properties are not mutually consistent, however we formally demonstrate that the particular set of assumptions included here provides a well defined, deterministic, and computationally well-behaved model. The physical fidelity may, in some important applications contexts that we point out, necessarily remain something of a leap of faith relative to the still incomplete state of the theory of rigid body mechanics. To the best of our knowledge this is the first time any succinctly stated list of physical assumptions about rigid body mechanics has been shown to yield a consistent hybrid dynamical system with unique and globally defined executions.

Our central technical contribution is the derivation of a consistent extension of Lagrangian dynamics, Newtonian impact laws, and complementarity contact conditions to systems that have certain rank deficiencies in their inertia tensor that agrees with (i.e., when rank is restored, maintains equivalence to) the nonsingular case (Lemmas 4, 5, \& 8 and Theorems $1 \& 2$ ). The possibly massless dynamics motivate a reformulation of complementarity as a logical equiv- 
alence (Lemma 9) so that its unique solvability (for both force-acceleration and impulse-velocity complementarity problems, Assumptions A9 \& A10, respectively) is shown to imply a unique partition of the guard set (i.e., those states which are to undergo a mode transition) into disjoint components labeled deterministically by the destination mode of the transition (Theorem 5). These conditions are expressed in terms of a higher order scalar relation $(\prec$, Definition 1$)$, and we exhibit certain properties of this relation that clarify its role in determining the guard set (Lemmas 1-3).

Even without the introduction of massless limbs there exist many opportunities for repeated (and even Zeno ${ }^{3}$ ) discrete transitions that seem unlikely to add much physical insight (and, speaking practically, generally degrade the numerical performance of simulations based upon this model). Hence, to resolve the qualitative problem of spurious transitions at arbitrarily low velocities (Lemma 10), we introduce a new pseudo-impulse, which acts on the discrete transitional logic (rather than the continuous dynamics), imposing an implicit bound on contact velocity below which such contacts persist (Theorem 3), precluding certain Zeno phenomena (Theorem 11).

As a structure to combine these physical models and assumptions, this work presents the formal definition of the self-manipulation hybrid system in Definition 5 (along with Definitions 2-4), and the formal demonstration of its consistency (including that it is deterministic and non-blocking, Theorems 4-9 and Lemmas 6 \& 7), incorporating a well-behaved notion of completion in case of a Zeno execution (Definition 6, Theorem 10, Corollary 1) by adapting to this more elementary setting the measure theoretic arguments of Ballard (2000).

\subsection{Reader's Guide}

We anticipate that different readers will approach this paper with diverse goals and backgrounds. In this section we suggest sections that may have the greatest relevance with respect to specific interests. All readers are encouraged to start with the setup and notation explained in Section 2.1 and summarized in Tables 1 and 2.

- For readers interested in instantiating a hybrid dynamical system model of a specific robot, the most relevant section is Section 3.2, which is based on the assumptions and derivations in Section 2 and definitions in Section 3.1. The continuous dynamics in any particular contact mode is based on Murray et al. (1994) for manipulation and Johnson and Koditschek (2013a) for self-manipulation systems (whose differences are summarized in Section 2.2). See also the discussion on numerical simulation of this system in Section 4.4.

- Some readers may be interested in the relationship of this model to the still evolving theory of rigid body mechanics. They will likely wish to focus on our treatment of: massless bodies and singular inertia tensors (Section 2.3, with implications throughout Section 2 and in Section 4.2), Lagrangian dynamics (Section 2.4), impulsive dynamics (Sections 2.5, 2.7, and 4.3), complementarity systems (Section 2.6), friction (Section 2.8), and accumulation points (Section 3.5). See also the discussion in Section 4.1 on these mechanics assumptions.

- Those most interested in the properties of this model considered as a mathematical object will likely wish to focus on the hybrid dynamical system itself which is presented in Sections 3.1 and 3.2, with various properties related to existence and uniqueness of executions proven in Sections 3.3 and 3.4. Zeno considerations are presented in Section 3.5, based in part on the pseudo-impulse presented in Section 2.7, and discussed further in Section 4.5.

- Readers interested in the particular example of the RHex robot used in, e.g., Figure 1 can find details on the model (lengths, masses, etc) in Johnson and Koditschek (2013a) and details on the behaviors this paper aims to model in Johnson and Koditschek (2013b). Of particular interest for modeling similar robots may be the treatment of massless legs (Sections 2.3 and 4.2), the pseudo-impulse (Section 2.7, in particular the example in Section 2.7.2), and the full self-manipulation hybrid system model (Section 3.2).

\subsection{Relation to Prior Literature}

This paper aims to promote simplified physics based models of robotic systems for purposes of analysis. Doing so entails integrating results and ideas that have developed somewhat independently across several different longstanding technical fields. For surveys of some of these ideas (with a focus on numerical considerations), see e.g. Brogliato et al. (2002), Gilardi and Sharf (2002).

\footnotetext{
${ }^{3}$ An execution of a hybrid dynamical system exhibits the Zeno phenomena if it undergoes an infinite number of discrete or logical switches in finite time (Definition 6).
} 


\subsubsection{Numerical Simulation Methods}

While this paper is focused on a model for analysis and not simulation, it is informative to consider how other simulation strategies compare. The model developed here generates trajectories from the flow of hybrid dynamical systems defined by differential-algebraic equations (DAEs) between discrete transitions and so, in the language of (Brogliato et al. 2002, Sec. 6.3), simulations of these trajectories could be obtained ${ }^{4}$ via an event-driven scheme, as opposed to a penalized-constraint/continuous-contact scheme, or a time-stepping scheme.

Event-driven schemes have a long history, e.g. (Wehage and Haug 1982), (Pfeiffer and Glocker 1996), (Brogliato et al. 2002, Sec. 6.7), and include the hybrid dynamical systems formulations outlined in the next section. Typically they entail alternating between integration of smooth dynamics involving (usually) finite forces from contacts and the discontinuous handling of constraint addition or deletion (the "events"). Here, we extend these methods and codify the event-driven scheme in terms of a formal hybrid dynamical system. In contrast, some event-driven schemes formulate the contact dynamics as always consisting of impulses, e.g. Mirtich and Canny (1995). These impulsebased simulations combine both smooth and discontinuous contact interactions into impulses, with a continuous-time ballistic trajectory in between events.

Time-stepping schemes, which also account for contact interactions only using impulses by integrating applied forces over small time steps, are numerically efficient especially for systems with large numbers of constraints, see e.g. (Stewart and Trinkle 1996) (Anitescu and Potra 1997), or (Brogliato et al. 2002, Sec. 7). These models can be relaxed (by allowing contact forces and impulses to arise even before contact occurs) to enable efficient numerical simulation and motion synthesis (Drumwright and Shell 2011, Todorov et al. 2012). These methods allow contact constraints to be added or removed at any time step, but only once per time step. Furthermore, no distinction between continuous contact forces and discontinuous impulses is made. In this way these methods relax the requirements of the Principle of Constraints, i.e. that, "Constraints shall be maintained by forces, so long as this is possible; otherwise, and only otherwise, by impulses" (Kilmister and Reeve 1966, p. 79) (as noted e.g. in Stewart and Trinkle 1996, Section 1). Their advantage in avoiding many of the well explored physical paradoxes of rigid body mechanics (including Zeno phenomena (Drumwright 2010) as well as apparent contradictions between frictional forces and impulses discussed in Section 1.3.5) seems to come at the cost of persistence of contact. In contrast, here, persistence is one of the key simplifying modeling assumptions, expressing our intuitive experience of limbs interacting with the world, enabling some of the other assumptions, and affording our strong formal results. Despite being targeted at a different numerical integration scheme, many of the results in this paper, such as the consistent handling of massless limbs, are potentially applicable to time-stepping schemes.

\subsubsection{Hybrid Dynamical Systems}

This paper models manipulation and self-manipulation systems using a hybrid systems paradigm that assumes instantaneous transitions. Though we develop our (so-called) self-manipulation hybrid dynamical system for a similar class of mechanical systems as that considered in (van der Schaft and Schumacher 1998, Ex. 3.3), we specialize from the more general class of hybrid automata considered in (Lygeros et al. 2003, Def. II.1) to facilitate connections with the broader hybrid systems literature. Our self-manipulation system is closely related to the $n$-dimensional hybrid system of (Simic et al. 2005, Def. 2.1), the simple hybrid system of (Or and Ames 2011, Def. 1), and hybrid dynamical system of (Burden et al. 2015b, Def. 1) as we require: (i) multiple disjoint domains of varying dimension, disallowed by Or and Ames (2011), Simic et al. (2005); (ii) guards with arbitrary codimension, disallowed by Burden et al. (2015b); and we desire (iii) more analytical and geometric structure than is provided by the general framework in Lygeros et al. (2003), specifically domains that are differentiable manifolds and guards that are sub-analytic. Note that (i) is precluded in Or and Ames (2011), Simic et al. (2005) only for notational expediency since any multitude of domains may be embedded as disjoint submanifolds of a high-dimensional Euclidean space. The condition (ii) is excluded by Burden et al. (2015b) since it is generally incompatible with the results contained therein.

One property of hybrid systems that is crucial to establish for the present setting is that the guards are disjoint, i.e. no state is a member of two distinct guards, so there is no ambiguity as to which reset map to apply. This key property yields the proof that the model is deterministic (Lygeros et al. 2003, Def. III.2). Furthermore the system is set up such that every point on the boundary of the domain where the flow points outward is a member of a guard, thus guaranteeing that the system is non-blocking (Lygeros et al. 2003, Def. III.1), i.e. the execution continues for infinite time.

\footnotetext{
${ }^{4}$ E.g. using the algorithm proposed in Burden et al. (2015a) for hybrid control systems.
} 
The self-manipulation hybrid system developed in this paper uses the active contact constraints to define the discrete state or status (that we call the mode), as in e.g. Brogliato et al. (2002), Hurmuzlu and Marghitu (1994). However even when starting with a simple Lagrangian hybrid system without modes for every contact condition it appears to be useful to add such states to allow executions to be completed beyond a so-called "Zeno equilibrium" (Ames et al. 2006, Or and Ames 2011). Furthermore, the pseudo-impulse we introduce avoids certain Zeno executions by allowing the system to remain in a constrained mode after finitely many transitions, in a manner analogous to but formally distinct from the truncation proposed in Ames et al. (2006), Or and Ames (2011).

\subsubsection{Consistent Complementarity}

Any formulation that allows for persistent contact through impact must determine which contacts to make active and which to remove ${ }^{5}$. When there is no impulse (i.e., no constraint to add, but one or more constraints have violated the unilateral constraint cone), the removal process is called force-acceleration complementarity, as it is commonly modeled by a complementarity problem involving contact force and separating acceleration, e.g. (Trinkle et al. 1997, Eqn. 12), (Brogliato et al. 2002, Eqn. 10), where in the simplest case of a single contact point with zero or negative contact force it is simply removed. This complementarity problem framework can introduce paradoxical consequences in certain physical problem settings, for example in taking the rigid limit of a deformable body (Chatterjee 1999). It can also be computationally efficient to relax the hard constraints of the complementarity conditions, resulting in a convex optimization problem (Drumwright and Shell 2011, Todorov 2011).

An impulse induced from one or more contact constraints becoming active generally necessitates the removal of other constraints, specifically, those that require a negative impulse to remain. When invoked as a modeling principle, this impulse-velocity complementarity precludes a simultaneous impulse and separation velocity at a particular contact, e.g. (Lötstedt 1982, Eqn. 2.10b), (Brogliato et al. 2002, Eqn. 9). Imposing this modeling discipline affords the well established benefit of yielding a unique post-collision state for collisions modelled as plastic frictionless impacts (Cottle 1968, Heemels et al. 2000, Ingleton 1966, van der Schaft and Schumacher 1998). Unfortunately further generalizations can lead to inconsistencies and ambiguities (Chatterjee 1999, Hurmuzlu and Marghitu 1994, Ivanov 1995, Seghete and Murphey 2010). The existence and uniqueness of a solution must therefore be separately established in each physical circumstance that includes friction - or merely be assumed.

Massless legs introduce new problems into the complementarity problem. The massless leg condition in general, as introduced in (Johnson and Koditschek 2013a, Assumption C.6) and also used in countless prior works, e.g. (Blickhan 1989, Holmes et al. 2006, Kajita et al. 1992), allows for the neglect of certain states deemed inconsequential to the dynamics of interest when unconstrained (of course, the appropriateness of this neglect is task dependent rather than in any way intrinsic to the underlying physics, c.f. (Johnson and Koditschek 2013a, Sec. IV.C.5) or (Balasubramanian et al. 2008)). Indeed a massless leg that is not touching the ground is unconstrained and its position can be taken as arbitrary (or regarded as evolving according to dynamics sufficiently decoupled as to be considered independent), as used in the behavior analysis in (Johnson and Koditschek 2013a, Sec. IV.C.3). However the complementarity condition as used in e.g. (Lötstedt 1982, Eqn. 2.10b), (Brogliato et al. 2002, Eqn. 9), and listed in (51)-(52) is illposed in the absence of mass since there is no well-defined separation velocity or acceleration, nor anything precluding all massless contact points from always separating (at least for the dynamic model of interest here, as opposed to a quasistatic model, (Trinkle and Zeng 1995)). Instead here we reformulate the complementarity condition as (46)-(47) to not depend on the separation velocity.

\subsubsection{Impact Mechanics}

The usual Newtonian impact law (as in e.g. (Chatterjee and Ruina 1998, Eqn. 3), (Featherstone 2008, Eqn. 11.65) and many others) can be thought of as a mass-orthogonal projection onto the constraint manifold as used in e.g. augmented Lagrangian techniques (Bayo and Ledesma 1996, Eqn. 25). More generally, Moreau (1985) showed that impact problems can be modeled using measure differential inclusions. The algebraic plastic impact law involves inversion of the inertia tensor, which precludes the possibility of massless limbs and necessitates the reformulation given in this paper. Even if there are no truly massless links, a nearly massless body segment yields a poorly-conditioned inertia tensor (Johnson 2014, Sec. 5.1.1), leading to similar formulations as the one presented here in (Westervelt et al. 2003, Eqn. 9) or, for continuous time dynamics, (Holmes et al. 2006, Sec. 4.3) (Featherstone 2008, Eqn. 3.17).

\footnotetext{
${ }^{5}$ The removal ends up being the harder question, as "there is no problem in deciding when and which constraint to add to the active set since there is a constraint function to base the decision on. The problem of dropping constraints is more delicate...” (Lötstedt 1982, p. 283).
} 
In this paper we restrict our attention to systems modeled as exhibiting only perfectly plastic impact (perfectly inelastic impact). In the elastic impact case, it is necessary to consider the relative stiffness of contact points; depending on the restitution law invoked, multiple outcomes are consistent with the constitutive assumptions (Chatterjee and Ruina 1998, Hurmuzlu and Marghitu 1994). Though it is possible to bypass this technical obstacle by introducing an additional constitutive hypothesis, e.g. (Ballard 2000, H3 in Sec. 3.3), it remains to be validated (either theoretically or experimentally) that such assumptions accurately represent the physical system's behavior. Plastic impact avoids these inconsistencies, but more importantly we claim plastic impact provides a more useful model of the robotic systems of interest. Elastic impact is clearly needed in some robotics applications such as juggling (Buehler et al. 1994, Schaal and Atkeson 1993), tapping (Huang and Mason 1998) or ping-pong (Andersson 1989), but plastic impact, where there is no restitution and therefore no separation velocity after impact, is a more desirable model for most forms of locomotion (when it is important to keep feet on the ground) (Chatterjee et al. 2002, Westervelt et al. 2003) and manipulation (when it is important to keep fingers on the object) (Chatterjee et al. 2002, Wang and Mason 1987).

The new pseudo-impulse presented here, in addition to the Zeno results mentioned above, eliminates other evidently unwanted transitions by allowing the continuous-time forces to play a role in the impact process which is primarily "logical" (as opposed to energetic). This role may be best summarized by comparison to the most common alternatives. For example, instead of introducing a variable coefficient of restitution (Quinn 2005) (which our plastic impacts of interest already eliminate), the pseudo-impulse is not applied to the continuous (energetic) system directly but instead used to regularize the complementarity driven hybrid switching logic. Or as a second point of comparison, rather than introducing a fixed dead zone in impact energy (Pagilla and Yu 2001) or velocity (Brogliato et al. 2002, Sec. 6.4), the magnitude of the effect on our model's hybrid logic is not fixed but rather scales with the continuous time forces. An effect similar to this pseudo-impulse condition is also introduced by time-stepping simulations (Anitescu and Potra 1997, Stewart and Trinkle 1996), which, true to their name, always consider forces over small but finite time-steps. Under such schemes the magnitude of this effect is not a fixed, independent, user-imposed parameter since it must remain proportional to the duration of each time-step. Our preference for the independent, fixed choice reflects both mathematical convenience (the clearly defined hybrid dynamical system with its formal properties) as well as our taste in preferring to work with robotics models targeted for specific physical environments and settings.

\subsubsection{The Effect of Friction Models}

While this paper focuses on the impact problem, which friction greatly complicates (Keller 1986, McGeer and Palmer 1989, Trinkle et al. 1997, Wang and Mason 1987, Wang and Kumar 1994), even simulating continuous-time dynamics of rigid bodies with friction can be difficult (formally $N P$-hard (Baraff 1991)) due to the possibility of "jamming" events (Dupont and Yamajako 1994, Mason and Wang 1988), first attributed to Painlevé (1895). In this paper, following the model from Johnson and Koditschek (2013a), strong assumptions about frictional contact avoid these issues and enable integration of the dynamics as a differential-algebraic equation (DAE). As noted above, an alternative method to numerically solving these problems is the time-stepping approaches pursued in, e.g., Anitescu and Potra (1997), Stewart and Trinkle (1996), which resolve these issues by allowing for impulses at any time step. To resolve these issues in more general extensions of the system presented here (in particular those that are not well modeled by the frictional assumption, A12), the hybrid dynamical system could similarly be extended by allowing impulses at times without collisions, with such jamming events considered with additional guards and reset maps. We refer the interested reader to "Is Painlevé a real obstacle?" (Brogliato et al. 2002, Sec. 8.1) for further discussion of these issues.

\section{Modeling Assumptions}

The continuous Lagrangian dynamics of self-manipulation is specified in Johnson and Koditschek (2013a) using the notation and terminology of Murray et al. (1994) and summarized in Section 2.1. We continue to work within that framework here and briefly list the subtle differences between these two classes of systems in Section 2.2. However the impulsive dynamics (instantaneous changes in velocity when a new contact is added) were not specified in either, and so we introduce a plastic impact model in Section 2.5 and explore the induced complementarity conditions in Section 2.6. In addition, we make explicit how the massless leg (Section 2.3) and frictional assumptions (Section 2.8) made in Johnson and Koditschek (2013a) affect both the continuous time (Section 2.4) and impulsive dynamics, leading to a new formulation for the dynamics that is equivalent to the usual formulation when there are no massless links. Finally, Section 2.7 introduces a new pseudo-impulse that eliminates certain Zeno executions and related chattering behavior. 


\begin{tabular}{ll}
\hline \hline $\mathbf{a}: \mathcal{Q} \rightarrow \mathcal{C}$ & Base constraint function (2.1) \\
$\mathbf{A}: T \mathcal{Q} \rightarrow T \mathcal{C}$ & Velocity constraint function (2.1) \\
$\mathbf{A}^{\dagger}: T^{*} \mathcal{Q} \rightarrow T^{*} \mathcal{C}$ & Force constraint function (8) \\
$C^{r}, r \in \mathbb{N} \cup\{\infty, \omega\}$ & $C^{r}$ differentiable function (2.1) \\
CPPRED $: T \mathcal{Q} \rightarrow 2^{\mathcal{K}}$ & Solution to the PRED predicate (32) \\
$\overline{\mathbf{C}}: T \mathcal{Q}^{2} \rightarrow T^{*} \mathcal{Q}$ & Coriolis forces (12) \\
$\mathrm{FA}: 2^{\mathcal{K}} \times T \mathcal{Q} \rightarrow \mathbb{B}$ & Force-acceleration predicate (38) \\
$i, j, k \in \mathcal{K}$ & Contact constraints (2.1) \\
$I, J, K \subseteq \mathcal{K}$ & Set of active contact constraints (2.1) \\
$\mathcal{I} \subseteq \mathcal{K}$ & Complementarity scope (33) \\
$\mathbf{I d}, \mathbf{I} \mathbf{d}_{\mathbf{q}}$ & Identity matrix, of dimension $|\mathcal{Q}|(2.1)$ \\
$\mathrm{IV}: 2^{\mathcal{K}} \times T \mathcal{Q} \rightarrow \mathbb{B}$ & Impulse-velocity predicate (50) \\
$\mathcal{K}:=\mathcal{K}_{n} \cup \mathcal{K}_{t} \subset \mathbb{N}$ & Set of all contact constraints (2.1) \\
$\overline{\mathbf{M}}: T^{2} \mathcal{Q} \rightarrow T^{*} \mathcal{Q}$ & Inertia tensor (2.3) \\
$\overline{\mathbf{M}}{ }^{\dagger}: T^{*} \mathcal{Q} \rightarrow T^{2} \mathcal{Q}$ & Constrained inverse inertia tensor (8) \\
$\overline{\mathbf{N}}: \mathcal{Q} \rightarrow T^{*} \mathcal{Q}$ & Potential forces (e.g. gravity) (12) \\
$\mathrm{NTD}: T \mathcal{Q} \rightarrow \mathbb{B}$ & New touchdown predicate (22) \\
$\mathbf{P} \in T^{*} \mathcal{Q}$ & Impulse in state space (2.5) \\
$\widehat{\mathbf{P}}, \widetilde{\mathbf{P}} \in T^{*} \mathcal{C}$ & Impulses in constraint space (25), (56) \\
$\mathrm{PIV}: 2^{\mathcal{K}} \times T \mathcal{Q} \rightarrow \mathbb{B}$ & Pseudo-impulse IV predicate (58) \\
$\mathbf{q} \in \mathcal{Q}:=\Theta \times S E(\mathrm{~d})$ & Continuous state (2.1) \\
$\mathbf{T q}:=(\mathbf{q}, \dot{\mathbf{q}}) \in T \mathcal{Q}$ & Continuous state and velocity (2.1) \\
$\mathrm{TD}: \mathcal{K}{ }_{n} \times T \mathcal{Q} \rightarrow \mathbb{B}$ & Touchdown predicate (21) \\
$\mathbf{U}: T^{*} \mathcal{C} \rightarrow \mathbb{R}|\mathcal{C}|$ & Unilateral constraint cone (2.1) \\
$\alpha: \mathcal{K} \rightarrow \mathcal{K} n$ & Corresponding normal constraint (2) \\
$\delta_{t} \in \mathbb{R}^{+}$ & Small time duration of impact (56) \\
$\Delta \dot{\mathbf{q}} \in T \mathcal{Q}$ & Instantaneous change in velocity (2.5) \\
$\lambda \in T^{*} \mathcal{C}$ & Lagrange multipliers (13) \\
$\Lambda: T^{2} \mathcal{C} \rightarrow T^{*} \mathcal{C}$ & Constrained contact inertia tensor (8) \\
$\Upsilon \in T^{*} \mathcal{Q}$ & External forces and torques (12) \\
$\prec, \succ, \preceq, \succeq, \equiv$ & Trending negative/positive (Def. 1) \\
\hline
\end{tabular}

Table 1: Key symbols used throughout this paper, with section or equation number of introduction marked. See also Table 2 for symbols introduced in Section 3.

\subsection{Setup and Notation}

The notation used in this paper is chosen to be consistent with (Johnson and Koditschek 2013a, Table I) (and agreeing where possible with Murray et al. (1994)) or is defined as it is used and summarized in Table 1. The base component of the state is denoted, $\mathbf{q} \in \mathcal{Q}$, while the full state is, $\mathbf{T q}:=(\mathbf{q}, \dot{\mathbf{q}})$, and this state completely describes the motion of interest, as,

Assumption A1 (Rigid Bodies). The robot is made up of a finite number of rigid bodies whose configuration lies in a connected complete $C^{\omega}$ Riemannian manifold $\mathcal{Q}$.

Since the configuration spaces of many extant robots are not linear (e.g., due to rotary joints, rigid body rotations, or constrained mechanisms), it is most natural to invoke the general framework of differentiable manifolds to model the state space. For concreteness we consider the case where $\mathcal{Q}:=\Theta \times S E(\mathrm{~d})$ consists of joint angles and the special Euclidean group of dimension d, but our formal results are stated for an arbitrary connected complete $C^{\omega}$ Riemannian manifold $\mathcal{Q}$. We recognize that this generality necessitates mathematical formalisms and notation that are not uniformly adopted in the robotics community (exceptions such as Murray et al. (1994) notwithstanding); we aim whenever possible to translate unfamiliar objects into standard terminology and provide a terse overview of the background material needed to parse the more general case in Appendix C. 
We are concerned with sets of contact constraints (e.g., $I, J, K \subset \mathcal{K}$ ) that we shall call modes or contact modes hereinafter, subsets of indices whose particular elements (e.g., $i, j, k \in \mathcal{K}$ ) index the contact constraints that prevail at some instant (Johnson and Koditschek 2013a, Sec. II.C) (Murray et al. 1994, Sec. 5.2.1). In addition to contact with the robot's environment, contact constraints may include cases of self-contact as well as joint limits. The universe of all possible constraint indices from which these subsets are taken is denoted $\mathcal{K}=\mathcal{K}_{n} \cup \mathcal{K}_{t}$, partitioned by those that are in the normal (non-penetrating) direction and those that are in tangential (non-sliding) direction. Similarly, for any set of constraints specified by mode $I$, define the subsets $I_{n}:=I \cap \mathcal{K}_{n}$ and $I_{t}:=I \cap \mathcal{K}_{t}$, where clearly $I=I_{n} \cup I_{t}$ and $I_{n} \cap I_{t}=\varnothing$.

Contact constraints in the normal direction ${ }^{6}, i \in \mathcal{K}_{n}$, specify a holonomic constraint of the form $\{(\mathbf{q}, \dot{\mathbf{q}}) \in T \mathcal{Q}$ : $\left.\mathbf{a}_{i}(\mathbf{q})=0\right\}$ for $\mathbf{a}_{i}: \mathcal{Q} \rightarrow \mathbb{R}$ (and whose corresponding velocity constraint $\mathbf{A}_{i}: T \mathcal{Q} \rightarrow \mathbb{R}$ is equivalent to the Jacobian $D \mathbf{a}_{i}$, (Johnson and Koditschek 2013a, Eqn. 11)), while those in the tangential direction, $i \in \mathcal{K}_{t}$, specify a nonholonomic constraint of the form $\left\{(\mathbf{q}, \dot{\mathbf{q}}) \in T \mathcal{Q}: \mathbf{A}_{i}(\mathbf{q}) \dot{\mathbf{q}}=0\right\}$ where again $\mathbf{A}_{i}: T \mathcal{Q} \rightarrow \mathbb{R}$. For a given contact mode $I$, the space of constrained positions is a manifold $\mathcal{C}_{I}$ of dimension $|I|$ (i.e., the number of constraints in $I$ ).

In the interest of notational clarity, we generally express functional dependence on contact modes via subscript, e.g., $X_{I}(\mathbf{q}, \ldots):=X(I, \mathbf{q}, \ldots)$, and when it is clear from context, we further suppress the subscript, e.g. $X(\mathbf{q}, \ldots)$. For example, and used extensively throughout this paper, fixing an ordering on $\mathcal{K}$ we obtain the velocity constraints active in mode $I, \mathbf{A}_{I}: T \mathcal{Q} \rightarrow T \mathcal{C}_{I}$, as a selection of rows from the set of all velocity constraints $\mathbf{A}_{\mathcal{K}}$, i.e., ${ }^{7}$

$$
\mathbf{A}_{I}(\mathbf{q}):=\mathbf{A}(I, \mathbf{q})=\pi_{I} \mathbf{A}_{\mathcal{K}}(\mathbf{q})
$$

where $\pi_{I}$ is the Boolean projection matrix formed by the rows of canonical unit vectors associated with the elements in the index set $I$. Similarly for a single constraint $i, \mathbf{A}_{i}:=\pi_{i} \mathbf{A}_{\mathcal{K}}=\mathbf{A}_{\{i\}}$.

We make the following assumption on the combined maps,

Assumption A2 (Simple Constraints). All constraints are independent, that is for all contact modes I, the maps $\mathbf{a}_{I_{n}}: \mathcal{Q} \rightarrow \mathcal{C}_{I_{n}}$ and $\mathbf{A}_{I}: T \mathcal{Q} \rightarrow T \mathcal{C}_{I}$ are constant rank.

We refer the reader to Appendix $\mathrm{C}$ for the definition of rank of a $C^{r}$ map; in coordinates, this condition states that the gradient vectors of each coordinate of the respective maps are linearly independent at every point. If this condition failed to hold, the configuration space could possess singularities that could preclude existence and/or uniqueness of trajectories for the mechanical system. Note that this precludes the possibility of redundant constraints, though there are methods of resolving such redundancies, e.g. in Greenfield et al. (2005). In particular, this requirement is met if $\mathbf{a}_{\mathcal{K}_{n}} \in C^{\omega}\left(\mathcal{Q}, \mathbb{R}^{\left|\mathcal{K}_{n}\right|}\right)$ and $\mathbf{A}_{\mathcal{K}} \in C^{\omega}\left(T \mathcal{Q}, \mathbb{R}^{|\mathcal{K}|}\right)$ are constant $\operatorname{rank}^{8}$.

We note that there is an assignment,

$$
\alpha: \mathcal{K} \rightarrow \mathcal{K}_{n},\left.\quad \alpha\right|_{\mathcal{K}_{n}}=\mathbf{I d},
$$

of contacts to normal contacts such that $\left.\alpha\right|_{\mathcal{K}_{t}}$ maps tangential contacts to the corresponding normal contact (where Id is the appropriate identity matrix). Note that for each $k \in \mathcal{K}_{t}$ and $j=\alpha(k), \mathbf{A}_{k}$ is orthogonal to $\mathbf{A}_{j}$.

It is well established that the motion of mutually constrained rigid bodies can be effectively modeled using polynomial maps (Wampler and Sommese 2013), hence imposing contact constraints arising from their interaction with the piecewise polynomial representations of the environment (commonly adopted by the sensory community (Lalonde et al. 2007)) leads to,

Assumption A3 (Analytic Constraints). All constraints are analytic functions, that is for all contact modes I, the maps $\mathbf{a}_{I_{n}}: \mathcal{Q} \rightarrow \mathcal{C}_{I_{n}}$ and $\mathbf{A}_{I}: T \mathcal{Q} \rightarrow T \mathcal{C}_{I}$ are $C^{\omega}$.

Given an analytic vector field subject to an analytic constraint, as shown in Lemma 3 it is possible to determine whether the constraint remains active over a nonzero time horizon by evaluating Lie derivatives at a single instant in time. If either the vector field or constraint were merely smooth, the differential equation determined by the vector field would, in general, need to be solved over a nonzero time horizon to determine whether the constraint remained active.

\footnotetext{
${ }^{6}$ Note that normal direction constraints for non-adhesive contact is unilateral, although within a contact mode they can be considered bilateral until the constraint force is violated (e.g. Lötstedt 1982, Sec. 4).

${ }^{7}$ However, note that most functions of the mode are not a simple projections, and so e.g. $\mathbf{A}_{I}^{\dagger}$, defined in (8), $\mathbf{A}_{I}^{\dagger} \neq \pi_{I} \mathbf{A}_{\mathcal{K}}^{\dagger}$, but rather $\mathbf{A}_{I}^{\dagger}$ is as defined in (8), i.e. constructed with the corresponding $\mathbf{A}_{I}$.

${ }^{8}$ This stronger assumption would not be true if there were two parallel constraints that, due to geometry, could not simultaneously be active, in which case the original requirement must be checked for all $I$.
} 
Assumption A4 (Persistent Contact). Contact with the world occurs through a finite number of active constraints indexed by $I \subset \mathcal{K}$ that apply continuous time forces. Furthermore, contact persists until the next event (e.g. touchdown or liftoff).

This assumption is related to the Principle of Constraints, as discussed in Section 1.3.1. Its adoption partitions trajectories so that at all times between instantaneous touchdown or liftoff events there persists a well-defined set of active constraints (enabling the systematic a priori enumeration and analysis of these constraint sets and their sequences, e.g. Johnson and Koditschek (2013b)). This contrasts with simulations generated by time-stepping algorithms, wherein contact Stewart and Trinkle (1996) or interpenetration Anitescu and Potra (1997) are resolved only at multiples of the timestep, and no distinction between forces and impulse are made (indeed this relaxation is what enables the efficient and consistent simulation in such formulations).

The impact problem can be summarized as determining which constraints to add or remove from the active set. The active set continues to constrain the system so long as the unilateral constraint cone (Johnson and Koditschek 2013a, Eqn. 7) is positive, $\mathbf{U}(\boldsymbol{\lambda}) \geq 0$, where $\lambda \in T^{*} \mathcal{C}$ is the vector of Lagrange multipliers (constraint forces) (Johnson and Koditschek 2013a, Eqn. 33). Included in $\mathbf{U}$ is both the non-attachment condition that normal direction forces are positive as well as the friction cone that relates the magnitude of the normal and tangential components.

In the complementarity problems, the following definition simplifies statements involving higher-order derivatives of the state that seem to arise unavoidably (as stated in (van der Schaft and Schumacher 1998, Sec. 3), (Heemels et al. 2000, Sec. 1), formalizing the concepts represented in e.g. (Featherstone 2008, Fig. 11.4), (Siciliano and Khatib 2008, Sec. 27.2)),

Definition 1. Given a smooth function $h: M \rightarrow \mathbb{R}$ defined over a smooth manifold $M$, a point $x \in M$, and a smooth vector field $F: M \rightarrow T M$, we say that $h$ is trending negative with respect to the vector field $F$ at $x$, denoted $h(x) \prec_{F} 0$, (or $h(x) \prec 0$ if the context specifies $F$ ), if,

$$
\exists m \geq 0:\left(\mathcal{L}_{F}^{m} h\right)(x)<0 \wedge \forall \ell<m:\left(\mathcal{L}_{F}^{\ell} h\right)(x)=0,
$$

where $\mathcal{L}_{F}^{m} h: M \rightarrow \mathbb{R}$ is the $m^{\text {th }}$ Lie derivative $e^{9}$ of $h$ with respect to the vector field $F$. Similarly, we say that $h$ is trending positive at $x$, denoted $h(x) \succ 0$, when $-h(x) \prec 0$. We say that $h$ is identically zero at $x$, denoted $h(x) \equiv 0$, when $\forall \ell \in \mathbb{N}:\left(\mathcal{L}_{F}^{\ell} h\right)(x)=0$. Finally, we say that $h$ is trending non-negative at $x$, denoted $h(x) \succeq 0$, when $h(x) \succ 0$ or $h(x) \equiv 0$, and that $h$ is trending non-positive at $x$, denoted $h(x) \preceq 0$, when $h(x) \prec 0$ or $h(x) \equiv 0$.

We refer the reader to Appendix $\mathrm{C}$ for the definition of a vector field $F: M \rightarrow T M$; in the case where $M=\mathbb{R}^{n}$, the tangent bundle $T M$ can be canonically identified with $\mathbb{R}^{n}$ to obtain a more familiar function $\widetilde{F}: \mathbb{R}^{n} \rightarrow \mathbb{R}^{n}$ that determines an ordinary differential equation $\dot{\widetilde{x}}=\widetilde{F}(\widetilde{x})$.

That is, $h(x) \prec 0$ if and only if the following vector,

$$
\left[h(x),\left(\mathcal{L}_{F} h\right)(x),\left(\mathcal{L}_{F}^{2} h\right)(x), \ldots\right],
$$

is lexicographically smaller than zero (Bertsimas and Tsitsiklis 1997, Def. 3.5). As an example of when these properties are important, consider the examples in Figure 2. In each case, the initial configuration (taken as the bottom point of the circle) is $\mathbf{q}=\left[\begin{array}{ll}x y & y\end{array}\right]^{T}=\left[\begin{array}{ll}0 & 0\end{array}\right]^{T}$, and the initial velocity is $\dot{\mathbf{q}}=\left[\begin{array}{ll}v & 0\end{array}\right]^{T}, v>0$. Assume that the particle has unit mass $\left(\overline{\mathbf{M}}=\mathbf{I} \mathbf{d}_{2}\right)$, and that there are no non-contact forces $(\overline{\mathbf{N}}=\mathbf{0}, \overline{\mathbf{C}}=\mathbf{0}, \Upsilon=\mathbf{0}$, as defined in Section 2.4). In all cases the particle is touching the constraint $(\mathbf{a}(\mathbf{q})=0)$ but has no impacting or separating velocity $(\mathbf{A} \dot{\mathbf{q}}=0)$, so there is no impulse (as defined in Section 2.5). Furthermore in c) and d) there is no impacting or separating acceleration (A $\ddot{\mathbf{q}}+\dot{\mathbf{A}} \dot{\mathbf{q}}=0)$. However in a) and c) the constraint function is trending positive, $\mathbf{a}(\mathbf{q}) \succ 0$, while in b) and d) the constraint function is trending negative $\mathbf{a}(\mathbf{q}) \prec 0$.

Furthermore, we make use of the following properties of this trending relation,

Lemma 1. The closure of $\{x: h(x) \prec 0\}$ or $\{x: h(x) \preceq 0\}$ is $\{x: h(x) \leq 0\}$, while the closure of $\{x: h(x) \succ 0\}$ or $\{x: h(x) \succeq 0\}$ is $\{x: h(x) \geq 0\}$.

This is easy to see as $\{x: h(x)<0\} \subset\{x: h(x) \preceq 0\} \subset\{x: h(x) \leq 0\}$ for any vector field.

\footnotetext{
${ }^{9}$ See e.g. (Lee 2012, Ch. 9), and note our convention that, $\mathcal{L}_{F}^{0} h=h$.
} 


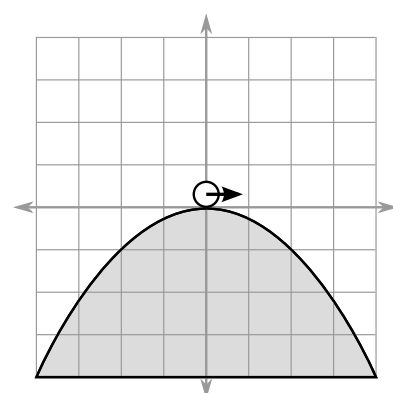

(a)

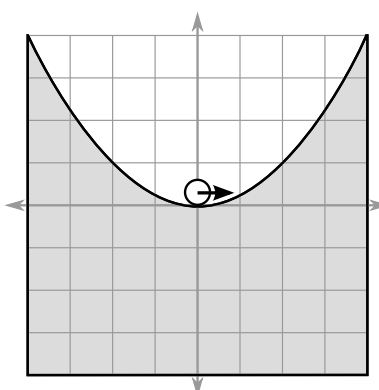

(b)

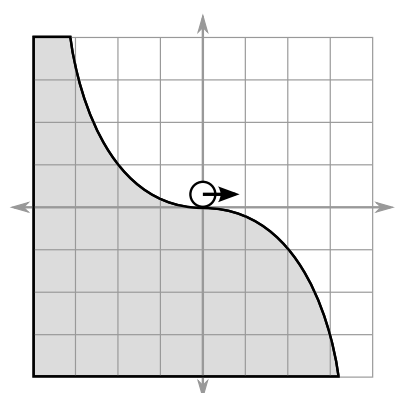

(c)

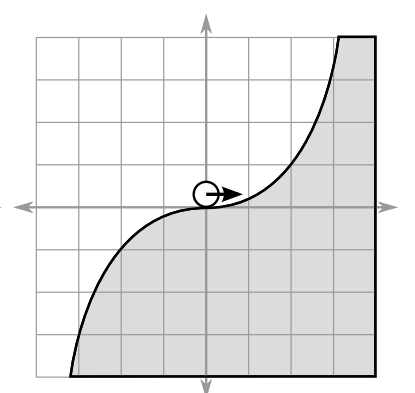

(d)

Figure 2: Four examples of a planar point particle $\left(\mathcal{Q}=\mathbb{R}^{2}\right)$ with a single constraint $(\mathcal{K}=\{1\})$, defined as (a) $\mathbf{a}=$ $x^{2}+4 y$, (b) $\mathbf{a}=-x^{2}+4 y$, (c) $\mathbf{a}=x^{3}+8 y$, and (d) $\mathbf{a}=-x^{3}+8 y$. Note that if the particle velocity is directed to the right $\left(\dot{\mathbf{q}}=\left[\begin{array}{ll}v & 0\end{array}\right]^{T}, v>0\right)$, as illustrated, then: the constraint function is trending positive $(\mathbf{a}(\mathbf{q}) \succ 0)$ in (a) and (c); the constraint function is trending negative $(\mathbf{a}(\mathbf{q}) \prec 0)$ in $(b)$ and $(\mathrm{d})$.

Lemma 2. Given a smooth vector field, $F: M \rightarrow T M$, a point in a smooth boundaryless manifold, $x \in M$, and a smooth positive function, $g: M \rightarrow \mathbb{R}^{+}$, any other smooth function, $h: M \rightarrow \mathbb{R}$, is trending negative if and only if its product with $g$ has the same property, i.e.,

$$
h(x) \prec_{F} 0 \Leftrightarrow g(x) \cdot h(x) \prec_{F} 0 .
$$

See Appendix A for a proof.

Lemma 3. Let $h: M \rightarrow \mathbb{R}$ be a $C^{\omega}$ function and $F: M \rightarrow T M$ be a $C^{\omega}$ vector field over a $C^{\omega}$ boundaryless manifold $M$, and let $\chi:(-\varepsilon,+\varepsilon) \rightarrow M$ denote an integral curve for $F$ through $x:=\chi(0)$. Then $h$ is trending positive at $x$ with respect to $F, h(x) \succ_{F} 0$, if and only if there exists $\delta \in(0, \varepsilon)$ such that,

$$
\forall s \in(0,+\delta): h \circ \chi(s)>0 .
$$

The requirement that the manifold be boundaryless is introduced to simplify the statement of this Lemma; the Lemma clearly applies to the interior of a manifold with corners (which is, after all, simply a manifold without boundary) (Joyce 2012, Def. 2.1).

To see that the lemma is true, note that if $\chi$ is an integral curve for $F$ such that $h \circ \chi(s)$ is positive for $s>0$ sufficiently small, then since $h$ is analytic we conclude (3) is satisfied. The other direction follows easily by contradiction using the mean value Theorem. We note that this is not true if $h$ or $F$ are merely $C^{\infty}$. Also note that the conditions of the lemma do not imply that $\forall s \in(-\delta, 0): h \circ \chi(s)<0$ for two reasons: 1) it is possible that $h(x) \neq 0$, and 2) even for $h(x)=0$, grazing contact would handled incorrectly, as in Figure 2, example (a).

Lemma 3 implies a computationally efficient way to test these trending conditions is to simply integrate a flow until it reaches a zero crossing, as discussed further in Section 4.4.

\subsection{Manipulation and Self-Manipulation}

This section briefly summarizes the self-manipulation formalism introduced in Johnson and Koditschek (2013a), as it relates to manipulation, e.g. as presented in Murray et al. (1994). Each defines a number of frames on the robot and its environment - the palm frame, the object frame, the contact frame, etc. In an effort to keep the problems as similar as possible, the following conventions were adopted in Johnson and Koditschek (2013a),

- In self-manipulation, the robot is the object being manipulated and so to properly consider the forces and torques on the object the robot's palm frame, $P$, and the object frame, $O$, are chosen to be coincident, (Johnson and Koditschek 2013a, Sec. II-B).

- Thus motions that, in a manipulation problem, move an object to the right really move the robot to the left, and so the self-manipulation grasp map (a component of $\mathbf{A}$ ) is a reflection of the manipulation grasp map, $\mathbf{G}_{s}:=-\mathbf{G}$, (Johnson and Koditschek 2013a, Eqn. 15). 
- By convention the contact frame is defined at any point of contact with the $z$-axis pointing into the object (away from the finger tip), (Murray et al. 1994, Sec. 5.2.1). In self-manipulation the convention of (Johnson and Koditschek 2013a, Sec. II-C) is to keep the contact frame consistent with respect to the legs, and so the $z$-axis points away from the robot and into the ground. This results in a unilateral constraint cone, $\mathbf{U}$, that is negative, (Johnson and Koditschek 2013a, Eqn. 76, 78).

- Since the palm reference frame is accelerating with respect to the world, the inertia tensor, $\overline{\mathbf{M}}$, (Johnson and Koditschek 2013a, Eqn. 26), and by extension the Coriolis terms, $\overline{\mathbf{C}}$, (Johnson and Koditschek 2013a, Eqn. 30), are more coupled and lack the block diagonal structure present in manipulation problems, (Murray et al. 1994, Eqn. 6.24).

It should be no surprise that the problem formulations are structurally equivalent since the underlying kinematics and dynamics are indifferent to the problem category. However owing to the notational differences summarized above, through the remainder of this paper we choose to write out the problems in terms of a self-manipulation system, with the understanding that the results contained herein apply equally well to manipulation systems once these transformations are incorporated.

\subsection{Massless Considerations}

To properly define the dynamics of a partially massless system, consider a parametrized family of singular semiRiemannian metrics,

$$
\overline{\mathbf{M}}_{\mathcal{\varepsilon}}(\mathbf{q}): \mathcal{Q} \times[0, \bar{\varepsilon}] \rightarrow \mathbb{R}^{\mathrm{q} \times \mathrm{q}},
$$

such that $\overline{\mathbf{M}}_{0}(\mathbf{q}):=\overline{\mathbf{M}}$ is the (possibly) degenerate inertia tensor for the system (Johnson and Koditschek 2013a, Eqn. 26) and may be singular, while $\varepsilon$ assigns a small mass and inertia to any putatively massless links such that $\overline{\mathbf{M}}_{\varepsilon}(\mathbf{q})$ is full-rank for all $\varepsilon>0$ (for our present purposes, it is sufficient to use a limiting model such as $\overline{\mathbf{M}}_{\varepsilon}:=\overline{\mathbf{M}}_{0}+\varepsilon \mathbf{I} \mathbf{d}_{\mathrm{q}}$ rather than some more specific physically motivated one). We invoke the general definition of Riemmanian metric here since it provides the coordinate-invariant formulation of the familiar mass or inertia matrix associated with a collection of rigid bodies, and refer the reader to Lee (1997) for a formal definition and Section 2.4 for additional details. The dynamics of the system in contact mode $I$ can be expressed (as shown below) using the inverse of the following block matrix containing $\overline{\mathbf{M}}_{\mathcal{\varepsilon}}$, defining ${ }^{10} \mathbf{A}^{\dagger}: T^{*} \mathcal{Q} \rightarrow T^{*} \mathcal{C}, \overline{\mathbf{M}}^{\dagger}: T^{*} \mathcal{Q} \rightarrow T^{2} \mathcal{Q}$, and $\Lambda: T^{2} \mathcal{C} \rightarrow T^{*} \mathcal{C}$ as,

$$
\begin{aligned}
& {\left[\begin{array}{ll}
\overline{\mathbf{M}}_{I}^{\dagger} & \mathbf{A}_{I}^{\dagger T} \\
\mathbf{A}_{I}^{\dagger} & \Lambda_{I}
\end{array}\right]:=\lim _{\varepsilon->0}\left(\left[\begin{array}{cc}
\overline{\mathbf{M}}_{\varepsilon} & \mathbf{A}_{I}^{T} \\
\mathbf{A}_{I} & 0
\end{array}\right]\right)^{-1}} \\
& \quad=\left(\lim _{\varepsilon->0}\left[\begin{array}{rr}
\overline{\mathbf{M}}_{\varepsilon} & \mathbf{A}_{I}^{T} \\
\mathbf{A}_{I} & 0
\end{array}\right]\right)^{-1}=\left[\begin{array}{cc}
\overline{\mathbf{M}}_{0} & \mathbf{A}_{I}^{T} \\
\mathbf{A}_{I} & 0
\end{array}\right]^{-1} .
\end{aligned}
$$

From this definition, note that the following properties hold,

$$
\begin{aligned}
\mathbf{A}^{\dagger} \mathbf{A}^{T}=\mathbf{A} \mathbf{A}^{\dagger T} & =\mathbf{I d}, & \overline{\mathbf{M}}^{\dagger} \mathbf{A}^{T}=\mathbf{A} \overline{\mathbf{M}}^{\dagger} & =0, \\
\overline{\mathbf{M}}^{\dagger} \overline{\mathbf{M}}+\mathbf{A}^{\dagger T} \mathbf{A} & =\mathbf{I d}, & \mathbf{A}^{\dagger} \overline{\mathbf{M}}+\Lambda \mathbf{A} & =0 .
\end{aligned}
$$

To ensure that the inverse of the matrix in (9) (sometimes called the "Lagrangian matrix of coefficients", e.g. (Papalambros and Wilde 2000, Eqn. 7.79), and sometimes used in robotics for numerical reasons, e.g. (Holmes et al. 2006, Sec. 4.3)) is well-defined, we require some modeling assumptions on the nature of the massless appendages. Thus if the inverse exists, this $\varepsilon$-parametrized curve takes its image in $G L(n)$ (the group of invertible matrices over $\mathbb{R}^{n}$ ) within which matrix inversion is a continuous operation, hence the limit commutes with the inverse operation, and $\overline{\mathbf{M}}_{\varepsilon}^{\dagger}$ is a well defined smooth curve defined over all $\varepsilon \in[0, \bar{\varepsilon}]$.

To meet this requirement, massless appendages are allowed here only in a limited form,

\footnotetext{
${ }^{10}$ Note that (Johnson and Koditschek 2013a, Eqn. 40) used the notation $\mathbf{A}^{*}$ while in this paper we use $\mathbf{A}^{\dagger}$ to signify the slight difference in definition used here, and to avoid confusion with the pullback of $\mathbf{A}$, usually noted as $\mathbf{A}^{*}$, but which happens to be $\mathbf{A}^{\dagger T}$.
} 
Assumption A5 (Constrained Massless Limbs). For all limbs in contact with the world, any rank deficiencies of the inertia tensor $\overline{\mathbf{M}}$ (Johnson and Koditschek 2013a, Eqn. 26) are "corrected" by velocity constraints $\mathbf{A}$ sufficient to guarantee that any remaining allowed physical movement excites some associated kinetic energy, that is, the block matrix in (9) is invertible.

If the "rank correction" condition in this assumption were violated, then it would not be possible in general to determine the system's instantaneous acceleration solely from the internal, applied, and Coriolis forces; it could happen that either no accelerations are consistent with the net forces, or an infinite set of accelerations are. This condition admits its most physically straightforward expression via the requirement that the inertia tensor is nonsingular when written with respect to generalized or reduced coordinates, $\widetilde{\mathbf{M}}$ (i.e., any local chart arising from an implicit function solution to the constraint equation (Johnson and Koditschek 2013a, Eqn. 10)). However, for purposes of this paper, we find it more useful to work with the Lagrange-d'Alembert formulation of the constrained dynamics, (Johnson and Koditschek 2013a, Eqn. 33), hence, we translate that natural assumption into more formal algebraic terms governing the relationship between the lifted (velocity) constraints, A (Johnson and Koditschek 2013a, Eqn. 11), and the overall inertia tensor $\overline{\mathbf{M}}$ as follows,

Lemma 4. The matrix $\left[\begin{array}{cc}\overline{\mathbf{M}} & \mathbf{A}^{T} \\ \mathbf{A} & 0\end{array}\right]$, (9), is invertible if and only if the inertia tensor expressed in generalized or reduced coordinates, $\widetilde{\mathbf{M}}$ (Johnson and Koditschek 2013a, Eqn. 36), is invertible (Johnson and Koditschek 2013a, Sec. II.K, Assumption A.4).

as shown in Appendix B.1. See Section 4.2 for a discussion of physical scenarios that meet this requirement.

When not constrained on the ground, any such massless links or limbs must then be removed from consideration as mechanical degrees-of-freedom: since they are massless, when unconstrained, the associated joints can be considered to have arbitrary configuration. Their evolution is instead treated according to the principle,

Assumption A6 (Unconstrained Massless Limbs). For all limbs not in contact with the world, any components of the state that do not excite some kinetic energy must be removed from the usual dynamics and instead considered to evolve in isolation according to some independent, decoupled dynamics. ${ }^{11}$

In the same vein as the remark following Assumption A5 (Constrained Massless Limbs), we observe that it is not possible to uniquely determine accelerations of unconstrained massless limbs due to corresponding degeneracy in the inertia tensor. Excluding such limb states from the coupled Lagrangian mechanics governing the remaining body and limb segments enables us in the sequel to specify a differential-algebraic equation that admits unique solutions. As the dynamics of the excluded states do not affect those of the remaining states, for the rest of this section we abuse notation and suppress the subscript $I$ from the state space $\mathcal{Q}$, so that unless stated otherwise we are concerned with only the "active" component $\mathcal{Q}_{I}$ of the decomposed state space for the mode of interest. See also Section 4.5 for a discussion of Zeno (Def. 6) considerations with massless legs.

\subsection{Continuous Dynamics}

With this notation, the continuous-time dynamics of (Johnson and Koditschek 2013a, Eqn. 33) in contact mode I can be expressed as,

$$
\begin{aligned}
& \ddot{\mathbf{q}}_{I}:=\overline{\mathbf{M}}_{I}^{\dagger}\left(\Upsilon_{I}-\overline{\mathbf{C}}_{I} \dot{\mathbf{q}}-\overline{\mathbf{N}}_{I}\right)-\mathbf{A}_{I}^{\dagger T} \dot{\mathbf{A}}_{I} \dot{\mathbf{q}}, \\
& \lambda_{I}:=\mathbf{A}_{I}^{\dagger}\left(\Upsilon_{I}-\overline{\mathbf{C}}_{I} \dot{\mathbf{q}}-\overline{\mathbf{N}}_{I}\right)-\Lambda_{I} \dot{\mathbf{A}}_{I} \dot{\mathbf{q}},
\end{aligned}
$$

where $\Upsilon_{I}$ is the applied forces, $\overline{\mathbf{C}}_{I}$ is the centripetal and Coriolis forces, and $\overline{\mathbf{N}}_{I}$ is the nonlinear and gravitational forces (Johnson and Koditschek 2013a, Eqn. 30, 31).

\footnotetext{
${ }^{11}$ That is, in contact mode $I$, the configuration manifold $\mathcal{Q}$ decomposes as a product of manifolds $\mathcal{Q}=\mathcal{Q}_{I} \times \widetilde{\mathcal{Q}}_{I}$, where $\mathcal{Q}_{I}$ corresponds to a subset of the system coordinates such that the matrix in (9) is nonsingular, and $\widetilde{\mathcal{Q}}_{I}$ corresponds to the remaining coordinates. The dynamics for the coordinates of $\widetilde{\mathcal{Q}}_{I}$ is given by some vector field $\widetilde{F}_{I}$. Here we have written the dynamics as a second order vector field so that the dynamics of the full system may be written in a notationally consistent manner. This is not required; regardless of how the dynamics are defined for these coordinates, there is no coupling of energy with the rest of the system through the inertia tensor.
} 
When $\overline{\mathbf{M}}_{\varepsilon},(7)$, is invertible (including, possibly, even for $\varepsilon=0$ ), it is easy to verify the equivalences (and dropping for now the subscripted contact mode $I$ ),

$$
\begin{aligned}
\overline{\mathbf{M}}^{\dagger} & =\overline{\mathbf{M}}^{-1}-\overline{\mathbf{M}}^{-1} \mathbf{A}^{T}\left(\mathbf{A} \overline{\mathbf{M}}^{-1} \mathbf{A}^{T}\right)^{-1} \mathbf{A} \overline{\mathbf{M}}^{-1}, \\
\mathbf{A}^{\dagger T} & =\overline{\mathbf{M}}^{-1} \mathbf{A}^{T}\left(\mathbf{A} \overline{\mathbf{M}}^{-1} \mathbf{A}^{T}\right)^{-1} \\
\Lambda & =-\left(\mathbf{A} \overline{\mathbf{M}}^{-1} \mathbf{A}^{T}\right)^{-1}
\end{aligned}
$$

as shown in Appendix B, (114). Note that constructions such as these are commonly used in robotics when $\overline{\mathbf{M}}$ is invertible, e.g. (Khatib 1983, Eqns. 45-46) and many others (where their $\Lambda_{r}$ has the opposite sign of our $\Lambda$ and their $\bar{J}$ corresponds to $\mathbf{A}^{\dagger T}$, although note that the definition (15) is exact and not defined as a minimal-energy pseudo-inverse).

Lemma 5. When $\overline{\mathbf{M}}_{0}=\overline{\mathbf{M}}$ is invertible, the dynamics (12) and (13) are equivalent to the more common expression (as stated e.g. in the last equations of (Johnson and Koditschek 2013a, Appendix D), or (Murray et al. 1994, Eqn. 6.5, 6.6)),

$$
\begin{aligned}
& \ddot{\mathbf{q}}=\overline{\mathbf{M}}^{-1}\left(\Upsilon-\overline{\mathbf{C}} \dot{\mathbf{q}}-\overline{\mathbf{N}}-\mathbf{A}^{T} \lambda\right) \\
& \lambda=\left(\mathbf{A} \overline{\mathbf{M}}^{-1} \mathbf{A}^{T}\right)^{-1}\left(\mathbf{A} \overline{\mathbf{M}}^{-1}(\Upsilon-\overline{\mathbf{C}} \dot{\mathbf{q}}-\overline{\mathbf{N}})+\dot{\mathbf{A}} \dot{\mathbf{q}}\right) .
\end{aligned}
$$

The claim follows directly from substituting (14)-(16), the explicit solution to (8) when $\overline{\mathbf{M}}$ is invertible, into (12)(13), as worked out in Appendix B.2.

Whether $\overline{\mathbf{M}}$ is invertible or not, we require,

Assumption A7 (Lagrangian Dynamics). In each contact mode I, the time evolution of the active coordinates of the system are governed by Lagrangian dynamics, and the applied forces are such that the vector field defined by (12) for coordinates in $\mathcal{Q}_{I}$ and ${ }^{11} \widetilde{F}_{I}$ for coordinates in $\widetilde{\mathcal{Q}}_{I}$ is forward complete, i.e. the maximal integral curve through any initial condition is defined for all positive time.

Recall from the rigid body and unconstrained massless assumptions (A1 \& A6) that the configuration space, $\mathcal{Q}$, is a manifold without boundary. Thus the major obstacle to verifying Assumption A7 lies in preventing finite-time "escape" from the state space $T \mathcal{Q}$, e.g. because the velocity grows without bound or there are "open edges" in the configuration manifold (i.e., the manifold is not compact). If the configuration manifold were compact, then it would suffice to impose a global bound on the magnitude of the vector field in (12). If the configuration space were instead Euclidean, $\mathcal{Q}=\mathbb{R}^{n}$, then it would suffice to impose a global Lipschitz continuity condition on the vector field in (12). We note that configuration obstacles such as joint limits or self-intersections are treated as constraints in Section 3 , and hence pose no obstacle to satisfying the above boundarylessness and completeness conditions on the configuration space.

However, since in examples of interest the configuration space is neither compact nor a vector space (as noted after Assumption A1), we often require a more general condition. One such condition is obtained from (Ballard 2000, Thm. 10); since we rely on this sufficient condition elsewhere in the paper, we transcribe it explicitly into our notation as follows. When $\mathcal{Q}$ is a complete connected configuration manifold and $\overline{\mathbf{M}}$ is a nondegenerate inertia tensor (i.e., at every $\mathbf{q} \in \mathcal{Q}$ the coordinate representation of $\overline{\mathbf{M}}(\mathbf{q})$ is invertible, thus here precluding the possibility of massless limbs, Assumption A5), we let $d_{\overline{\mathbf{M}}}: \mathcal{Q} \times \mathcal{Q} \rightarrow \mathbb{R}$ denote the distance metric induced by the Riemannian metric $\langle\cdot, \cdot\rangle_{\overline{\mathbf{M}}}$ associated with $\overline{\mathbf{M}}$ (Lee 1997, Ch. 6). For any vector $\dot{\mathbf{q}} \in T_{\mathbf{q}} \mathcal{Q}$ we define $|\dot{\mathbf{q}}|_{\overline{\mathbf{M}}}:=\langle\dot{\mathbf{q}}, \dot{\mathbf{q}}\rangle_{\overline{\mathbf{M}}}$. For any covector $\mathbf{f} \in T_{\mathbf{q}}^{*} \mathcal{Q}$ we define $|\mathbf{f}|_{\overline{\mathbf{M}}^{-1}}:=\left|\mathbf{f}^{\#}\right|_{\overline{\mathbf{M}}}$, where $\mathbf{f}^{\#} \in T_{\mathbf{q}} \mathcal{Q}$ is the vector obtained by "raising an index" (in coordinates, $\mathbf{f}^{\#}=\overline{\mathbf{M}}^{-1} \mathbf{f}^{T}$ ) (Lee 1997, Ch. 3).

Lemma 6. If the ambient configuration space $\mathcal{Q}$ is a complete connected Riemannian manifold, $\overline{\mathbf{M}}$ is a nondegenerate inertia tensor, and the magnitude of $\Upsilon_{I}-\overline{\mathbf{N}}_{I}$ grows at most linearly with velocity and distance from some (hence any) point in $\mathcal{Q}$, i.e. if there exists $C \in \mathbb{R}, \mathbf{q}_{0} \in \mathcal{Q}$ such that,

$$
\forall(\mathbf{q}, \dot{\mathbf{q}}) \in T \mathcal{Q}:\left|\Upsilon_{I}(\mathbf{q}, \dot{\mathbf{q}})-\overline{\mathbf{N}}_{I}(\mathbf{q}, \dot{\mathbf{q}})\right|_{\overline{\mathbf{M}}^{-1}} \leq C\left(1+|\dot{\mathbf{q}}|_{\overline{\mathbf{M}}}+d_{\overline{\mathbf{M}}}\left(\mathbf{q}_{0}, \mathbf{q}\right)\right),
$$

then the flow associated with the vector field (12) is forward complete, i.e. the maximal integral curve through any initial condition is defined for all positive time, and hence Assumption A7 is satisfied.

Proof. This is simply an application of (Ballard 2000, Thm. 10) in the absence of unilateral constraints. 
We expect this condition to be met by any model based on a physical system, and is trivially met if there is a global bound on the magnitude of the applied, $\Upsilon_{I}$, and potential, $\overline{\mathbf{N}}_{I}$, forces (whereas, notice, the necessarily unbounded Coriolis and centripetal forces are accounted for by the Lemma and require no further consideration).

Unfortunately this condition assumes that the inertia tensor $\overline{\mathbf{M}}$ is nondegenerate, precluding the presence of massless limbs (Assumption A5). Allowing instead for a degenerate inertia tensor but enforcing the unconstrained massless limb assumption (A6), we now describe a set of sufficient conditions that ensure Assumption A7 holds.

Lemma 7. Suppose that in each contact mode I the active constraints are either holonomic or integrable (Murray et al. 1994, Sec. 6.1.1), meaning that there exists a reduced configuration manifold $\mathcal{Y}_{I}$ (i.e., generalized coordinates) such that every point in $\mathcal{Q}_{I}$ lies in the image of an embedding $h: \mathcal{Y}_{I} \rightarrow \mathcal{Q}_{I}$ that is invariant under (12) (Johnson and Koditschek 2013a, Sec. G) and restricted to which the reduced inertia tensor (Johnson and Koditschek 2013a, Eqn. 36) is nondegenerate.

If the hypotheses in Lemma 6 are satisfied for $\mathcal{Y}_{I}$, its reduced inertia tensor, and its reduced dynamics (Johnson and Koditschek 2013a, Eqn. 34), and furthermore the vector field $\widetilde{F}_{I}$ governing unconstrained massless limbs is forward complete and uncoupled from the massive or constrained coordinates, i.e.,

$$
\mathbf{T} \mathbf{q}_{I}=\operatorname{Dh}\left(\mathbf{T y}_{I}\right), \dot{\widetilde{\mathbf{q}}}_{I}=\widetilde{F}_{I}\left(\widetilde{\mathbf{q}}_{I}\right)
$$

then Assumption A7 is satisfied.

Proof. We seek to define a forward-complete flow $\phi_{I}:[0, \infty) \times T \mathcal{Q}_{I} \rightarrow T \mathcal{Q}_{I}$ consistent with the vector field in (12). Let $h: \mathcal{Y}_{I} \rightarrow \mathcal{Q}_{I}$ denote the embedding associated with the reduced coordinates (Johnson and Koditschek 2013a, Sec. G). Apply Lemma 6 to the reduced system to obtain a forward-complete flow $\widetilde{\phi}_{I}:[0, \infty) \times T \mathcal{Y}_{I} \rightarrow T \mathcal{Y}_{I}$. Then since (Ballard 2000, Prop. 3) implies $D h$ maps integral curves from the reduced state space to the original, for all $t \in[0, \infty)$ and $\mathbf{T y} \in T \mathcal{Y}_{I}$, defining $\phi(t, D h(\mathbf{T y}))=D h \circ \widetilde{\phi}(t, \mathbf{T y})$ yields the desired forward-complete flow on $\mathcal{Q}_{I}$.

Lemmas $6 \& 7$ provide sufficient conditions guaranteeing that certain systems with either full rank inertia tensors or only holonomic constraints satisfy Assumption A7 - in the most general case, however, this remains an assumption. We speculate that it is possible to derive a condition analogous to (19) using concepts from singular Riemannian geometry Hermann (1973) that ensure the existence of a forward-complete flow in the presence of nonintegrable constraints and a singular inertia tensor.

\subsection{Impulsive Dynamics}

Define the touchdown predicate, $\mathrm{TD}: \mathcal{K}_{n} \times T \mathcal{Q} \rightarrow \mathbb{B}$, where $\mathbb{B}:=\{$ True, False $\}$, as,

$$
\mathrm{TD}(k, \mathbf{T q}):=\mathbf{a}_{k}(\mathbf{q})=0 \wedge \mathbf{A}_{k}(\mathbf{q}) \dot{\mathbf{q}}<0,
$$

so that $\operatorname{TD}(k, \mathbf{T q})$ is true only at those points $\mathbf{q}$ where contact $k$ should be considered for addition (in a manner to be qualified in Theorem 2 by the impulse-velocity complementarity condition, (50), defined below). Furthermore, define the new touchdown predicate,

$$
\operatorname{NTD}(\mathbf{T q}):=\bigvee_{k \in \mathcal{K}_{n}} \operatorname{TD}(k, \mathbf{T q})
$$

such that $\operatorname{NTD}(\mathbf{T q})$ is true only at those states where some new constraint is impacting.

At impact into contact mode $J$, any incoming constraint velocity $\mathbf{A}_{J} \dot{\mathbf{q}}$ must be eliminated. Here, we assume a Newtonian impact law, e.g. (Chatterjee and Ruina 1998, Eqn. 3) or (Featherstone 2008, Eqn. 11.65), that is,

Assumption $\mathbf{A 8}$ (Plastic Impact). Impacts are plastic (inelastic), occur instantaneously, and their effect described by an algebraic equation (23), defined below.

In general, $\Delta \dot{\mathbf{q}}_{J}:=\dot{\mathbf{q}}_{J}^{+}-\dot{\mathbf{q}}^{-}$, the instantaneous change in velocity from $\dot{\mathbf{q}}^{-}$in contact mode $I$ before impact to $\dot{\mathbf{q}}_{J}^{+}$ in contact mode $J$ after impact, is modeled as, $\Delta \dot{\mathbf{q}}_{J}=-(1+e) \mathbf{A}_{J}^{\dagger T} \mathbf{A}_{J} \dot{\mathbf{q}}^{-}$(recall that $\mathbf{A}^{\dagger T}: T \mathcal{C} \rightarrow T \mathcal{Q}$ maps velocities in the contact frames to velocities of the system state). The coefficient of restitution, $e$, may be defined in any of the usual ways, however throughout this paper plastic impact $(e=0)$ is assumed. We restrict to plastic impacts as we believe it to be more relevant to most robotics applications, and since ambiguities arise when an elastic impact occurs 
in a system with multiple active constraints: different choices of impact model can yield distinct post-impact velocities (see Section 1.3.4). For plastic impacts, the post-impact velocity in mode $J$ is,

$$
\dot{\mathbf{q}}_{J}^{+}=\left(\mathbf{I d}-\mathbf{A}_{J}^{\dagger T} \mathbf{A}_{J}\right) \dot{\mathbf{q}}^{-}=\overline{\mathbf{M}}_{J}^{\dagger} \overline{\mathbf{M}} \dot{\mathbf{q}}^{-},
$$

where the final simplification follows from (11) and matches (Westervelt et al. 2003, Eqn. 9). The body impulse in configuration coordinates is,

$$
\mathbf{P}_{J}:=-\overline{\mathbf{M}}\left(\dot{\mathbf{q}}_{J}^{+}-\dot{\mathbf{q}}^{-}\right) \text {. }
$$

The contact impulse (i.e., the impulse at the contact points that induces the desired change in velocity to agree with the new contact mode $J$ ) is,

$$
\widehat{\mathbf{P}}_{J}:=\mathbf{A}_{J}^{\dagger} \mathbf{P}_{J}=\mathbf{A}_{J}^{\dagger} \overline{\mathbf{M}} \mathbf{A}_{J}^{\dagger T} \mathbf{A}_{J} \dot{\mathbf{q}}^{-}=-\Lambda_{J} \mathbf{A}_{J} \dot{\mathbf{q}}^{-}=\mathbf{A}_{J}^{\dagger} \overline{\mathbf{M}} \dot{\mathbf{q}}^{-},
$$

where recall that $\mathbf{A}_{J}, \mathbf{A}_{J}^{\dagger}, \overline{\mathbf{M}}$, and $\Lambda_{J}$ are functions of the state $\mathbf{q}$ (which does not change during impact, i.e. $\mathbf{q}^{+}=\mathbf{q}^{-}$), and the impulses, $\mathbf{P}_{J}$ and $\widehat{\mathbf{P}}_{J}$, are also functions of the incoming velocity, $\dot{\mathbf{q}}^{-}$. The final simplification arises from (11), matches (Westervelt et al. 2003, Eqn. 10), and is used in Section 2.7.

Lemma 8. When $\overline{\mathbf{M}}$ is invertible, contact impulse (25) into contact mode J is equivalent to the non-degenerate plastic impact law,

$$
\widehat{\mathbf{P}}_{J}=\left(\mathbf{A}_{J} \overline{\mathbf{M}}^{-1} \mathbf{A}_{J}^{T}\right)^{-1} \mathbf{A}_{J} \dot{\mathbf{q}}^{-},
$$

as listed e.g. in (Chatterjee and Ruina 1998, Eqn. 3).

As with the proof of Lemma 5, the result may be seen by substituting (15) or (16), the explicit solution to (8) when $\overline{\mathbf{M}}$ is invertible, into (25), as worked out in Appendix B.3.

\subsection{Complementarity}

We now introduce the classical complementarity problems for forces and impulses at the contact points, and provide a reformulation that allows massless limbs. We begin with a general statement of the complementarity property (as in e.g. Cottle 1968, Ingleton 1966, Lötstedt 1982, van der Schaft and Schumacher 1998), then subsequently specialize in Sections 2.6.1 and 2.6.2 to formulations of force-acceleration and impulse-velocity complementarity. Both versions have the general form of seeking real vectors $\mathbf{y}$ and $\mathbf{z}$ such that,

$$
\mathbf{y} \geq 0, \quad \mathbf{z} \geq 0, \quad \mathbf{y}^{T} \mathbf{z}=0,
$$

(where for a vector $\mathbf{y}, \mathbf{y} \geq 0 \Leftrightarrow \mathbf{y}_{i} \geq 0 \forall i$ ) subject to some problem-specific constraints. While the most general problem is uncoupled, that is $\mathbf{y}$ and $\mathbf{z}$ may be chosen arbitrarily so long as they satisfy (27), the cases we consider here are coupled by these problem-specific constraints (Pang et al. 1996, Sec. 3). In the linear complementarity problem (LCP), for instance, the coupling constraint has the form $\mathbf{z}:=\mathbf{A y}+\mathbf{c}$ (e.g. Brogliato et al. 2002, Eqn. 8). The functional relationships between $\mathbf{y}$ and $\mathbf{z}$ for the complementarity problems in this paper is in general nonlinear (as discussed in the rest of this section). Since the relation of interest is generally problem-specific and index dependent in an essential way, we introduce temporarily an abstract scalar relation, $\unrhd$ instead of $\succeq$ or $\geq$ and similarly $\triangleright$ instead of $\succ$ or $>$, whose different instantiations are prescribed in the force-acceleration and impulse-velocity versions of the problem, respectively. For simplicity of notation we use $=$ as the corresponding equality relation.

Solutions to this problem produce a natural bipartition $\left(J, J^{C}\right)$ on some index set, $\mathcal{I}$, the scope (some subset of the universal scope $\mathcal{K}$, to be discussed below), where $J=\left\{j \in \mathcal{I}: z_{j}=0\right\}$ and $J^{C}=\mathcal{I} \backslash J=\left\{j \in \mathcal{I}: z_{j} \triangleright 0\right\}$. Here, the role of $\mathbf{y}$ and $\mathbf{z}$ are played by physically determined functions of a specified ("incoming") state, $\mathbf{T q}^{-}=\left(\mathbf{q}^{-}, \dot{\mathbf{q}}^{-}\right)$, to yield an "outgoing" bipartition $\left(J, J^{C}\right)$ of the indexing scope, $\mathcal{I}$. The indexing scope is a function only of the incoming continuous state, $\mathcal{I}: T \mathcal{Q} \rightarrow 2^{\mathcal{K}}$, as defined in (33).

It should now be clear that for this paper the complementarity problem is reduced to finding the unknown bipartition $\left(J, J^{C}\right)$, also known as the mode selection problem (van der Schaft and Schumacher 1998), as opposed to finding the values of the two complementarity vectors directly, e.g. (Cottle 1968). Namely, given an index set $\mathcal{I}$, two functions $Y, Z: 2^{\mathcal{I}} \times T \mathcal{Q} \rightarrow \mathbb{R}^{|\mathcal{I}|}$ that map a subset $J \in 2^{\mathcal{I}}$ into a Euclidian space with dimension equal to the size of the index 
set, and a generic relation $\triangleright$ (to be instantiated as $\succ$ or $>$ in the following sections ${ }^{12}$ ), we require a solution to a set of constraint equations of the form,

$$
\begin{array}{lll}
Y_{j}\left(J, \mathbf{T q}^{-}\right) \unrhd 0, & Z_{j}\left(J, \mathbf{T q}^{-}\right)=0, & \forall j \in \mathcal{I} \cap J, \\
Y_{k}\left(J, \mathbf{T q}^{-}\right)=0, & Z_{k}\left(J, \mathbf{T q}^{-}\right) \triangleright 0, & \forall k \in \mathcal{I} \backslash J,
\end{array}
$$

(where by definition, $\mathcal{I} \cap J=J$ ). For the complementarity problems of interest in this paper, the equality constraints in (28)-(29) hold for all arguments $\left(J, \mathbf{T q}^{-}\right) \in 2^{\mathcal{I}} \times T \mathcal{Q}$ by construction (enforced, e.g., by the flow (12) in the forceacceleration version, and by the impact map (25) in the impulse-velocity version).

The complementarity problem as stated thus far is not explicitly coupled (Pang et al. 1996, Sec. 3), i.e. it places no requirements on the relationship between $Y_{k}$ and $Z_{k}$ other than their common dependence on $J$ and $\mathbf{T q}^{-}$, which is why existence and uniqueness properties are challenging to define in general. Furthermore, this necessitates the evaluation of both $Y_{k}$ and $Z_{k}$ for constraints $k$ that are not in $J$. With the possibly massless limbs in our setting, the evaluation of $Z_{k}$ is not always possible as the concept of a separation velocity or acceleration is poorly defined (once such a contact point has lifted off the ground the corresponding joints must be dropped from the state according to the unconstrained massless limb assumption, A6). Thus the specifics of $Z_{k}$ in the problems considered in this paper necessitate an alternate formulation that takes advantage of the coupling between $Y_{k}$ and $Z_{k}$, as the inequality constraints have the property that,

$$
Z_{k}\left(J, \mathbf{T q}^{-}\right) \triangleright 0 \Leftrightarrow Y_{k}\left(J \cup\{k\}, \mathbf{T} \mathbf{q}^{-}\right) \not 0,
$$

(the importance of the $(J \cup\{k\})$ mode was first noted in (Ingleton 1966, Eqn. 1.7.3)). This suggests the combined expression,

$$
(k \in J) \Leftrightarrow\left(Y_{k}\left(J \cup\{k\}, \mathbf{T q}^{-}\right) \unrhd 0\right), \quad \forall k \in \mathcal{I},
$$

which is equivalent to (28) \& (29),

Lemma 9. The separate relational statements of the complementarity problem, (28)-(29), are equivalent to a single biconditional statement, (31), provided that the complementary functions $Y$ and $Z$ satisfy (30).

Proof. First note that for $k \in J$ it is trivially true that $J \cup\{k\}=J$ and so (31) simplifies to the first condition of (28). For $k \notin J$, the expression in (31) along with the substitution of (30) reduces to the second condition of (29).

Expressing (31) as a predicate PRED : $2^{\mathcal{K}} \times T \mathcal{Q} \rightarrow \mathbb{B}$,

$$
\operatorname{PRED}\left(J, \mathbf{T q}^{-}\right)=(J \subseteq \mathcal{I}) \bigwedge_{k \in \mathcal{I}}(k \in J) \Leftrightarrow\left(Y_{k}\left(J \cup\{k\}, \mathbf{T q}^{-}\right) \unrhd 0\right) .
$$

We denote by,

$$
\mathrm{CP}_{\text {PRED }}: T \mathcal{Q} \rightarrow 2^{\mathcal{K}}: \mathbf{T q}^{-} \mapsto J
$$

the implicit function that solves this set of constraints for the unknown required bipartition, where PRED varies with the particular instances as determined by the appropriate form of $Y_{k}$. Note that while the codomain is $2^{\mathcal{K}}$, the solution is always a member of $2^{\mathcal{I}}$.

The existence of this implicit function (32) (i.e., the existence and uniqueness of a solution, $J$, to the mode selection problem) is in the most general cases an additional assumption ${ }^{13}$ (see Assumption A9 and A10, below), although the specific complementarity problems in this section (i.e., based on the relationship of the specific functions $Y$ and $Z$ used in these cases) in the absence of friction reduce down to the conventional LCP problem and so existence and uniqueness has been proven in e.g. Cottle (1968), Ingleton (1966), Lötstedt (1982), van der Schaft and Schumacher (1998).

The motivating literature and related work discussed in Section 1.3 generally imposes two complementarity conditions on rigid body dynamics models. The force-acceleration (FA) variant of (28)-(29), presented in (35)-(36), stipulates that there cannot be both a continuous time contact force and a separation acceleration at the same contact

\footnotetext{
${ }^{12}$ Recall that the relation may, in the case of $\succ$, depend on the vector field at that point.

${ }^{13}$ However note that the remainder of this paper only requires a unique choice of a solution that satisfies the predicate if multiple solutions exist.
} 
point, and is widely considered to arise from fundamental physical reasoning. In the present setting, FA complementarity governs exclusively the nature of liftoff events (and extended in Section 2.8 to stick/slip events) wherein the number of active contacts (i.e., cardinality of the mode set) is reduced for reasons discussed in Section 2.6.1. In contrast, during instantaneous impact events the contact forces have no time to perform work. Instead, the impulsevelocity (IV) variant of (28)-(29), presented in (46)-(47), precludes a simultaneous impact-induced contact impulse and separation velocity at the same contact point. This constraint is known not to follow inevitably from the rational mechanics of rigid body models (Chatterjee 1999), but represents a commonly exploited algorithmic simplification that we embrace in this inelastic model at the possible expense of consistency with elastic impact models in the limit. In the present setting, IV complementarity governs exclusively the nature of touchdown events wherein one or more new contacts become active (i.e., cardinality of the mode set is increased) for reasons discussed in Section 2.6.2.

\subsubsection{Force-Acceleration (FA) Complementarity}

For continuous time contact forces, when $\operatorname{NTD}(\mathbf{T q})$ is false and therefore $\mathbf{P}=0$ when one or more contact constraints violate the unilateral constraint cone ${ }^{14} \mathbf{U}$, some constraints lift off and must be removed from the set of active constraints, resulting in a transition to a new mode. Determining that next mode sets up a complementarity problem over the existing contact mode $I$ between the unilateral constraint cone, $\mathbf{U}_{k}(\boldsymbol{\lambda})$, if the contact is kept, and the separation acceleration $\frac{d}{d t} \mathbf{A}_{k} \dot{\mathbf{q}}=\mathbf{A}_{k} \ddot{\mathbf{q}}+\dot{\mathbf{A}}_{k} \dot{\mathbf{q}}$ if it is removed (recall that as an active constraint the state velocity is initially $\mathbf{A}_{k} \dot{\mathbf{q}}=0$ ). The full scope of contact constraints that should be considered is the set of all contacts which are "touching", i.e. those whose normal direction component have zero contact distance and a non-separating velocity ${ }^{15}$,

$$
\begin{aligned}
\mathcal{I}(\mathbf{T q}): & =\left\{i \in \mathcal{K}: \mathbf{a}_{\alpha(i)}(\mathbf{q})=0 \wedge \mathbf{A}_{\alpha(i)}(\mathbf{q}) \dot{\mathbf{q}} \leq 0\right\} \\
& =\left\{i \in \mathcal{K}:\left(\mathbf{a}_{\alpha(i)}(\mathbf{q})=0 \wedge \mathbf{A}_{\alpha(i)}(\mathbf{q}) \dot{\mathbf{q}}=0\right) \vee \operatorname{TD}(\alpha(i), \mathbf{T} \mathbf{q})\right\} .
\end{aligned}
$$

Recall that force-acceleration complementarity only holds when $\operatorname{NTD}(\mathbf{T q})$ is false and so the final condition applies here. Furthermore, while the full scope is formally required and does not depend on the active mode, numerically it suffices to check $I \subseteq \mathcal{I}$ - this reduced scope eliminates the numerical challenge of checking the exact equality of (33). See Section 4.4 for a further discussion of this simplification and other numerical implementation details.

For transition into $J$, consider contact force (13) both in $J$ but also in the alternative mode $J \cup\{k\}$ where contact $k$ is maintained (the reason for this alternative mode is clear in Theorem 1),

$$
\begin{array}{rlrl}
\mathbf{U}_{j}\left(\lambda_{J}\right) & \succeq 0, \mathbf{A}_{j} \ddot{\mathbf{q}}+\dot{\mathbf{A}}_{j} \dot{\mathbf{q}} \equiv 0, & & \forall j \in \mathcal{I} \cap J, \\
\mathbf{U}_{k}\left(\lambda_{J}\right) \equiv 0, \mathbf{U}_{k}\left(\lambda_{J \cup\{k\}}\right) \prec 0, & & \forall k \in \mathcal{I} \backslash J,
\end{array}
$$

where the identically zero constraints are guaranteed to hold in consequence of the dynamics governing mode $J$, namely, the invariance of the flow $\left(\mathbf{A}_{j} \ddot{\mathbf{q}}+\dot{\mathbf{A}}_{j} \dot{\mathbf{q}} \equiv 0 \forall j \in J\right.$ by (12)) and the Lagrange multipliers $\left(\mathbf{U}_{k}\left(\lambda_{J}\right) \equiv 0 \forall k \notin J\right.$ by (13)). Note the importance here of the trending positive/negative conditions (Definition 1) - in general it is not sufficient to simply check the sign of the contact force but possibly higher derivatives as well. For example, in Figure 2, cases (c) and (d), assume the particle is sliding along the constraint from left to right. At the moment the particle reaches the origin, the contact force is zero. However in (c) the contact force is trending negative and the constraint should be removed, while in (d) the contact force is trending positive and it should be maintained.

Constraints (35), (36) can be simplified into a form analogous to (30), hence, by Lemma 9,

$$
(k \in J) \Leftrightarrow\left(\mathbf{U}_{k}\left(\lambda_{J \cup\{k\}}\right) \succeq 0\right), \quad \forall k \in \mathcal{I},
$$

or as the predicate $\mathrm{FA}: 2^{\mathcal{K}} \times T \mathcal{Q} \rightarrow \mathbb{B}$,

$$
\operatorname{FA}\left(J, \mathbf{T q}^{-}\right)=(J \subseteq \mathcal{I}) \bigwedge_{k \in \mathcal{I}}(k \in J) \Leftrightarrow\left(\mathbf{U}_{k}\left(\lambda_{J \cup\{k\}}\right) \succeq 0\right),
$$

for which we write the associated implicit function solution, following (32), as, $\mathrm{CP}_{\mathrm{FA}}\left(\mathbf{T q}^{-}\right)=J$.

This formulation of the force-acceleration complementarity problem is required to allow for massless limbs, for which a separation acceleration is not well defined. However when the separating acceleration is defined,

\footnotetext{
${ }^{14}$ Recall from Section 2.2 that $\mathbf{U}$ in the normal direction is -1 according to the frame conventions of (Johnson and Koditschek 2013a, Eqn. 76, 78).

${ }^{15}$ Note that thus far only normal direction constraints have been considered, however Section 2.8 extends this to include tangential (sliding friction) constraints and this scope is defined in this general way in order to apply there as well.
} 
Theorem 1. The non-penetrating acceleration condition at a contact $k$ after liftoff into mode $J, \mathbf{A}_{k} \ddot{\mathbf{q}}+\dot{\mathbf{A}}_{k} \dot{\mathbf{q}} \succ 0$ (when such an acceleration is well defined), is equivalent to a trending negative contact force $\mathbf{U}_{k}\left(\lambda_{K}\right) \prec 0$ in mode $K:=J \cup\{k\}$. In other words, (35)-(36) are equivalent to the usual formulation (e.g. Brogliato et al. 2002, Eqn. 10),

$$
\begin{array}{rlrl}
\mathbf{U}_{j}\left(\lambda_{J}\right) & \succeq 0, \mathbf{A}_{j} \ddot{\mathbf{q}}+\dot{\mathbf{A}}_{j} \dot{\mathbf{q}} \equiv 0, & & \forall j \in \mathcal{I} \cap J, \\
\mathbf{U}_{k}\left(\lambda_{J}\right) \equiv 0, \mathbf{A}_{k} \ddot{\mathbf{q}}+\dot{\mathbf{A}}_{k} \dot{\mathbf{q}} \succ 0, & & \forall k \in \mathcal{I} \backslash J .
\end{array}
$$

Proof. Recall from Section 2.1 that $\pi_{I}$ is the canonical projection onto the linear subspace spanned by the coordinate axes indexed by $I$, and assume without loss of generality that $k$ is the final index in $K$ such that,

$$
\mathbf{A}_{K}:=\pi_{K} \mathbf{A}_{\mathcal{K}}=\left[\begin{array}{l}
\mathbf{A}_{J} \\
\mathbf{A}_{k}
\end{array}\right]=\left[\begin{array}{c}
\pi_{J} \mathbf{A}_{\mathcal{K}} \\
\pi_{k} \mathbf{A}_{\mathcal{K}}
\end{array}\right]
$$

The constraint cone, following (Johnson and Koditschek 2013a, Eqn. 78), applied to the contact forces, (13), is (see Appendix B for details, in particular (117) expanding $\mathbf{A}_{K}^{\dagger}$ and $\Lambda_{K}$ ),

$$
\begin{aligned}
\mathbf{U}_{k}\left(\lambda_{K}\right) & =-\pi_{k}\left(\mathbf{A}_{K}^{\dagger}(\Upsilon-\overline{\mathbf{C}} \dot{\mathbf{q}}-\overline{\mathbf{N}})-\Lambda_{K} \dot{\mathbf{A}}_{K} \dot{\mathbf{q}}\right) \\
& =-\frac{\mathbf{A}_{k} \overline{\mathbf{M}}_{J}^{\dagger}}{\mathbf{A}_{k} \overline{\mathbf{M}}_{J}^{\dagger} \mathbf{A}_{k}}(\Upsilon-\overline{\mathbf{C}} \dot{\mathbf{q}}-\overline{\mathbf{N}})-\frac{1}{\mathbf{A}_{k} \overline{\mathbf{M}}_{J}^{\dagger} \mathbf{A}_{k}^{T}}\left[\mathbf{A}_{k} \mathbf{A}_{J}^{\dagger T}-1\right]\left[\begin{array}{c}
\dot{\mathbf{A}}_{J} \\
\dot{\mathbf{A}}_{k}
\end{array}\right] \dot{\mathbf{q}} \\
& =-\frac{\mathbf{A}_{k} \overline{\mathbf{M}}_{J}^{\dagger}(\Upsilon-\overline{\mathbf{C}} \dot{\mathbf{q}}-\overline{\mathbf{N}})-\mathbf{A}_{k} \mathbf{A}_{J}^{\dagger T} \dot{\mathbf{A}}_{J} \dot{\mathbf{q}}+\dot{\mathbf{A}}_{k} \dot{\mathbf{q}}}{\mathbf{A}_{k} \overline{\mathbf{M}}_{J}^{\dagger} \mathbf{A}_{k}^{T}}
\end{aligned}
$$

while the separating acceleration for constraint $k$ in mode $J$ is, using (12),

$$
\mathbf{A}_{k} \ddot{\mathbf{q}}_{J}+\dot{\mathbf{A}}_{k} \dot{\mathbf{q}}_{J}=\mathbf{A}_{k}\left(\overline{\mathbf{M}}_{J}^{\dagger}(\Upsilon-\overline{\mathbf{C}} \dot{\mathbf{q}}-\overline{\mathbf{N}})-\mathbf{A}_{J}^{\dagger T} \dot{\mathbf{A}}_{J} \dot{\mathbf{q}}\right)+\dot{\mathbf{A}}_{k} \dot{\mathbf{q}}=-\left(\mathbf{A}_{k} \overline{\mathbf{M}}_{J}^{\dagger} \mathbf{A}_{k}^{T}\right) \mathbf{U}_{k}\left(\lambda_{K}\right)
$$

Since the denominator in (44) is a positive scalar function of state (as shown in Appendix B, (118)), by Definition 1 and Lemma 2 a trending positive separation acceleration, $\mathbf{A}_{k} \ddot{\mathbf{q}}+\dot{\mathbf{A}}_{k} \dot{\mathbf{q}}$ implies a trending negative contact force, $\mathbf{U}_{k}\left(\lambda_{K}\right)$, and vice-versa, and therefore (35)-(36) are equivalent conditions to (39)-(40).

Furthermore, with or without a full rank inertia tensor, we assume the existence of a unique solution to the forceacceleration complementarity problem,

Assumption A9 (Force-Acceleration Complementarity). The force-acceleration complementarity constraints, FA, (38), always admit an implicit function, $\mathrm{CP}_{\mathrm{FA}}$, over the entire state space $T \mathcal{Q}$, even under the frictional properties that follow Assumption A12. That solution correctly captures the behavior of the physical system.

While there has been a long line of literature (e.g., (van der Schaft and Schumacher 1998, Ex. 3.3) ${ }^{16}$ ) that proves that this is always true for independent, plastic, frictionless contacts, no result has been found that covers the limited frictional conditions introduced in 2.8. We impose this condition in support of the Theorems in Section 3.

\subsubsection{Impulse-Velocity (IV) Complementarity}

Impact at one contact location can cause another contact to break, as the contact impulse must obey the unilateral constraint cone, $\mathbf{U}_{j}\left(\widehat{\mathbf{P}}_{J}\right) \geq 0 \forall j \in J$, i.e. both that the impulse in the normal direction be positive (non-adhesive) and that the tangential impulse lie in the friction cone (Chatterjee and Ruina 1998). Any contact point that would have violated that requirement must be dropped from the active contact mode.

In addition the post-impact velocity must not allow the removed contact point to leave with a penetrating velocity (i.e., the impulse cannot result in a velocity "into" the surface). However in the case of massless legs a positive separation velocity is always achievable. As an alternative requirement that is based only on impulses ${ }^{17}$, consider

\footnotetext{
${ }^{16}$ In the language of that paper, this is a Dynamic Complementarity Problem (DCP), and note that (van der Schaft and Schumacher 1998, Eqn. 33) asserts complementarity with the base constraint (which they call $y=C(q)$ ), but here the position and velocity are already zero, i.e. $\forall j \in I, \mathbf{a}_{j}(\mathbf{q})=\mathbf{A}_{j}(\mathbf{q}) \dot{\mathbf{q}}=0$, and so the acceleration is the first degree that must be checked.

${ }^{17}$ Note that this formulation based only on impulses also simplifies the inclusion of the pseudo-impulse condition (58).
} 
the contact impulse (25), $\widehat{\mathbf{P}}_{J}$ (associated with the passage from contact $I$ to contact $J$ ), but also the contact impulse $\widehat{\mathbf{P}}_{J \cup\{k\}}$ (associated with the passage from contact $I$ to alternative mode $J \cup\{k\}$ where contact $k$ is maintained). These impulses, along with the post-impact velocity (23), $\mathbf{A}_{J} \dot{\mathbf{q}}^{+}$, must satisfy,

$$
\begin{array}{ll}
\mathbf{U}_{j}\left(\widehat{\mathbf{P}}_{J}\right) \geq 0, \mathbf{A}_{j} \dot{\mathbf{q}}^{+}=0, & \forall j \in \mathcal{I} \cap J, \\
\mathbf{U}_{k}\left(\widehat{\mathbf{P}}_{J}\right)=0, \mathbf{U}_{k}\left(\widehat{\mathbf{P}}_{J \cup\{k\}}\right)<0, & \forall k \in \mathcal{I} \backslash J,
\end{array}
$$

where the scope, $\mathcal{I}$, is again formally the set of all touching constraints, (33). However in numerical simulation it is sufficient to check only the active constraints as well as those touching down ${ }^{18},(21)$,

$$
\mathcal{I}_{\mathrm{IV}}:=I \cup\{i \in \mathcal{K} \backslash I: \operatorname{TD}(\alpha(i), \mathbf{T q})\} \subseteq \mathcal{I} .
$$

Note that the equality constraints are enforced by the impact law (25), and so, by Lemma 9, constraints (46)-(47) reduce to,

$$
(k \in J) \Leftrightarrow\left(\mathbf{U}_{k}\left(\widehat{\mathbf{P}}_{J \cup\{k\}}\right) \geq 0\right), \quad \forall k \in \mathcal{I} .
$$

or as the predicate IV $: 2^{\mathcal{K}} \times T \mathcal{Q} \rightarrow \mathbb{B}$

$$
\operatorname{IV}\left(J, \mathbf{T q}^{-}\right)=(J \subseteq \mathcal{I}) \bigwedge_{k \in \mathcal{I}}(k \in J) \Leftrightarrow\left(\mathbf{U}_{k}\left(\widehat{\mathbf{P}}_{J \cup\{k\}}\right) \geq 0\right),
$$

whose solution, following (32), is denoted, $\mathrm{CP}_{\mathrm{IV}}\left(\mathbf{T q}^{-}\right)=J$.

As with the force-acceleration complementarity problem, this formulation of the impulse-velocity complementarity problem is required to allow for massless limbs, for which a separating velocity is not well defined. However when the separating velocity is defined,

Theorem 2. The non-penetrating velocity condition at a contact $k$ after impact into mode $J, \mathbf{A}_{k} \dot{\mathbf{q}}^{+}>0$ (where such a velocity is well defined), is equivalent to a negative contact impulse, $\mathbf{U}_{k}\left(\widehat{\mathbf{P}}_{K}\right)<0$, at impact into mode $K:=J \cup\{k\}$. In other words, (46)-(47) are equivalent to the usual formulation (e.g. Brogliato et al. 2002, Eqn. 9),

$$
\begin{aligned}
\mathbf{U}_{j}\left(\widehat{\mathbf{P}}_{J}\right) \geq 0, \mathbf{A}_{j} \dot{\mathbf{q}}^{+}=0, & \forall j \in \mathcal{I} \cap J, \\
\mathbf{U}_{k}\left(\widehat{\mathbf{P}}_{J}\right)=0, \mathbf{A}_{k} \dot{\mathbf{q}}^{+}>0, & \forall k \in \mathcal{I} \backslash J .
\end{aligned}
$$

Proof. Recall from Section 2.1 that $\pi_{I}$ is the canonical projection onto the linear subspace spanned by the coordinate axes indexed by $I$, and assume without loss of generality that $k$ is the final index in $K$ such that $\mathbf{A}_{K}$ is defined as in (41). The constraint cone, following (Johnson and Koditschek 2013a, Eqn. 78), applied to the contact impulse, (25), is (see Appendix B for details, in particular (117) expanding $\Lambda_{K}$ ),

$$
\mathbf{U}_{k}\left(\widehat{\mathbf{P}}_{K}\right)=\pi_{k} \Lambda_{K} \mathbf{A}_{K} \dot{\mathbf{q}}^{-}=\frac{1}{\mathbf{A}_{k} \overline{\mathbf{M}}_{J}^{\dagger} \mathbf{A}_{k}^{T}}\left[\begin{array}{ll}
\mathbf{A}_{k} \mathbf{A}_{J}^{\dagger T} & 1
\end{array}\right]\left[\begin{array}{c}
\mathbf{A}_{J} \\
\mathbf{A}_{k}
\end{array}\right] \dot{\mathbf{q}}^{-}=\frac{\mathbf{A}_{k} \mathbf{A}_{J}^{\dagger T} \mathbf{A}_{J} \dot{\mathbf{q}}^{-}-\mathbf{A}_{k} \dot{\mathbf{q}}^{-}}{\mathbf{A}_{k} \overline{\mathbf{M}}_{J}^{\dagger} \mathbf{A}_{k}^{T}}
$$

while the separating velocity for constraint $k$ after impact into mode $J$ is, using (23),

$$
\mathbf{A}_{k} \dot{\mathbf{q}}_{J}^{+}=\mathbf{A}_{k} \dot{\mathbf{q}}^{-}-\mathbf{A}_{k} \mathbf{A}_{J}^{\dagger T} \mathbf{A}_{J} \dot{\mathbf{q}}^{-}=-\left(\mathbf{A}_{k} \overline{\mathbf{M}}_{J}^{\dagger} \mathbf{A}_{k}^{T}\right) \mathbf{U}_{k}\left(\widehat{\mathbf{P}}_{K}\right) .
$$

Since the denominator in (53) is a positive scalar function of state (as shown in Appendix B, (118)), a positive separation velocity, $\mathbf{A}_{k} \dot{\mathbf{q}}^{+}$implies a negative contact impulse, $\mathbf{U}_{k}\left(\widehat{\mathbf{P}}_{K}\right)$, and vice-versa, and therefore (46)-(47) are equivalent conditions to (51)-(52).

Furthermore, with or without a full rank inertia tensor, we assume the existence of a unique solution to the impulsevelocity complementarity problem,

\footnotetext{
${ }^{18}$ Note that this excludes those constraints which are touching but separating, but whose post-impact velocity is penetrating. For such cases the application of this first impact puts the state into the guard for those constraints, and thus even in this pathological case the execution continues from the correct mode.
} 
Assumption A10 (Impulse-Velocity Complementarity). The impulse-velocity complementarity constraint, IV, (50) always admit an implicit function, $\mathrm{CP}_{\mathrm{IV}}$, over the entire state space $T \mathcal{Q}$, as does the modified problem including the pseudo-impulse, PIV, (58), introduced in Section 2.7, and under the frictional properties that follow Assumption A12. That solution correctly captures the behavior of the physical system.

As with the FA complementarity problem, there has been a long line of literature (e.g. Lötstedt 1982, Eqn. 2.10b) that proves that this is always true for independent, plastic, frictionless contacts, however no result has been found that covers the pseudo-impulse introduced in Section 2.7 or the limited frictional conditions introduced in Section 2.8. We impose this condition in support of the Theorems in Section 3.

\subsection{Pseudo-Impulse}

Impulses arising from impacts (both plastic and elastic) generally break existing contacts. For example an impulse imparted to the underside of a rigid block that is being pushed down onto a level surface must cause it to leave the surface for a small time interval no matter how weak the impulse or how strong the applied force. In truth the block is not rigid and the impulse is temporally distributed; modeling the resulting subtle deflections would greatly complicate the system. It appears expedient to impose a minimum threshold on impulse magnitude below which the system may be considered quasi-static and the block remains on the ground, but above which the system is dynamic and the block detaches from the substrate. This threshold could be specified directly in terms of a pre-defined limit on the system velocity or impulse magnitudes, however such limits would not take into account the magnitude of the applied force (i.e., how strongly gravity or commanded torques are holding down the rigid block). Here we want the cutoff to scale with respect to the other problem variables (such as incoming velocity, applied forces, and the contact configuration), and we show in Section 2.7.1 that this induces an implicit (variable) bound on velocity.

In this section we define an additional impulse during impact which qualitatively improves results and eliminates some Zeno phenomena. The time scaling parameter of this impulse may be thought of as a tuning parameter and while we give some physical motivation for its magnitude, the inclusion of this term is motivated primarily by improving the qualitative behavior of the numerical simulation (e.g. by excluding chattering and Zeno phenomena) in a manner that retains physical fidelity across a broad range of application settings and preserves mathematical consistency. See Section 4.3 for examples of physical situations whose physical fidelity and mathematical properties both appear to be enhanced by the introduction of such a pseudo-impulse.

Therefore we make the following new assumption about the physics of the system,

Assumption A11 (Pseudo-Impulse). The continuous time forces apply some small amount of work during the impact process.

In the usual Newtonian impact model, these forces have no effect (as shown, e.g., in ten Dam and Willems 1997, Prop. 4.3), however here we add an additional pesudo-impulse to support this assumption.

This assumption is not used in this paper to directly change the energy at any state, but rather is used within the discrete switching logic to improve the quality of make-break contact decisions (which implicitly changes the resulting kinetic energy of the system). Specifically, consider the pseudo-impulse, $\widetilde{\mathbf{P}} \in T^{*} \mathcal{C}$, that the contacts would impart on the system to resist the continuous time forces for some small time duration, $\delta_{t} \in \mathbb{R}^{+}$, during impact into mode $J$,

$$
\begin{aligned}
\overline{\mathbf{M}} \delta_{\dot{\mathbf{q}}} & :=\lim _{\delta_{t} \rightarrow 0} \int_{\delta_{t}} \overline{\mathbf{M}} \ddot{\mathbf{q}} d t \approx\left(\Upsilon-\overline{\mathbf{C}} \dot{\mathbf{q}}^{-}-\overline{\mathbf{N}}\right) \delta_{t}, \\
\widetilde{\mathbf{P}} & :=\mathbf{A}_{J}^{\dagger} \overline{\mathbf{M}} \delta_{\dot{\mathbf{q}}}=\mathbf{A}_{J}^{\dagger}\left(\Upsilon-\overline{\mathbf{C}} \dot{\mathbf{q}}^{-}-\overline{\mathbf{N}}\right) \delta_{t} .
\end{aligned}
$$

This small time $\delta_{t}$ can be regarded as the finite duration of the (actually non-instantaneous) impact process (Quinn 2005). An alternative interpretation is that $\delta_{t}$ specifies the time duration after an impulsive separation of a contact during which if that contact were to return to the active set, the qualitative behavior of the system would be improved by never considering it as having left. This interpretation, correct to first order for single contact systems, is useful when considering what value of $\delta_{t}$ should be used. In the simulations shown in this paper a magnitude of $\delta_{t}=0.03 \mathrm{~s}$ has been found to give the best results, although this value is surely dependent on the material properties, the system velocities, and the desired qualitative and quantitative behavior of the model.

This pesudo-impulse is not directly applied to the system (as in Quinn 2005), because in this model impacts occur instantaneously and the velocity displacement $\delta_{\dot{\mathbf{q}}}$ would not be uniquely determined by (56) when $\overline{\mathbf{M}}$ is singular. 
Instead the pseudo-impulse is added to the plastic impact impulse, (25), and in that way can be considered as a modified incoming momentum,

$$
\widehat{\mathbf{P}}_{K}+\widetilde{\mathbf{P}}_{K}=-\Lambda_{K} \mathbf{A}_{K} \dot{\mathbf{q}}+\mathbf{A}_{K}^{\dagger} \overline{\mathbf{M}} \delta_{\dot{\mathbf{q}}}=\mathbf{A}_{K}^{\dagger}\left(\overline{\mathbf{M}} \dot{\mathbf{q}}+\overline{\mathbf{M}} \delta_{\dot{\mathbf{q}}}\right)
$$

This modified momentum is used as an extra guard condition during impact, $\mathbf{U}(\widehat{\mathbf{P}}+\widetilde{\mathbf{P}}) \geq 0$, in addition to the usual condition, $\mathbf{U}(\widehat{\mathbf{P}}) \geq 0$, since the pseudo-impulse should not break contacts that would otherwise persist. That is, when $\delta_{t}>0$ the IV complementarity problem (50) is replaced by the predicate,

$$
\operatorname{PIV}(J, \mathbf{T q}):=\left(\operatorname{CP}_{\mathrm{IV}}(\mathbf{T q}) \subseteq J \subseteq \mathcal{I}\right) \bigwedge_{k \in \mathcal{I}}(k \in J) \Leftrightarrow\left(\mathbf{U}_{k}\left(\widehat{\mathbf{P}}_{K}\right) \geq 0 \vee \mathbf{U}_{k}\left(\widehat{\mathbf{P}}_{K}+\widetilde{\mathbf{P}}_{K}\right) \geq 0\right)
$$

whose solution, following (32), is denoted, $\mathrm{CP}_{\mathrm{PIV}}\left(\mathbf{T q}^{-}\right)=J$. Note that by construction,

$$
\forall \mathbf{T q} \in T \mathcal{Q}: \mathrm{CP}_{\mathrm{IV}}(\mathbf{T q}) \subseteq \mathrm{CP}_{\mathrm{PIV}}(\mathbf{T q})
$$

The formulation of the complementarity condition based only on impulses in (46)-(47) is key to admitting this modification, as using instead (51)-(52) would require considering both the impulsive and velocity implications of this pseudo-impulse.

The existence and uniqueness of a solution to (58) is, for the purposes of this paper, simply assumed under Assumption A10. In numerical studies, we did not encounter simulations that violated this property. However, we have not derived sufficient conditions ensuring the property holds for this modified complementarity predicate.

\subsubsection{Velocity Implications of the Pseudo-Impulse}

The IV predicate is provided as a purely logical proposition. However, its truth value varies in physically-interpretable ways with respect to variations in the base point at which it is evaluated. In the following, Lemma 10 shows that $\mathrm{CP}_{\mathrm{IV}}$ returns the same answer regardless of the impact speed along any particular impacting velocity direction, and Theorem 3 shows that $\mathrm{CP}_{\mathrm{PIV}}$ does not. See Section 4.3 for a discussion of physical implications of these results in simple example settings.

Lemma 10. Let $I \subseteq \mathcal{K},(\mathbf{q}, \dot{\mathbf{q}}) \in T \mathcal{Q}$ be such that there exists a unique $k \in \mathcal{K} \backslash$ I such that $\mathbf{a}_{k}(\mathbf{q})=0$ and $\mathbf{A}_{k}(\mathbf{q}) \dot{\mathbf{q}}<0$, i.e. the system instantaneously undergoes impact with exactly one constraint $k \in \mathcal{K}$. Define $K:=I \cup\{k\}$ and,

$$
\forall s \geq 0: \dot{\mathbf{q}}_{s}:=\left(\mathbf{I d}-(1-s) \mathbf{A}_{K}^{\dagger T} \mathbf{A}_{K}\right) \dot{\mathbf{q}}
$$

If $\delta_{t}=0$, then $J:=\mathrm{CP}_{\mathrm{IV}}(\mathbf{q}, \dot{\mathbf{q}}) \subseteq \mathcal{K}$ satisfies,

$$
\forall s>0: J=\mathrm{CP}_{\mathrm{IV}}\left(\mathbf{q}, \dot{\mathbf{q}}_{s}\right)
$$

that is the solution to the complementarity problem is the same at any impacting speed.

Proof. The impulse-velocity complementarity predicate (50) is a conjunction of propositions involving conic inequalities; since furthermore the contact impulse (25) is simply scaled (this first identity can be seen using the expansions given in Appendix B, (117), and see also (10)),

$$
\begin{aligned}
& \mathbf{A}_{J} \mathbf{A}_{K}^{\dagger T} \mathbf{A}_{K}=\mathbf{A}_{J}\left[\mathbf{A}_{J}^{\dagger T}+\overline{\mathbf{M}}_{J}^{\dagger} \ldots \quad-\overline{\mathbf{M}}_{J}^{\dagger} \ldots\right] \mathbf{A}_{K}=(\mathbf{I d}+0) \mathbf{A}_{J}+0=\mathbf{A}_{J}, \\
& \widehat{\mathbf{P}}_{J}\left(\mathbf{q}, \dot{\mathbf{q}}_{s}\right)=-\Lambda_{J} \mathbf{A}_{J}\left(\mathbf{I d}-(1-s) \mathbf{A}_{K}^{\dagger T} \mathbf{A}_{K}\right) \dot{\mathbf{q}}=s\left(-\Lambda_{J} \mathbf{A}_{J} \dot{\mathbf{q}}\right)=s \widehat{\mathbf{P}}_{J}(\mathbf{q}, \dot{\mathbf{q}}),
\end{aligned}
$$

we have,

$$
\forall j \in J: \mathbf{U}_{j}\left(\widehat{\mathbf{P}}_{J}\left(\mathbf{q}, \dot{\mathbf{q}}_{s}\right)\right)=\mathbf{U}_{j}\left(s \widehat{\mathbf{P}}_{J}(\mathbf{q}, \dot{\mathbf{q}})\right)
$$

Therefore

$$
\forall j \in J: \mathbf{U}_{j}\left(\widehat{\mathbf{P}}_{J}\left(\mathbf{q}, \dot{\mathbf{q}}_{s}\right)\right) \geq 0 \Leftrightarrow \mathbf{U}_{j}\left(\widehat{\mathbf{P}}_{J}(\mathbf{q}, \dot{\mathbf{q}})\right) \geq 0
$$

the conclusion of the Lemma follows directly. 


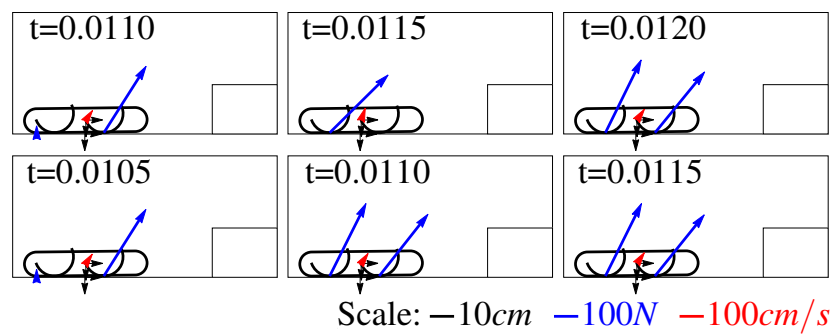

Figure 3: Keyframes around the impact of the second leg with the ground - note the difference in contact forces (blue arrows) which indicate which contacts are active. Top Row: Without pseudo-impulse $\left(\delta_{t}=0\right)$. Bottom Row: With pseudo-impulse $\left(\delta_{t}=0.03\right)$. The center top frame shows that the contact with the front leg is lost when the rear leg touches down (and therefore no contact force is possible), but the center bottom frame shows both contacts are maintained with the pseudo-impulse (and therefore both contacts can apply forces to the system). Note that there is a slight difference in touchdown time due to similar discrepancies around the time of the first leg touchdown. See also Figure 4.

Theorem 3. Assume the same conditions as in Lemma 10. If further there is a unique constraint $i \in I \backslash J$ such that,

$$
\mathbf{U}_{i}\left(\mathbf{A}_{J \cup\{i\}}^{\dagger}(\mathbf{q})\left(\Upsilon\left(\mathbf{q}, \dot{\mathbf{q}}_{0}\right)-\overline{\mathbf{C}}\left(\mathbf{q}, \dot{\mathbf{q}}_{0}\right)-\overline{\mathbf{N}}\left(\mathbf{q}, \dot{\mathbf{q}}_{0}\right)\right)\right)>0,
$$

(i.e., a constraint $i \in I$ is impinged upon by external forces but would be removed after impact with constraint $k$ if $\left.\delta_{t}=0\right)$ then for all values of the pseudo-impulse parameter $\delta_{t}>0$, there exists $\bar{s}>0$ such that with $\dot{\mathbf{q}}_{s}$ defined as in (60) we have,

$$
\forall s \in(0, \bar{s}): i \in \mathrm{CP}_{\mathrm{PIV}}\left(\mathbf{q}, \dot{\mathbf{q}}_{s}\right),
$$

that is, the pseudo-impulse prevents the liftoff of constraint i for all sufficiently small impacting speeds.

Proof. For all $s>0$ let $K(s):=\operatorname{CP} \operatorname{PIV}\left(\mathbf{q}, \dot{\mathbf{q}}_{s}\right)$. Recall from (59) that $\mathrm{CP}_{\mathrm{IV}}\left(\mathbf{q}, \dot{\mathbf{q}}_{s}\right) \subset \mathrm{CP}_{\mathrm{PIV}}\left(\mathbf{q}, \dot{\mathbf{q}}_{s}\right)$ for all $s \geq 0$. Thus although $K(s)$ may not be constant, we are only concerned with the asymptotic inclusion of additional constraints.

Note that,

$$
\lim _{s \rightarrow 0^{+}} \widehat{\mathbf{P}}_{K(s)}\left(\mathbf{q}, \dot{\mathbf{q}}_{s}\right)=\mathbf{0},
$$

and therefore for all values of the pseudo-impulse parameter $\delta_{t}>0$,

$$
\lim _{s \rightarrow 0^{+}} \mathbf{U}_{k}\left(\widehat{\mathbf{P}}_{K(s)}\left(\mathbf{q}, \dot{\mathbf{q}}_{s}\right)+\widetilde{\mathbf{P}}_{K(s)}\left(\mathbf{q}, \dot{\mathbf{q}}_{s}\right)\right)=\mathbf{U}_{k}\left(\widetilde{\mathbf{P}}_{K(s)}\left(\mathbf{q}, \dot{\mathbf{q}}_{s}\right)\right) .
$$

for all $k \in K(s)$. By assumption, constraint $i$ is the only constraint such that $\mathbf{U}_{i}(\widetilde{\mathbf{P}})>0$ and, by (58), is to be included in the solution. As no other constraints are added or removed, there must exist some $\bar{s}>0$ that ensures (67) holds.

While Theorem 3 is limited to only single constraints, the pseudo-impulse parameter can similarly prevent the impulsive liftoff of at least some constraints when there are multiple that satisfy (66), subject to the nature of the PIV complementarity problem.

\subsubsection{Pseudo-Impulse Example}

The pseudo-impulse can help to resolve certain Zeno executions, as shown in Section 3.5.2, but more importantly it reduces "chattering", or executions that involve many impulsive transitions that are qualitatively undesirable. As an example from the RHex leaping simulation of Figure 1 (see also the additional examples explored in Section 4.3), Figure 3 compares the state just before and after the rear leg touches down with and without the pseudo-impulse term. At that instant the calculated impulse (25) is $P_{l}=-1.47 \mathrm{Ns}$ (in the normal direction on the front leg). Even though the leg motor is applying maximum torque trying to keep the leg on the ground the small negative impulse causes the leg to separate, and then the motor torque quickly accelerates the leg back to the ground (with or without massless legs, as recall that even massless legs are assumed to have finite acceleration, thus the leg may return quickly but not instantly). With the pseudo-impulse this is balanced out by $P_{\delta, 1}=7.91 \mathrm{Ns}$, and the leg does not leave the ground (as 


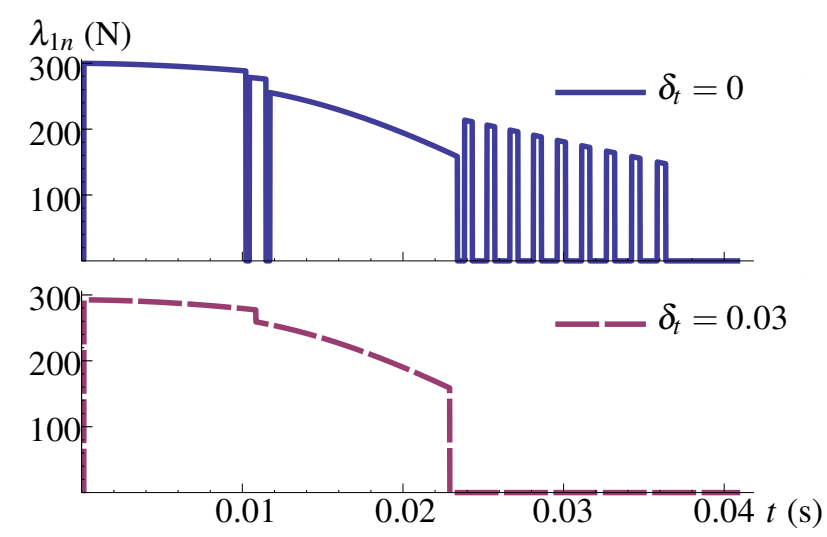

Figure 4: Comparison of the front leg normal direction ground reaction force for evaluations with and without the pseudo-impulse. See also Figure 3.

would be the case on the real robot in this configuration to within modeling precision). If the induced impulse were much larger then the desired result may be for the front leg to lift off the ground, while a much smaller impulse would clearly not break the front leg's contact. The $\delta_{t}$ term is in essence a tuning parameter that determines the threshold between a quasi-static regime (where contacts are maintained) and a dynamic regime (where impulses may break existing contacts).

Impulsively breaking contact at the wrong time is an even bigger problem when considering a full behavior and not just analyzing an individual impact event. As Figure 4 suggests, without a pseudo-impulse this impulsive liftoff can lead to chattering. In this case starting around $t=0.023$ the front leg lifts off but the continuous time forces return the leg to the ground after a short time. When the front leg impacts the ground, the rear leg then impulsively breaks contact, and a cyclic oscillation begins. This behavior is not quite a Zeno-execution, as the finite acceleration of the leg in the air results in only finitely many transitions in a finite time, however these transitions are still qualitatively undesirable.

\subsection{Friction}

While this paper is not focused on methods for modeling friction, including friction in some form is unavoidable (McGeer and Palmer 1989). Here, in order to advance our targeted conclusions guaranteeing the existence and uniqueness of a solution, we assume that,

Assumption $\mathbf{A 1 2}$ (Friction). All contact points with Coulomb friction are attached only to massless links. Contact points without friction are assumed to never resist sliding motion, and all contact points that are sliding have no kinetic coefficient of friction. No sliding-to-sticking transitions are considered.

The velocity constraint, $\mathbf{A}$, unilateral constraint cone, $\mathbf{U}$, and complementarity problems, $\mathrm{CP}$, are thus taken to include any active frictional constraints - see Section 2.1 or (Johnson and Koditschek 2013a, Assumptions C.3 and C.4) for details.

This restrictive frictional assumption ensures that during impact (i.e., in evaluating $\mathrm{CP}_{\mathrm{IV}}$, the impulse-velocity complementarity problem (50)) any conflict involving the frictional constraints can be resolved by simply removing that contact (the normal and tangential constraints) from the active set (see Section 1.3.5 for a summary of pathologies that arise when this assumption is relaxed). As a massless link, it can always rotate out of the way fast enough (as discussed above in Section 2.6).

In continuous time (i.e., in evaluating $\mathrm{CP}_{\mathrm{FA}}$, the force-acceleration complementarity problem (38)) this frictional assumption as applied to the RHex model states that the body has a low coefficient of friction and does not resist tangential forces while the legs' rubber feet have a high coefficient of friction and therefore typically do resist tangential forces. However it is known that even for contacts with infinite friction, allowing a sliding mode is sometimes required to find a consistent solution to the frictional force-acceleration complementarity problem (McGeer and Palmer 1989). Furthermore, a strict a priori assumption about friction is certainly not a good model for every situation - consider what happens when RHex's legs push against each other, as with the vertical leap described in Johnson and Koditschek (2013b) (see in particular Footnote 8 and the end of Section V.A). In order to model such a behavior the leg contact 


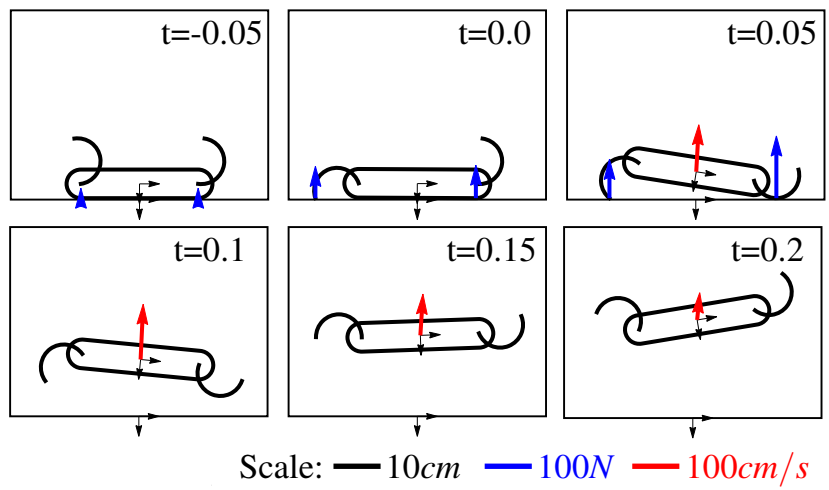

Figure 5: Keyframes from RHex simulation leaping vertically to a height of $37 \mathrm{~cm}$. Blue arrows show contact forces while the red arrow shows body velocity. The coefficient of friction is $\mu=0.8$ and the relative leg timing is $t_{2}=-0.06 \mathrm{~s}$.

points must be allowed to transition to sliding contact when the contact forces reach the friction cone in the tangential direction, $\mathbf{U}_{k}(\lambda) \geq 0$ (Johnson and Koditschek 2013a, Eqn. 4), as with the liftoff condition in (35)-(36). Allowing this transition enables for example the simulation of the vertical leap shown in Figure 5 or the leap onto a ledge shown in Figure 10, which each use a (hand tuned) value of $\mu=0.8$. The forward leap of Figure 1 still requires this transition, for much the same reason as in (McGeer and Palmer 1989), but uses the relatively high values of $\mu=1.8$ for the front leg and $\mu=2.5$ for the rear. After transition to sliding the kinetic coefficient of friction is taken as $\mu_{k}=0$ (as with the frictionless body contact points) so that the jamming problems discussed in Section 1.3.5 are again avoided.

The transition from sliding to sticking is much trickier. A sliding constraint sticks when the tangential velocity drops to zero and the resulting contact forces lie within the friction cone, i.e. contact $k \in \mathcal{K}_{t}$ is to be added if and only if its corresponding normal constraint is active $(\alpha(k) \in I)$ and,

$$
\mathbf{A}_{k} \dot{\mathbf{q}}=0 \wedge \mathbf{U}_{k}\left(\lambda_{I \cup\{k\}}\right) \succeq 0 .
$$

However this additional condition complicates the force-acceleration complementarity problem and furthermore is not needed to model any of the leaping behaviors in Johnson and Koditschek (2013b), our motivating scenario. Therefore for the purposes of this paper we do not consider such slip-to-stick transitions. This limitation prevents, e.g., the modeling of a leg that slides upon contact with the ground but gains traction later using static friction.

\section{Hybrid Dynamics}

In this section we first define a general $C^{r}$ hybrid dynamical system (Section 3.1) that is then instantiated as the main object of study for this paper, the self-manipulation hybrid dynamical system (Section 3.2). Section 3.3 establishes that this system is indeed a $C^{r}$ hybrid dynamical system and Section 3.4 further shows its internal consistency. Finally, Section 3.5 shows that Zeno executions of the system accumulate and that the pseudo-impulse truncates certain Zeno executions.

\section{1 $C^{r}$ Hybrid Dynamical System}

In the following definitions we make use of the natural (disjoint-union) topology on the hybrid state space, consisting of a collection of manifolds with corners (Joyce 2012, Def. 2.1); see Appendix D or (Burden et al. 2015b, Sec. II) for more details. The hybrid system notation introduced in this section is summarized in Table 2.

Definition 2. $A C^{r}$ hybrid dynamical system, $r \in \mathbb{N} \cup\{\infty, \omega\}$, is a tuple $\mathcal{H}:=(\mathcal{J}, \Gamma, \mathcal{D}, \mathcal{F}, \mathcal{G}, \mathcal{R})$, where the constituent parts are defined as:

1. $\mathcal{J}:=\{I, J, \ldots, K\} \subset \mathbb{N}$ is the finite set of discrete modes;

2. $\Gamma \subset \mathcal{J} \times \mathcal{J}$ is the set of discrete transitions, forming a directed graph structure over $\mathcal{J}$; 


\begin{tabular}{ll}
\hline \hline $\mathcal{D}:=\bigsqcup_{I \in \mathcal{J}} D_{I}$ & Hybrid system domain (Def. 2) \\
$\mathcal{F}: \mathcal{D} \rightarrow T \mathcal{D}, F_{I}:=\left.\mathcal{F}\right|_{D_{I}}$ & Vector field (Def. 2) \\
$\mathcal{G}:=\coprod_{(I, J)} G_{I, J}, G_{I, J} \subset D_{I}$ & Guard set (Def. 2) \\
$\mathcal{H}:=(\mathcal{J}, \Gamma, \mathcal{D}, \mathcal{F}, \mathcal{G}, \mathcal{R})$ & Hybrid dynamical system (Def. 2) \\
$\mathcal{J} \subset \mathbb{N}$ & Discrete indexing set (Def. 2) \\
$\mathcal{R}: \mathcal{G} \rightarrow \mathcal{D}, R_{I, J}:=\left.\mathcal{R}\right|_{G_{I, J}}$ & Reset map (Def. 2) \\
$\mathcal{T}:=\bigsqcup_{i} T_{i}, T_{i} \subset \mathbb{R}$ & Hybrid time domain (Def. 3) \\
$\Gamma \subset \mathcal{J} \times \mathcal{J}$ & Set of discrete transitions (Def. 2) \\
$\sigma(\chi) \in \mathcal{J}^{N}$ & Contact word of length $N$ (Def. 4) \\
$\chi: \mathcal{T} \rightarrow \mathcal{D}$ & Execution of the system (Def. 4) \\
\hline
\end{tabular}

Table 2: Hybrid system and execution symbols, with definition of introduction marked. See also Table 1 for symbols introduced in Section 2.

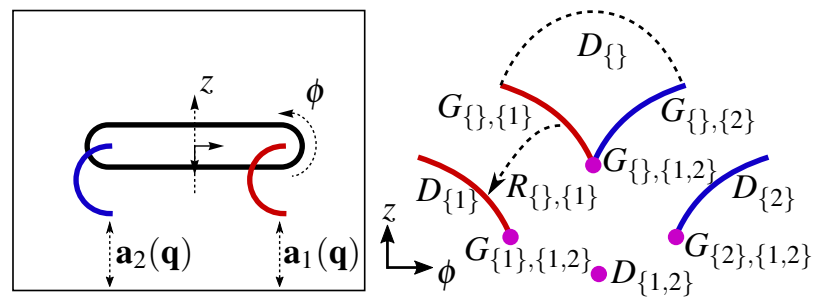

Figure 6: Illustration of elements from the $C^{r}$ hybrid dynamical system (Definition 2) for the RHex model. Note that this is a 5-dimensional model $(\operatorname{dim} \mathcal{Q}=5)$, so we cannot faithfully represent the domains and guards on the printed page; instead, we illustrate a two-dimensional slice of height $z$ and body pitch $\phi$. From the unconstrained mode $D_{\{\}}$there are three possible discrete transitions corresponding to touchdown of the front leg $\left(G_{\{\},\{1\}} \subset\left\{(\mathbf{q}, \dot{\mathbf{q}}) \in T \mathcal{Q}: \mathbf{a}_{1}(\mathbf{q})=0\right\}\right)$, rear leg $\left(\mathbf{a}_{2}(\mathbf{q})=0\right)$, or simultaneous touchdown of both legs. We annotate the reset map $R_{\{\},\{1\}}$ corresponding to front leg touchdown, but emphasize that there are corresponding maps defined over all the guards $G_{I, J}$. Each constrained mode also generally contains liftoff guards (e.g. $G_{\{1\},\{\}} \subset D_{\{1\}}$ ); these are not illustrated.

3. $\mathcal{D}:=\amalg_{I \in \mathcal{J}} D_{I}$ is the collection of domains, where $D_{I}$ is a $C^{r}$ manifold with corners;

4. $\mathcal{F}: \mathcal{D} \rightarrow T \mathcal{D}$ is a $C^{r}$ hybrid map that restricts to a vector field $F_{I}:=\left.\mathcal{F}\right|_{D_{I}}$ for each $I \in \mathcal{J}$;

5. $\mathcal{G}:=\amalg_{(I, J) \in \Gamma} G_{I, J}$ is the collection of guards, where $G_{I, J} \subset D_{I}$ for each $(I, J) \in \Gamma$;

6. $\mathcal{R}: \mathcal{G} \rightarrow \mathcal{D}$ is a continuous map called the reset that restricts as $R_{I, J}:=\left.\mathcal{R}\right|_{G_{I, J}}: G_{I, J} \rightarrow D_{J}$ for each $(I, J) \in \Gamma$.

Before we proceed, we make a few clarifying comments about this definition. While $\Gamma$ is a directed graph it is not generally a tree (i.e., $(I, J)$ and $(J, I)$ may both be members). When we write $\mathcal{G}:=\coprod_{(I, J) \in \Gamma} G_{I, J}$ where $G_{I, J} \subset D_{I}$ for each $(I, J) \in \Gamma$, we are simultaneously specifying that (i) each $G_{I, J}$ is an arbitrary subset of $D_{I}$ and (ii) $\mathcal{G}$ is the finite disjoint union of these subsets. The domain $\mathcal{D}$ should be regarded as a $C^{r}$ hybrid manifold as described in Appendix D since each $D_{I}$ is a $C^{r}$ manifold with corners. In contrast the guard $\mathcal{G}$ does not generally possess a smooth structure since each $G_{I, J} \subset D_{I}$ is not even required to be a topological manifold. We say that $\mathcal{H}$ has disjoint guards if $G_{I, J} \cap G_{I, L}=\varnothing$ for each pair $(I, J),(I, L) \in \Gamma$ such that $J \neq L$. An illustration of some of the elements of a $C^{r}$ hybrid dynamical system is shown in Figure 6.

Roughly speaking, an execution of a hybrid dynamical system is set in motion from an initial condition in $\mathcal{D}$ by following the continuous-time dynamics determined by the vector field $\mathcal{F}$ until the trajectory reaches the guard $\mathcal{G}$, at which point the reset map $\mathcal{R}$ is applied to obtain a new initial condition. We formalize this using the notion of a hybrid time domain.

Definition 3. A hybrid time domain is a disjoint union of intervals $\mathcal{T}:=\coprod_{i=1}^{N} T_{i}$ such that: 
1. $N \in \mathbb{N} \cup\{\infty\}$;

2. $T_{i} \subset \mathbb{R}$ is a closed interval for all $i<N$, and if $N<\infty$ then $T_{N} \subset \mathbb{R}$ is also a closed interval;

3. $T_{i} \cap T_{i+1}$ is nonempty and consists of a single element for all $i<N$.

Note that an interval may be degenerate, i.e. $T_{i}=\left\{t_{i}\right\}$. We define $\sup \mathcal{T}:=\sup \bigcup_{i=1}^{N} T_{i}$.

This definition is essentially equivalent to the hybrid time trajectory (Lygeros et al. 2003), the hybrid time set (Collins 2004), and the hybrid time domain (Goebel and Teel 2006), and enables us to formalize the conceptual description of the domain of a hybrid execution from Back et al. (1993) as being divided into contiguous epochs separated by events where the reset map is applied at an instant referred to as an event time. Furthermore, this definition has two appealing features. First, an execution (defined below) becomes a smooth (hybrid) map defined from a hybrid time domain $\mathcal{T}$ into the continuous state space $\mathcal{D}$ of the hybrid system, avoiding the use of set-valued maps or cumbersome left- or right-handed limits; see Appendix D for the definition of smoothness for hybrid maps. Second, it treats the model of time in the same class of mathematical objects as the model for the state space, namely, a disjoint union of smooth manifolds with corners. Note that under this definition a transition time $t_{i} \in T_{i} \cap T_{i+1}$ appears in two consecutive components of the time domain $T_{i}$ and $T_{i+1}$, allowing the flow on each interval to include both endpoints. Also note that this allows for two transitions (or more) to occur at the same instant in time, e.g. it is possible that $T_{i}=\left\{t_{i}\right\}, T_{i-1} \cap T_{i} \cap T_{i+1} \cap \ldots=\left\{t_{i}\right\}$; the middle portion of the trajectory would have been excised from a left- or right-handed definition of execution, or potentially muddled with the surrounding trajectory portions in a set-valued definition.

Definition 4. An execution of a hybrid dynamical system $\mathcal{H}=(\mathcal{J}, \Gamma, \mathcal{D}, \mathcal{F}, \mathcal{G}, \mathcal{R})$ is a smooth map $\chi: \mathcal{T} \rightarrow \mathcal{D}$ over a hybrid time domain $\mathcal{T}=\bigsqcup_{i=1}^{N} T_{i}$ satisfying:

1. $\forall i \in \mathbb{N}, i \leq N$ : if $T_{i}$ is not a degenerate interval then $\left.\frac{d}{d t} \chi\right|_{T_{i}}(t)=\mathcal{F}(\chi(t))$ for all $t \in T_{i}$;

2. $\forall i<N$ : for $\left\{t_{i}\right\}=T_{i} \cap T_{i+1}$ (the event times), we have $\left.\chi\right|_{T_{i}}\left(t_{i}\right) \in \mathcal{G}, \mathcal{R}\left(\left.\chi\right|_{T_{i}}\left(t_{i}\right)\right)=\left.\chi\right|_{T_{i+1}}\left(t_{i}\right)$, and for all $s \in T_{i} \backslash\left\{t_{i}\right\}$ we have $\left.\chi\right|_{T_{i}}(s) \notin \mathcal{G}$.

The execution has an associated word denoted by $\sigma(\chi)=\left\{J_{i}\right\}_{i=1}^{N} \in \mathcal{J}^{N}$ that specifies the sequence of discrete modes encountered by the execution: $\left.\chi\right|_{T_{i}} \subset D_{J_{i}}$ for all $i \in \mathbb{N}, i \leq N$. An execution $\chi: \mathcal{T} \rightarrow \mathcal{D}$ is maximal if it cannot be extended to an execution over a longer hybrid time domain. We say ${ }^{19}$ that $\mathcal{H}$ is: deterministic if for every initial condition $x \in \mathcal{D}$ there exists a unique maximal execution $\chi: \mathcal{T} \rightarrow \mathcal{D}$ such that $\left.\chi\right|_{T_{1}}(0)=x$; non-blocking if for every initial condition $x \in \mathcal{D}$ and any maximal execution $\chi: \mathcal{T} \rightarrow \mathcal{D}$ with $\left.\chi\right|_{T_{1}}(0)=x$, then with $\mathcal{T}=\bigsqcup_{i=1}^{N} T_{i}$ either $N=\infty$ or $N<\infty$ and $T_{N}=\left[t_{N}, \infty\right)$.

The contact word, $\sigma(\chi)$, also called the contact motion plan, is useful for comparing and reasoning about different executions of the hybrid system (Xiao and Ji 2001).

\subsection{The Self-Manipulation System}

While the previous hybrid system specification is very general, it is useful to instantiate it for a model of a physical system. This section defines the self-manipulation system (Johnson and Koditschek 2013a) (and by the analogy of that paper, equivalently a manipulation system (Murray et al. 1994), as summarized in Section 2.2), where the discrete mode, $I \subset \mathcal{K}$, corresponds to the active contact mode.

Definition 5. A self-manipulation hybrid system is a $C^{r}$ hybrid dynamical system, $\mathcal{H}_{s}=(\mathcal{J}, \Gamma, \mathcal{D}, \mathcal{F}, \mathcal{G}, \mathcal{R})$, defined as follows,

\subsubsection{Discrete Modes}

The set of modes, all physically permissible combinations of contact constraints, is given by,

$$
\mathcal{J}=\left\{I \in 2^{\mathcal{K}}: \mathbf{a}_{I_{n}}^{-1}(0) \neq \varnothing \wedge \alpha\left(I_{t}\right) \subset I\right\},
$$

\footnotetext{
${ }^{19}$ Following Lygeros et al. (2003).
} 
that is there are two requirements: 1) there must exist some point, $\mathbf{q} \in \mathcal{Q}$, such that these normal contact constraints are active, $\mathbf{a}_{I_{n}}(\mathbf{q})=0$, and 2) no tangential constraint is included whose corresponding normal constraint is not also included, i.e. $\nexists i \in I_{t}: \alpha(i) \notin I$.

\subsubsection{Edges}

The set of edges is made up of any pair of modes whose union is also a mode - in other words, sets arising from the intersection of the two base sets that satisfy respectively the two sets of normal constraints,

$$
\Gamma=\{(I, J) \in \mathcal{J} \times \mathcal{J}: I \neq J, I \cup J \in \mathcal{J}\} .
$$

This set of edges can be further restricted based on the guards, defined below, as there are some transitions $(I, J) \in \Gamma$ where no points in $D_{I}$ satisfy the requirements of the guard, i.e. $G_{I, J}=\varnothing$. In that case we reduce the edge set further,

$$
\tilde{\Gamma}=\left\{(I, J) \in \Gamma: G_{I, J} \neq \varnothing\right\} .
$$

\subsubsection{Domains}

The domain associated with a contact mode $I \in \mathcal{J}$ is the subset of the ambient tangent bundle $T \mathcal{Q}$ that satisfies the normal non-penetration and tangential non-sliding constraints,

$$
D_{I}=\left\{(\mathbf{q}, \dot{\mathbf{q}}) \in T \mathcal{Q}: \mathbf{a}_{I_{n}}(\mathbf{q})=0, \mathbf{a}_{\mathcal{K}_{n}}(\mathbf{q}) \geq 0, \mathbf{A}_{I}(\mathbf{q}) \dot{\mathbf{q}}=0\right\}
$$

where recall that $\mathcal{Q}:=\Theta \times S E(\mathrm{~d})$ is the joint space combined with the position space of the body.

\subsubsection{Flows}

The vector field on each domain is based on the self-manipulation dynamics for $\ddot{\mathbf{q}}$, as in (12) and (Johnson and Koditschek 2013a, Eqn. 33, 72), are,

$$
F_{I}(\mathbf{q}, \dot{\mathbf{q}})=\left[\dot{\mathbf{q}}, \quad \overline{\mathbf{M}}^{\dagger}(\Upsilon-\overline{\mathbf{C}} \dot{\mathbf{q}}-\overline{\mathbf{N}})-\mathbf{A}_{I}^{\dagger T} \dot{\mathbf{A}}_{l} \dot{\mathbf{q}}\right] .
$$

for the coordinates in $\mathcal{Q}_{I}$, and recall from Assumption A6 (unconstrained massless limbs) that the coordinates associated with unconstrained massless limbs lie in the subspace $\tilde{\mathcal{Q}}_{I}$ and evolve according to the vector field $\tilde{F}$, such that the combined vector field is complete over all of $T \mathcal{Q}$. The control input $\tau \in T^{*} \Theta$ that appears in $\Upsilon$ is prescribed by a $C^{r}$ function of state $\tau \in C^{r}\left(T \mathcal{Q}, T^{*} \mathcal{Q}\right.$ ) (for example a fixed-voltage motor model $\tau_{i}=\kappa_{P} \kappa_{G}\left(1-\kappa_{G} \dot{\theta}_{i}\right)$ (Johnson and Koditschek 2013a, Sec. IV.C.4)).

\subsubsection{Guards}

We find it convenient to construct the guard set, for mode $I, G_{I} \subset D_{I}$, as a union of subsets indexed by its "outgoing" edges, $(I, J) \in \Gamma$, using the touchdown predicate (22) and the complementarity problem predicates (58) and (38) specified as ${ }^{20}$,

$$
\begin{aligned}
G_{I, J}=\left\{\mathbf{T q} \in D_{I}: \operatorname{NTD}(\mathbf{T q})\right. & \Rightarrow \operatorname{PIV}(J, \mathbf{T q}), \\
\neg \operatorname{NTD}(\mathbf{T q}) & \Rightarrow \operatorname{FA}(J, \mathbf{T q})\} .
\end{aligned}
$$

Conceptually, the component of the guard for mode $I$ associated with edge $(I, J)$ consists of any base states, $\mathbf{q}$, at which any new touchdown event can occur from mode $I$ into mode $J$, according to the NTD predicate, (22), subject to PIV complementarity, (58). An additional condition on the base and tangent states, $\mathbf{T q}$, is that if no new contacts are touching down ("liftoff"), then FA complementarity holds (38).

\footnotetext{
${ }^{20}$ Note that the requirement that $(I, J) \in \Gamma$ and therefore $J \in \mathcal{J}$ ensures that all tangential constraints in the new contact condition must have a matching normal constraint also (or trending so). Furthermore, as in (73), note that only some of these outgoing edges make a non-empty contribution, $G_{I, J}$, to this union.
} 
The outlet set in domain $I$, defined as $G_{I}:=\cup_{J} G_{I, J}$, is,

$$
G_{I}=\left\{\mathbf{T q} \in D_{I}:\left(\bigvee_{k \in \mathcal{K}_{n} \backslash I} \mathbf{a}_{k}(\mathbf{q}) \preceq 0\right) \bigvee\left(\bigvee_{i \in I} \mathbf{U}_{i}\left(\lambda_{I}\right) \prec 0\right)\right\} .
$$

The outlet set is used in the proof of Theorem 7, but more importantly provides a computationally expedient method of simulating an execution: first check if $\mathbf{T q} \in G_{I}$, then determine the subsequent mode afterwards,

$$
J=\left\{\begin{array}{lr}
\mathrm{CP}_{\mathrm{PIV}}(\mathbf{T q}), & \mathrm{NTD}(\mathbf{T q}) \\
\mathrm{CP}_{\mathrm{FA}}(\mathbf{T q}), & \neg \mathrm{NTD}(\mathbf{T q}) .
\end{array}\right.
$$

\subsubsection{Reset Maps}

The reset map associated with edge $(I, J) \in \Gamma$ (taking its domain exactly on $G_{I, J}$, defined above) is,

$$
R_{I, J}(\mathbf{q}, \dot{\mathbf{q}})=\left[\begin{array}{ll}
\mathbf{q}, & \dot{\mathbf{q}}-\Delta \dot{\mathbf{q}}_{J}
\end{array}\right]=\left[\begin{array}{ll}
\mathbf{q}, & \dot{\mathbf{q}}-\mathbf{A}_{J}^{\dagger T} \mathbf{A}_{J} \dot{\mathbf{q}}
\end{array}\right] .
$$

Note that for takeoff events, $J_{n} \subseteq I_{n}$, the prior velocity already agrees with the new contact mode and therefore the impact map has no effect.

\subsection{The Self-Manipulation System is a Hybrid System}

This section shows that,

Theorem 4. The self-manipulation system (Def. 5) is a $C^{\omega}$ hybrid dynamical system (Def. 2).

Proof. Definition 2 has a number of requirements and so this proof is broken up into the constituent parts and show that each component of Definition 5 is compatible with the requirements.

1. $\mathcal{J}$ in (71) is a finite set, the only requirement on $\mathcal{J}$.

2. $\Gamma$ in (72) is a subset of $\mathcal{J} \times \mathcal{J}$ by construction, and $\tilde{\Gamma}$ in (73) is a subset of $\Gamma$.

3. By Assumption A2 (simple constraints), for all $I \in \mathcal{J}$ the maps $\mathbf{a}_{I_{n}} \in C^{\omega}\left(\mathcal{Q}, \mathcal{C}_{I_{n}}\right)$ and $\mathbf{A}_{I} \in C^{\omega}\left(T \mathcal{Q}, T \mathcal{C}_{I}\right)$ are constant rank, and therefore each $D_{I} \subset T \mathcal{Q}$, as defined by these functions in (74) is a closed $C^{\omega}$ manifold with corners (Lee 2012, Thm. 5.12), (Joyce 2012, Def. 2.1). The Nash Embedding Theorem (Nash 1966) states that $\mathcal{Q}$ can be embedded analytically in Euclidean space of sufficiently high dimension; this embedding therefore restricts to an embedding of the submanifold $D_{I}$.

4. For all $(\mathbf{q}, \dot{\mathbf{q}}) \in D_{I}, F_{I}(\mathbf{q}, \dot{\mathbf{q}}) \in T_{(\mathbf{q}, \dot{\mathbf{q}})} D_{I}$ for $F_{I}$ given in (75) (based on (12), (Johnson and Koditschek 2013a, Eqn. 33), which enforce the equality constraints of the definition of the domain, (74), and therefore lie within $\left.T D_{I}\right)$ and hence we may write $F_{I} \in C^{\omega}\left(D_{I}, T D_{I}\right)$.

5. $G_{I, J}$ in (76) is a subset of $D_{I}$ by construction.

6. For the reset map in (80), $\operatorname{Im} R_{I, J}\left(G_{I, J}\right) \subset D_{J}$, as the domain $D_{J}$ has three requirements (74):

1) $\mathbf{a}_{J_{n}}(\mathbf{q})=0$, i.e. any normal constraints are touching the surface indicated. For pre-existing constraints, $\{i$ : $\left.i \in I_{n} \cap J_{n}\right\}$, this requirement is already guaranteed, i.e. $\mathbf{q} \in G_{I, J} \subset D_{I} \Rightarrow \mathbf{a}_{I_{n}}=0$, and the reset map does not alter the base coordinates q. New normal constraints in mode $J,\left\{j: j \in J_{n} \backslash I_{n}\right\}$, satisfy this requirement by the touchdown predicate in the guard (76) (and (21)), where $\operatorname{TD}(j, \mathbf{T q})$ is true only when $\mathbf{a}_{j}(\mathbf{q})=0$.

2) $\mathbf{a}_{\mathcal{K}_{n}}(\mathbf{q}) \geq 0$, i.e. all base constraints are non-negative. Again since the reset map does not alter the base coordinates $\mathbf{q}$, then $\mathbf{q} \in G_{I, J} \subset D_{I} \Rightarrow \mathbf{a} \mathcal{K}_{n} \geq 0$.

3) $\mathbf{A}_{J}(\mathbf{q}) \dot{\mathbf{q}}=0$, i.e. any velocity in constrained directions is zero, but this is guaranteed by the reset map as $\mathbf{A}_{J} \dot{\mathbf{q}}^{+}=\mathbf{A}_{J} \dot{\mathbf{q}}^{-}-\mathbf{A}_{J} \mathbf{A}_{J}^{\dagger T} \mathbf{A}_{J} \dot{\mathbf{q}}^{-}=0$. Therefore, as claimed, the image of the reset map (80) lies within $D_{J}$.

\subsection{Consistency Properties}

This section establishes several additional properties of the self-manipulation system that are of practical importance, Theorems 5-9, which we shall for convenience collectively call consistency properties.

Theorem 5. The self-manipulation system (Def. 5) has disjoint guards. 
Proof. The disjointedness of the guards follows directly from the assumption of uniqueness of solution for the constituent complementarity problems. Define the liftoff predicate where no contact is touching down,

$$
\mathrm{LO}(I, \mathbf{T q}):=\neg \mathrm{NTD}(\mathbf{T} \mathbf{q}) \wedge\left(\bigvee_{k \in \mathcal{I}} \mathbf{U}_{k}\left(\lambda_{I}\right) \prec 0\right),
$$

and so by a refinement of the complementary block of the partition defined on the right hand side of (79),

$$
\mathrm{CP}_{G}(I, \mathbf{T q})= \begin{cases}\mathrm{CP}_{\mathrm{PIV}}(\mathbf{T q}), & \mathrm{NTD}(\mathbf{T q}) \\ \mathrm{CP}_{\mathrm{FA}}(\mathbf{T q}), & \mathrm{LO}(I, \mathbf{T q}) \\ I, & \text { otherwise },\end{cases}
$$

is equal to either the unique mode $J$ for which $\mathbf{T q} \in G_{I, J}$, or simply $I$ if the state is not in a guard, $\mathbf{T q} \notin G_{I, J} \forall(I, J) \in$ $\Gamma$.

In their most general formulation, hybrid dynamical systems can accept executions that terminate before infinite time (continuous or discrete) has elapsed, or accept multiple distinct executions from the same initial condition. This behavior is undesirable in practice, and inconsistent with our experience on real manipulation and self-manipulation systems. Necessary and sufficient conditions (Lygeros et al. 2003, Lems. III.1, III.2) have been formulated that ensure a system is deterministic and non-blocking. Since these conditions are applicable to a general class of hybrid dynamical systems, they can be difficult to verify directly for particular classes of models. No previous authors have established that these conditions hold for any broad class of hybrid system models for Lagrangian dynamics subject to multiple unilateral constraints, much less with the particular structure of the self-manipulation system. The conditions in (Johansson et al. 1999, Lem. 1 \& 2) come closest, as they would apply to an instance of a self-manipulation system exhibiting only a single constraint.

To serve the needs of the present paper, we introduce an extension of the line of reasoning in Lygeros et al. (2003) establishing that the self-manipulation system, Definition 5, is indeed deterministic and non-blocking, Definition 4, in the presence of an arbitrary number of unilateral constraints.

Theorem 6. The self-manipulation system is deterministic.

Proof. Assumption A7 (Lagrangian dynamics) imposes a partial flow on each component $D_{I}$, hence continuous trajectories are unique and nondeterminism could only be introduced through a reset. But the definition of execution, Def. 4, implies that a discrete transition occurs at $\mathbf{T q} \in D_{I}$ if and only if there exists $J \in \mathcal{J} \backslash\{I\}$ such that $\mathbf{T q} \in G_{I, J}$. Since the guards are disjoint by Theorem 5, there is at most one guard containing Tq. The execution continues from $R_{I, J}(\mathbf{T q}) \in D_{J}$.

The non-blocking property is a bit more subtle as the self-manipulation systems escape some of the structure required to handle the more general class of systems addressed in Lygeros et al. (2003) and used there to establish conditions for non-blocking. For self-manipulation hybrid systems the non-blocking property arises from the discrete logic and continuous dynamics in an essential manner that we now rehearse informally in preparation for the statement and proof of Theorem 7. The guard, $G_{I}$, intersects the corresponding domain, $D_{I}$, both on the boundary of the domain (to handle impact on an erstwhile inactive constraint) as well as in the interior of the domain (to handle a sign change on some active constraint's contact force). An execution might be blocked by conventional finite escape, i.e., if the continuous flow brings some initial state to the boundary of the domain at a point in the complement of the guard in finite time. Alternatively, it might be blocked by hybrid ambiguity, i.e., if the continuous flow brings some initial state to some point that is in the complement of the guard but still lies on the boundary of the guard, for this would violate the semantics of execution that restricts continuous flow to closed intervals (formally, Def. 4 requires a minimum not merely an infinum - time of entry into a guard). In the following proof we preclude both cases by showing that the guard contains all points reached by the continuous flow that lie in the boundary of the domain or the interior of the domain but the boundary of the guard.

Theorem 7. The self-manipulation system is non-blocking.

Proof. Recall from part 6 of the proof of Theorem 4 that the image of the guard set under the reset map is within the domain (and thus the discrete transition is never blocking), and from Assumption A7 (Lagrangian dynamics) that 
the flow is forward complete over $T \mathcal{Q}$. Therefore we need only check that the flow only reachs the boundary of the domain or the boundary of the guard within the domain interior at a point which is included in the guard.

Recall from (74) the definition of $D_{I}$, and note that it is a subset,

$$
D_{I} \subset\left\{\mathbf{T q} \in \mathcal{Q}: \bigwedge_{k \in \mathcal{K}_{n} \backslash I} \mathbf{a}_{k}(\mathbf{q}) \geq 0\right\},
$$

where furthermore,

$$
\left(\bigwedge_{i \in I_{n}} \mathbf{a}_{i}(\mathbf{q})=0\right) \wedge\left(\bigwedge_{i \in I} \mathbf{A}_{I}(\mathbf{q}) \dot{\mathbf{q}}=0\right) .
$$

Note that the constraints in (84) are invariant under the flow of (12), whence under the completeness assumption (Assumption A7) it is only possible to flow out of $D_{I}$ in forward time by violating one of the inequality conditions of (83).

Recall from (78) the union of all guards, $G_{I}$, and then using Lemma 1 note that the closure of the union of all guards is,

$$
\bar{G}_{I}=\left\{\mathbf{T q} \in D_{I}:\left(\bigvee_{k \in \mathcal{K} \backslash I} \mathbf{a}_{k}(\mathbf{q}) \leq 0\right) \bigvee\left(\bigvee_{i \in I} \mathbf{U}_{i}\left(\lambda_{I}\right) \leq 0\right)\right\}
$$

Now consider an arbitrary point in the domain, Tq $\in D_{I}$. If $\mu(\mathbf{T q})>0$ for all $\mu \in\left\{\mathbf{a}_{k}\right\}_{k \in \mathcal{K} \backslash I} \cup\left\{\mathbf{U}_{i}\left(\lambda_{I}\right)\right\}_{i \in I}$ then Tq is on the interior of $D_{I} \backslash G_{I}$ and it is possible to flow for positive time while remaining in the domain $D_{I}$ and not reach a guard $G_{I}$. Otherwise there exists $k \in \mathcal{K} \backslash I$ such that $\mathbf{a}_{k}(\mathbf{T q})=0$ (i.e., the state has reached the boundary of the domain) or there exists $i \in I$ such that $\mathbf{U}_{i}\left(\lambda_{I}(\mathbf{T q})\right) \leq 0$ (i.e., a sign change on some active constraint's contact force). We now consider the two (mutually exclusive) possibilities concerning whether a contact condition or an active force is trending negative:

1. $\mathbf{a}_{k}(\mathbf{T q}) \succ 0$ for all $k \in \mathcal{K} \backslash I$ or $\mathbf{U}_{i}\left(\lambda_{I}\right) \succeq 0$ for all $i \in I$;

2. there exists $k \in \mathcal{K} \backslash I$ such that $\mathbf{a}_{k}(\mathbf{T q}) \preceq 0$ or there exists $i \in I$ such that $\mathbf{U}_{i}\left(\lambda_{I}(\mathbf{T q})\right) \prec 0$.

In case 1), when there is neither a negative trending contact nor active force, then it is possible to flow for positive time in the domain without intersecting any guard or leaving the domain (Lemma 3); this provides the unique extension to the execution. The contrary case 2) is just the situation the hybrid system's logic is designed to flag: i.e., Tq is in a guard, (78), so the application of the reset map provides the unique extension to the execution.

Therefore every initial condition $\mathbf{T q} \in D$ yields a unique execution defined over a hybrid time domain that spans infinite time (continuous or discrete), whence the self-manipulation system is non-blocking.

The self-manipulation hybrid system may undergo multiple hybrid transitions in succession at the same time $t$, as there is no "dwell time" requirement to continue after reset under the continuous dynamics for any minimum amount of time. Therefore it is important to bound the number of such multiple transitions to ensure that the continuous execution eventually continues over an open interval of time. Here, Theorem 8 relates the image of the reset map to the guard sets to show that continuous execution continues after at most two successive hybrid transitions.

As a simple example, consider the self-manipulation system model consisting of a point mass in a gravitational field that points away from a constraint surface (i.e., a ball under a ceiling). If the mass is initialized with a velocity that causes it to impact the constraint surface, it transitions first to the constrained (ceiling) contact mode through an impact that ensures zero relative velocity. After spending zero time in the constrained mode, and therefore at the same continuous time, the system transitions again back to the unconstrained mode as the mass succumbs to gravity. The execution continues in the unconstrained mode as the mass accelerates away from the ceiling. In the self-manipulation system these are treated as separate transitions. At the expense of a small amount of additional bookkeeping in the definition of execution, we dramatically simplify the specification of the reset map (in this example, eliminating the need for a reset map from the unconstrained mode to the same unconstrained mode consisting of impulses from a constraint not present in either the original or destination mode).

Theorem 8. An execution of a self-manipulation hybrid system without massless limbs may undergo no more than two hybrid transitions at a single time $t$. 
Proof. The guards are partitioned into two types by the new touchdown predicate, NTD(Tq), (22), into touchdown and liftoff (non-touchdown) components. It suffices to show simply the reset map (i) takes states that are in touchdown guards to either non-guard states, or states in a liftoff guard, and that (ii) the reset map always takes states in the liftoff guard to non-guard states.

To show (i), note that for all points $\mathbf{T q}$ in a touchdown guard, (76), the impulse-velocity complementarity ensures that all constraints $k$ that are touching but are not in the outgoing contact mode $J$ have a separating velocity, $\mathbf{A}_{k} \dot{\mathbf{q}}^{+}>0$, after the application of the reset map, if such a velocity is well defined (52) (as in Theorem 2). Therefore all constraints $k$ not in $J$ must either not be touching $\left(\mathbf{a}_{k}\left(\mathbf{T} \mathbf{q}^{+}\right)>0\right)$ or have a separating velocity $\left(\mathbf{A}_{k} \dot{\mathbf{q}}^{+}>0\right)$, but therefore cannot satisfy the touchdown predicate, $\operatorname{TD}\left(k, \mathbf{T q}^{+}\right),(21)$, and so $\operatorname{NTD}\left(\mathbf{T q}^{+}\right)$is false and the state after the reset map is either not in a guard or is in a liftoff guard.

To show (ii), note that for all points $\mathbf{T q}$ in a liftoff guard, no contacts are touching down, i.e. $\forall k \in \mathcal{K}_{n}, \operatorname{TD}(k, \mathbf{T q})$ is false. The force-acceleration complementarity problem that defines these guards, $\mathrm{FA}(J, \mathbf{T q}),(38)$, does not depend on the active mode, $I$. Furthermore, the reset map $R_{I, J}(\mathbf{T q})$ is simply identity, and so the state remains the same after this transition, $\mathbf{T q}=R_{I, J}(\mathbf{T q})$. Therefore $\mathbf{T q} \in G_{I, J}$ such that $\operatorname{NTD}(\mathbf{T q})$ is false and $\mathrm{FA}(J, \mathbf{T q})$ is true implies that $\operatorname{NTD}\left(R_{I, J}(\mathbf{T q})\right)$ is also false and that $\mathrm{FA}\left(J, R_{I, J}(\mathbf{T q})\right)$ is still the correct solution, and the state after the reset map is not in any guard.

Therefore at any given time $t$, the system can undergo at most two transitions - first if some contact is touching down the system undergoes an impulsive transition, and then if some contact force is trending negative it undergoes a smooth liftoff, but no further transitions are possible at that contact mode and state.

Finally, the last consistency property considers the dynamics of the discrete modes. A general $C^{r}$ hybrid system whose domains are intersecting subsets of some ambient domain (as is true for the self-manipulation hybrid system) need not define a unique execution for a given state from any appropriate mode, even if the hybrid system is deterministic and non-blocking. A given state $\mathbf{T q} \in \mathcal{Q}$ in general is a member of more than one domain, such as the corner point in purple from Figure 6 or, for an arbitrary point, any subset of the current constraint mode. As such, we must ensure that the evolution of the system is not biased by ascribing the "incorrect" mode to that state, nor capable of supporting more than one word (discrete mode sequence) over a given sequence of continuous time trajectories associated with an execution.

Theorem 9. From an initial state $\mathbf{T q}$ at time $t_{0}$ and any contact mode I consistent with that state, i.e. $I \in \mathcal{J}_{\mathbf{q}}:=\{I \in$ $\left.\mathcal{J}: \mathbf{T q} \in D_{I}\right\}$, the execution is uniquely defined (in both state and contact mode) for all $t>t_{0}$ after undergoing up to one hybrid transition.

Proof. The proof considers in turn the two mutually exclusive cases defined by the truth or falsity of the predicate $\operatorname{NTD}(\mathbf{T q}),(22)$, and in each case the execution is uniquely defined due to the uniqueness of the corresponding complementarity problem.

If $\operatorname{NTD}(\mathbf{T q})$ is true then there is some additional constraint $j$ that is impacting, i.e. $\operatorname{TD}(j, \mathbf{T q}),(21)$, where note that $\forall I \in \mathcal{J}_{\mathbf{q}}, j \notin I$. Therefore from any consistent mode $I \in \mathcal{J}_{\mathbf{q}}$, Tq is in some guard $G_{I, J}$, (76), determined by PIV complementarity, $J=\mathrm{CP}_{\mathrm{PIV}}(\mathbf{T q})$, (58). Since the reset map depends on $J$ but not $I$, and $J$ is unique by the impulse-velocity complementarity assumption (A10), the execution continues from the unique point $\left(J, R_{I, J}(\mathbf{T q})\right)$.

If $\operatorname{NTD}(\mathbf{T q})$ is false, then for any $I \in \mathcal{J}_{\mathbf{q}}$ the system could be in a liftoff guard. Consider the mode $J=\mathrm{CP}_{\mathrm{FA}}(\mathbf{T q})$, (38), uniquely defined for a given $\mathbf{T q}$ by the force-acceleration complementarity assumption (A9). If $I=J$ then the state is not in a guard and therefore no reset map is applied. Otherwise $\mathbf{T q} \in G_{I, J}$, (77), and the system undergoes a hybrid transition, though recall that liftoff reset maps are the identity map. In either case the execution continues from the unique point $(J, \mathbf{T q})$.

\subsection{Zeno}

An execution for a hybrid system is referred to as Zeno if it undergoes an infinite number of discrete transitions in finite time (Lygeros et al. 2003, Def. II.3).

Definition 6. An execution $\chi: T \rightarrow \mathcal{D}$ for a hybrid dynamical system $\mathcal{H}=(\mathcal{J}, \Gamma, \mathcal{D}, F, \mathcal{G}, R)$ over a hybrid time trajectory $T=\bigsqcup_{i=1}^{N} T_{i}$ is Zeno if $N=\infty$ and $\sum_{i=1}^{\infty}\left|T_{i}\right|<\infty$.

Zeno executions need not accumulate in a general hybrid system, that is, the $\operatorname{limit}_{\lim } \rightarrow \sup \mathcal{T} \chi(t)$ may be undefined (Zhang et al. 2001, Def. 6). However, Lagrangian systems subject to unilateral constraints give rise to unique 
trajectories defined for all time (Ballard 2000, Thm. 10). We show in Section 3.5.1 that the self-manipulation hybrid dynamical system (Def. 5) models this property through the mechanism of Zeno executions accumulating on a unique limit in the ambient space $T \mathcal{Q}$, from which the hybrid execution proceeds through the next smooth component (and so forward, continuously, through ambient time). Then in Section 4.5 we discuss extensions and connections with results in the literature.

\subsubsection{Accumulation of Zeno executions}

In the following Theorem, we rely on several results originally obtained using sophisticated measure-theoretic techniques (Ballard 2000). At the expense of additional notational overhead, we reproduce the necessary arguments in our hybrid system framework using elementary mathematical machinery.

Theorem 10. Given a self-manipulation hybrid system with a complete connected configuration manifold $\mathcal{Q}$, if the inertia tensor $\overline{\mathbf{M}}$ is non-degenerate and the forces abide by the bound in (19), then the projection of any Zeno execution $\chi: \mathcal{T} \rightarrow \mathcal{D}$ into the ambient state space $T \mathcal{Q}, \pi \circ \chi: \mathcal{T} \rightarrow T \mathcal{Q}$, accumulates on a unique limit,

$$
(\overline{\mathbf{q}}, \dot{\overline{\mathbf{q}}}):=\lim _{t \rightarrow \sup \mathcal{T}} \pi \circ \chi(t) .
$$

Proof. Let $\chi: \mathcal{T} \rightarrow \mathcal{D}$ be a Zeno execution over the hybrid time trajectory $\mathcal{T}=\bigsqcup_{i=1}^{\infty} T_{i}$. With $T_{i} \cap T_{i+1}=\left\{t_{i}\right\}$ for all $i \in \mathbb{N}$, let $\bar{t}=\sup \mathcal{T}<\infty$. For notational convenience in this proof we let $T_{i}=\left[t_{i-1}^{+}, t_{i}^{-}\right]$for all $i \in \mathbb{N}$. This notation is somewhat redundant since $t_{i}^{+}=t_{i}^{-} \in \mathbb{R}$ for all $i \in \mathbb{N}$; we use it to signify that $t_{i}^{-} \in T_{i}$ and $t_{i}^{+} \in T_{i+1}$. Note that $\lim _{i \rightarrow \infty}\left(t_{i}^{-}-t_{i-1}^{+}\right)=0$ since $\lim _{i \rightarrow \infty} t_{i}=\bar{t}$. When there should be no confusion as to the index of the time domain, we abuse notation by suppressing the index and simply write $\dot{\mathbf{q}}(t)$ instead of $\dot{\mathbf{q}}(i, t)$ for $t \in\left[t_{i-1}^{+}, t_{i}^{-}\right]$.

Let $\pi: \mathcal{D} \rightarrow T \mathcal{Q}$ be the canonical projection that simply removes the label from the disjoint union $\mathcal{D}=\amalg_{I \in \mathcal{J}} D_{I}$, and let $(\mathbf{q}, \dot{\mathbf{q}}):=\pi \circ \chi$ denote the velocity and position components of the execution $\chi$. Note that since the reset map, (80), does not change the position, $\mathbf{q}$, the position trajectory $\mathbf{q}: \mathcal{T} \rightarrow \mathcal{Q}$ satisfies,

$$
\left.\mathbf{q}\right|_{T_{i}}\left(t_{i}\right)=\mathbf{q}\left(t_{i}^{-}\right)=\mathbf{q}\left(t_{i}^{+}\right)=\left.\mathbf{q}\right|_{T_{i+1}}\left(t_{i}\right),
$$

for all $i \in \mathbb{N}$, i.e. positions evolve continuously with respect to time. Therefore $\mathbf{q}$ uniquely determines a continuous curve $\widetilde{\mathbf{q}}: T \rightarrow \mathcal{Q}$ over the half-open interval $T=\bigcup_{i=1}^{\infty} T_{i}=[0, \bar{t})$. The restriction $\left.\dot{\mathbf{q}}\right|_{T_{i}}$ is continuous for every $i \in \mathbb{N}$, therefore it uniquely determines a right-continuous curve $\left(\widetilde{\mathbf{q}}, \dot{\mathbf{q}}^{+}\right): T \rightarrow T \mathcal{Q}$. The bound in (19) ensures that the velocity is bounded on finite time horizons,

$$
\bar{v}:=\sup _{t \in T}\left\{\left|\dot{\mathbf{q}}^{+}(t)\right| \overline{\mathbf{M}}\right\}<\infty,
$$

as shown using a sequence of standard results in Appendix E.1 (and adapted from the proof of (Ballard 2000, Thm. 10)). For any nondecreasing Cauchy sequence, $\left\{s_{i}\right\}_{i=1}^{\infty} \subset T$ such that $s_{i} \rightarrow \bar{t}$, the sequence $\left\{\widetilde{\mathbf{q}}\left(s_{i}\right)\right\}_{i=1}^{\infty}$ is also Cauchy since,

$$
\forall n, m \in \mathbb{N}: d_{\overline{\mathbf{M}}}\left(\widetilde{\mathbf{q}}\left(s_{n}\right), \widetilde{\mathbf{q}}\left(s_{m}\right)\right) \leq \int_{s_{m}}^{s_{n}}\left|\dot{\mathbf{q}}^{+}(s)\right|{ }_{\overline{\mathbf{M}}} d s \leq \bar{v}\left|s_{n}-s_{m}\right| .
$$

Therefore the position tends to a unique limit in the ambient state space, i.e. the following limit exists:

$$
\overline{\mathbf{q}}:=\lim _{t \rightarrow \bar{t}} \widetilde{\mathbf{q}}(t) .
$$

Under the simple constraints assumption (A2), the Rank Theorem (Lee 2012, Thm. 4.12) ensures there exists a coordinate chart $(V, \psi)$ near $\overline{\mathbf{q}}$ where $\mathbf{A}_{\mathcal{K}}=[\mathbf{I d}, 0]$. Continuity of $\widetilde{\mathbf{q}}$ ensures there exists $\underline{t} \in T$ for which $\widetilde{\mathbf{q}}([\underline{t}, \bar{t})) \subset V$.

Let $\sigma(\chi)=\left\{J_{i}\right\}_{i=1}^{\infty} \subset \mathcal{J}$ denote the sequence of discrete modes visited by $\chi$ and let $\underline{m}:=\min \left\{i \in \mathbb{N}: t_{i} \geq \underline{t}\right\}$. Specializing the definition of execution of a hybrid system to the self-manipulation system and performing integrationby-parts as in Appendix E.2 we conclude that in coordinates,

$$
\begin{aligned}
\forall i>\underline{m}, t \in\left[t_{i-1}^{+}, t_{i}^{-}\right]: & \overline{\mathbf{M}}(\mathbf{q}(t)) \dot{\mathbf{q}}(t)-\overline{\mathbf{M}}\left(\mathbf{q}\left(t_{i-1}^{+}\right)\right) \dot{\mathbf{q}}\left(t_{i-1}^{+}\right) \\
& =\int_{t_{i-1}}^{t}\left(\Upsilon(\mathbf{q}, \dot{\mathbf{q}})-\overline{\mathbf{N}}(\mathbf{q}, \dot{\mathbf{q}})-\widetilde{\mathbf{C}}(\mathbf{q}, \dot{\mathbf{q}})-\mathbf{A}_{J_{i}}(\mathbf{q})^{T} \lambda_{J_{i}}(\mathbf{q}, \dot{\mathbf{q}})\right) d s, \\
& \overline{\mathbf{M}}\left(\mathbf{q}\left(t_{i}^{+}\right)\right) \dot{\mathbf{q}}\left(t_{i}^{+}\right)-\overline{\mathbf{M}}\left(\mathbf{q}\left(t_{i}^{-}\right)\right) \dot{\mathbf{q}}\left(t_{i}^{-}\right)=-\mathbf{P}_{J_{i}},
\end{aligned}
$$


where $\mathbf{P}_{J_{i}}$ is defined in (24) and for each $\ell \in\{1, \ldots, \mathrm{q}\}$ the $\ell$-th coordinate of the covector $\widetilde{\mathbf{C}} \in T^{*} \mathcal{Q}$ is given by,

$$
\widetilde{\mathbf{C}}^{\ell}(\mathbf{q}, \dot{\mathbf{q}}):=-\frac{1}{2} \sum_{j, k=1}^{\mathrm{q}} \frac{\partial \overline{\mathbf{M}}_{k j}(\mathbf{q})}{\partial \mathbf{q}^{\ell}} \dot{\mathbf{q}}^{k} \dot{\mathbf{q}}^{j}
$$

Recursively substituting using (90) and (87), for any $t \in\left[t_{\underline{m}}, \bar{t}\right)$ with $\bar{m}:=\max \left\{i \in \mathbb{N}: \underline{t} \leq t_{i} \leq t\right\}$, the velocity component of the execution $(\mathbf{q}, \dot{\mathbf{q}}): \mathcal{T} \rightarrow T \mathcal{Q}$ satisfies,

$$
\begin{aligned}
& \overline{\mathbf{M}}(\mathbf{q}(t)) \dot{\mathbf{q}}(t)-\overline{\mathbf{M}}\left(\mathbf{q}\left(t_{\underline{m}}\right)\right) \dot{\mathbf{q}}\left(t_{\underline{m}}\right) \\
& =\int_{t_{m}}^{t}\left(\Upsilon(\mathbf{q}, \dot{\mathbf{q}})-\overline{\mathbf{N}}(\mathbf{q}, \dot{\mathbf{q}})-\widetilde{\mathbf{C}}(\mathbf{q}, \dot{\mathbf{q}})-\mathbf{A}_{J_{\bar{m}}}(\mathbf{q})^{T} \lambda_{J_{m}}(\mathbf{q}, \dot{\mathbf{q}})\right) d s \\
& \quad+\sum_{i=\underline{m}}^{\bar{m}}\left[\int_{t_{i-1}}^{t_{i}}\left(\Upsilon(\mathbf{q}, \dot{\mathbf{q}})-\overline{\mathbf{N}}(\mathbf{q}, \dot{\mathbf{q}})-\widetilde{\mathbf{C}}(\mathbf{q}, \dot{\mathbf{q}})-\mathbf{A}_{J_{i}}(\mathbf{q})^{T} \lambda_{J_{i}}(\mathbf{q}, \dot{\mathbf{q}})\right) d s-\mathbf{P}_{J_{i}}\right] .
\end{aligned}
$$

Noting that for all time $t \in\left(t_{i-1}^{+}, t_{i}^{-}\right)$on the interior of each time interval $i>\underline{m}$ that $\widetilde{\mathbf{q}}(t)=\mathbf{q}(t)$ and $\dot{\widetilde{\mathbf{q}}}^{+}(t)=\dot{\mathbf{q}}(t)$, we conclude that for all $t \in\left[t_{\underline{m}}, \bar{t}\right)$ the right-continuous velocity $\dot{\mathbf{q}}^{+}: T \rightarrow T \mathcal{Q}$ satisfies,

$$
\begin{aligned}
\overline{\mathbf{M}}(\widetilde{\mathbf{q}}(t)) \dot{\overrightarrow{\mathbf{q}}}^{+}(t)-\overline{\mathbf{M}}\left(\widetilde{\mathbf{q}}\left(t_{\underline{m}}\right)\right) \dot{\mathbf{q}}^{+}\left(t_{\underline{m}}\right) \\
\quad=\int_{t_{\underline{m}}}^{t} \Upsilon\left(\widetilde{\mathbf{q}}, \dot{\overrightarrow{\mathbf{q}}}^{+}\right)-\overline{\mathbf{N}}\left(\widetilde{\mathbf{q}}, \dot{\overrightarrow{\mathbf{q}}}^{+}\right)-\widetilde{\mathbf{C}}\left(\widetilde{\mathbf{q}}, \dot{\overrightarrow{\mathbf{q}}}^{+}\right) d s-\int_{t_{\bar{m}}}^{t} \mathbf{A}_{J_{m}}(\widetilde{\mathbf{q}})^{T} \lambda_{J_{\bar{m}}}\left(\widetilde{\mathbf{q}}, \dot{\mathbf{q}}^{+}\right) d s \\
\quad-\sum_{i=\underline{m}}^{\bar{m}}\left[\int_{t_{i-1}}^{t_{i}} \mathbf{A}_{J_{i}}(\widetilde{\mathbf{q}})^{T} \lambda_{J_{i}}\left(\widetilde{\mathbf{q}}, \dot{\mathbf{q}}^{+}\right) d s+\mathbf{P}_{J_{i}}\right] .
\end{aligned}
$$

This equation, (92), is the transcription of (Ballard 2000, Eqn. 36) into our formalism.

Recall that in coordinates $(V, \psi)$ we have $\mathbf{A}_{\mathcal{K}}=[\mathbf{I d}, 0]$ and that $\mathbf{U}(\mathbf{P}) \geq 0 \Rightarrow \mathbf{P} \leq 0, \mathbf{U}(\lambda) \succeq 0 \Rightarrow \lambda \leq 0$. The complementarity conditions, (37) and (49), thus ensure that each component of $\mathbf{A}_{J}^{T} \bar{\lambda}_{J}$ and $\mathbf{P}_{J}$ are non-positive for each $J \in \mathcal{J}$.

We conclude by rearranging (92) (and suppressing dependence on $\widetilde{\mathbf{q}}$ and $\dot{\tilde{\mathbf{q}}}^{+}$) and invoking the bound from Appendix E.3 that there exists $\alpha, \beta \in \mathbb{R}$ such that for each $j \in\{1, \ldots,|\mathcal{K}|\}$,

$$
\begin{aligned}
0 \leq \sum_{i=\underline{m}}^{\bar{m}} & {\left[\int_{t_{i-1}}^{t_{i}} \lambda_{J_{i}}^{j} d s+\mathbf{P}_{J_{i}}^{j}\right]+\int_{t_{\bar{m}}}^{t} \lambda_{J_{m}}^{j} d s=-\left[\overline{\mathbf{M}}^{j}(t) \dot{\widetilde{\mathbf{q}}}^{+}(t)-\overline{\mathbf{M}}^{j}\left(t_{\underline{m}}\right) \dot{\mathbf{q}}^{+}\left(t_{\underline{m}}\right)\right]+\int_{t_{\underline{m}}}^{t} \Upsilon^{j}-\overline{\mathbf{N}}^{j}-\widetilde{\mathbf{C}}^{j} d s } \\
& \leq \alpha+\beta\left(t-t_{\underline{m}}\right) .
\end{aligned}
$$

Therefore the infinite sum,

$$
\sum_{i=\underline{m}}^{\infty}\left[\int_{t_{i-1}}^{t_{i}} \lambda_{J_{i}}^{j} d s+\mathbf{P}_{J_{i}}^{j}\right],
$$

exists and is finite by the Monotone Sequence Theorem (Folland 2002, Thm. 1.16). Thus each coordinate of each term in (92) tends to a unique limit as $t \rightarrow \bar{t}$, i.e. the following limit exists:

$$
\dot{\overline{\mathbf{q}}}:=\lim _{t \rightarrow \bar{t}} \dot{\tilde{\mathbf{q}}}^{+}(t) .
$$

Let $\mathcal{Z} \subset \mathcal{J}$ denote the set of modes visited infinitely often by $\chi$. Since the sequence $\sigma(\chi)=\left\{J_{n}\right\}_{n=1}^{\infty} \subset \mathcal{J}$ of discrete modes visited by $\chi$ is an infinite sequence of elements taken from a finite set, $\mathcal{Z} \neq \emptyset$.

Although the previous result guarantees the continuous state of the system associated with a Zeno execution has a well defined limit, we must further guarantee that it limits on a consistent mode as well. The following result guarantees that this limiting state is indeed achieved in a well defined mode which is also physically meaningful in the sense of being composed of any and all of the constraints that had been active infinitely often during the Zeno execution. 
Corollary 1. Let $\bar{Z}=\bigcup \mathcal{Z}$ denote the set of all constraints visited during a Zeno exection $\chi: T \rightarrow D$. The set is a valid mode, representing the asymptotic contact mode, and the zeno limit $(\overline{\mathbf{q}}, \dot{\overline{\mathbf{q}}})$ from (86) lies in the domain $D_{\bar{Z}}$, i.e.,

$$
\bar{Z} \in \mathcal{J}, \quad \bar{\chi}:=(\overline{\mathbf{q}}, \dot{\overline{\mathbf{q}}}) \in D_{\bar{Z}} .
$$

Proof. We continue with the notational conventions from the Proof of Theorem 10. For all $Z \in \mathcal{Z}$, let $\mathbf{W}_{Z}=$ $\mathbf{A}_{Z}^{T}\left(\mathbf{A}_{Z} \overline{\mathbf{M}}^{-1} \mathbf{A}_{Z}^{T}\right)^{-1} \mathbf{A}_{Z}$ and note: $\left.\mathbf{W}_{Z}=\mathbf{W}_{Z}^{T} \geq 0 ; \mathbf{W}_{Z} \overline{\mathbf{M}}^{-1} \mathbf{W}_{Z}=\mathbf{W}_{Z} ; \dot{\mathbf{q}}\left(t_{i}^{+}\right)=\mathbf{I d}-\overline{\mathbf{M}}^{-1} \mathbf{W}_{J_{i}}\right) \dot{\mathbf{q}}\left(t_{i}^{-}\right)$for all $i \in \mathbb{N} ;$ and $\exists \mathbf{S}_{Z}$ such that $\left(\mathbf{A}_{Z} \overline{\mathbf{M}}^{-1} \mathbf{A}_{Z}^{T}\right)^{-1}=\mathbf{S}_{Z}^{T} \mathbf{S}_{Z}$. Impacts do not increase energy since for all $i \in \mathbb{N}$ :

$$
\begin{aligned}
\frac{1}{2} \dot{\mathbf{q}}\left(t_{i}^{-}\right)^{T} \overline{\mathbf{M}} \dot{\mathbf{q}}\left(t_{i}^{-}\right)-\dot{\mathbf{q}}\left(t_{i}^{+}\right)^{T} \overline{\mathbf{M}} \dot{\mathbf{q}}\left(t_{i}^{+}\right) & =\frac{1}{2} \dot{\mathbf{q}}\left(t_{i}^{-}\right)^{T} \overline{\mathbf{M}} \dot{\mathbf{q}}\left(t_{i}^{-}\right)-\frac{1}{2} \dot{\mathbf{q}}\left(t_{i}^{-}\right)^{T}\left(\mathbf{I d}-\mathbf{W}_{J_{i}} \overline{\mathbf{M}}^{-1}\right) \overline{\mathbf{M}}\left(\mathbf{I d}-\overline{\mathbf{M}}^{-1} \mathbf{W}_{J_{i}}\right) \dot{\mathbf{q}}\left(t_{i}^{-}\right) \\
& =\frac{1}{2} \dot{\mathbf{q}}\left(t_{i}^{-}\right)^{T}\left(\overline{\mathbf{M}}-\overline{\mathbf{M}}+\mathbf{W}_{J_{i}}+\mathbf{W}_{J_{i}}-\mathbf{W}_{J_{i}} \overline{\mathbf{M}}^{-1} \mathbf{W}_{J_{i}}\right) \dot{\mathbf{q}}\left(t_{i}^{-}\right) \\
& =\frac{1}{2} \dot{\mathbf{q}}\left(t_{i}^{-}\right)^{T}\left(2 \mathbf{W}_{J_{i}}-\mathbf{W}_{J_{i}}\right) \dot{\mathbf{q}}\left(t_{i}^{-}\right)=\frac{1}{2} \dot{\mathbf{q}}\left(t_{i}^{-}\right)^{T} \mathbf{W}_{J_{i}} \dot{\mathbf{q}}\left(t_{i}^{-}\right) \geq 0 .
\end{aligned}
$$

Equation (88) implies impacts must extract a finite amount of energy,

$$
\sum_{i=1}^{\infty} \dot{\mathbf{q}}\left(t_{i}^{-}\right)^{T} \mathbf{W}_{J_{i}} \dot{\mathbf{q}}\left(t_{i}^{-}\right)<\infty
$$

and hence in particular,

$$
\lim _{i \rightarrow \infty} \dot{\mathbf{q}}\left(t_{i}^{-}\right)^{T} \mathbf{W}_{J_{i}} \dot{\mathbf{q}}\left(t_{i}^{-}\right)=0
$$

Taking (95) together with,

$$
\dot{\mathbf{q}}^{T} \mathbf{W}_{Z} \dot{\mathbf{q}}=\dot{\mathbf{q}}^{T} \mathbf{A}_{Z}^{T}\left(\mathbf{A}_{Z} \overline{\mathbf{M}}^{-1} \mathbf{A}_{Z}^{T}\right)^{-1} \mathbf{A}_{Z} \dot{\mathbf{q}}=\left|\mathbf{S}_{Z} \mathbf{A}_{Z} \dot{\mathbf{q}}\right| \frac{2}{\mathbf{M}}
$$

implies each Zeno constraint is asymptotically satisfied:

$$
\forall z \in \bar{Z}=\bigcup \mathcal{Z}: \lim _{t \rightarrow \bar{t}} \mathbf{A}_{z} \dot{\mathbf{q}}(t)=0 .
$$

For all constraints $j \in \bar{Z}, j \in Z_{j}$ for at least one $Z_{j} \in \mathcal{Z}$ visited infinitely often in $\chi$. By the definition of $D_{Z_{j}}$,

$$
\forall z \in \bar{Z}: \lim _{t \rightarrow \bar{t}} \mathbf{a}_{z}(\mathbf{q}(t))=0 .
$$

and therefore $\bar{\chi} \in \mathbf{a}_{\bar{Z}}^{-1} \neq \varnothing$. Furthermore for all constraints $j \in \bar{Z}$ and all modes $Z_{j} \in \mathcal{Z}$ containing $j$, the definition of mode $Z_{j}$, (71), requires that $\alpha(j) \in Z_{j} \subset \bar{Z}$. Therefore $\bar{Z} \in \mathcal{J}$ by (71). The domain $D_{\bar{Z}}$ has three requirements, (74), two of which we have already shown to be met by $\bar{\chi}$ in (96) \& (97). Finally, as in (97), for all constraints $k \in \mathcal{K}_{n}$, and all modes $Z \in \mathcal{Z}$, by definition of $D_{Z}$,

$$
\forall k \in \mathcal{K}_{n}: \lim _{t \rightarrow \bar{t}} \mathbf{a}_{k}(\mathbf{q}(t)) \geq 0
$$

Thus $\bar{\chi}=(\overline{\mathbf{q}}, \dot{\overline{\mathbf{q}}}) \in D_{\bar{Z}}$.

\subsubsection{Effect of pseudo-impulse on Zeno executions}

As suggested in Section 2.7, the inclusion of the pseudo-impulse prevents an infinite number of liftoff transitions in a finite amount of time from constraints impinged upon by external forces.

Theorem 11. Let $\chi: \mathcal{T} \rightarrow \mathcal{D}$ be a Zeno execution of a self-manipulation hybrid dynamical system with exactly two contact constraints, so that the limiting set $\bar{Z}=\mathcal{J}$. Under the hypotheses and notation of Theorems 10 \& Corollary 1 , when the pseudo-impulse parameter is positive, $\delta_{t}>0$, we conclude that,

$$
\forall z \in \bar{Z}: \mathbf{U}_{z}\left(\mathbf{A}_{\bar{Z}}^{\dagger}(\overline{\mathbf{q}})(\Upsilon(\overline{\mathbf{q}}, \dot{\overline{\mathbf{q}}})-\overline{\mathbf{C}}(\overline{\mathbf{q}}, \dot{\overline{\mathbf{q}}})-\overline{\mathbf{N}}(\overline{\mathbf{q}}, \dot{\overline{\mathbf{q}}}))\right) \leq 0,
$$

that is, the constraint forces cannot be positive for either constraint at the Zeno limit point.

Proof. We know $\lim _{t \rightarrow \bar{t}} \mathbf{A}_{z} \dot{\mathbf{q}}(t)=0$ for all $z \in \bar{Z}$ by (96). When the liftoff velocity drops below the threshold given implicitly by Theorem 3 , the pseudo-impulse prevents liftoff from constraint $i \in \bar{Z}$ if it violates (99) (i.e., meets the condition (66)). Therefore, the contact force must be negative for both constraints $z \in \bar{Z}$ that undergoes an infinite number of liftoff transitions. 


\section{Discussion}

In this section we discuss the limitations in physical scope incurred by the twelve assumptions of Section 2, implementation details for numerical simulation, and some consequences of the results of this paper through additional examples. Specifically, Section 4.1 reviews on a conceptual level the meaning and implications of the original assumptions. Then Sections $4.2 \& 4.3$ use a number of specific physical examples to elucidate the nature and origin of the more conservative restrictions that are helpful but, we speculate, not necessary to achieve the results of interest in Section 3. Section 4.4 briefly discusses the issues involved with numerically simulating executions of a $C^{r}$ hybrid dynamical system. Finally, Section 4.5 explores the relationship of these assumptions to the Zeno results of Section 3.5.

\subsection{The Base Assumptions}

Most of the assumptions listed in Section 2 are quite common in the modeling of physical systems, especially for models focused on robotics. These limit the scope of the physical settings of interest, and while there are certainly examples of robots that would be poorly modeled by each (many of which are explored in Section 1.3), we believe that there remains a large class of systems that are covered by most if not all of these. Specifically, this class at its core consists of rigid bodies (Assumption A1) under Lagrangian dynamics (A7) with analytic (A3), independent (A2) constraints. That these constraints persist (A4) and are added through plastic impact (A8) are certainly domain specific assumptions, but many robotic tasks involve touching an object or the environment with the goal of continuing that contact in order to do some work.

With these assumptions in place, solving a complementarity problem (A9 \& A10) is the most direct and mathematically tractable way to formulate the change in contact conditions and is in step with a large literature (reviewed in Section 1.3.3). Our insistence on unique solutions to the these problems, key to the consistency conclusions of Section 3.4, has poorly understood consequences except for the case of independent plastic frictionless contacts for which these assumptions are known to hold. The unique structure of the complementarity problems used here allows for the inclusion of other assumptions (in particular massless limbs and the pseudo-impulse, A6 and A11), and Theorems 1 \& 2 (along with Lemma 9) ensures that this form of the complementarity assumptions agrees with the more common versions.

Assumption A7 is imposed both in the interest of the physical scope (Lagrangian dynamics) and mathematical consistency. It arises from the same motivation as the familiar conditions that preclude finite escape in classical dynamical systems but must nevertheless be couched in more technically involved language because of the hybrid setting. Thus we have found it expedient to provide further analysis of what is left behind: the results of Lemmas 6 and 7 guarantee the admissibility of most physically interesting problem instances, but bar (for reasons reflecting the need for a more technical framework, we suspect, rather than mathematical necessity) only the case of nonholonomically constrained massless links.

The remaining assumptions are not imposed to facilitate the definitions and consistency proofs underlying the formal hybrid system (Section 3), but, rather, relate to the practicality of the physical models they can support. We have found the following simplifying (and, strictly speaking, physical fidelity diminishing) assumptions critical to not merely the mathematical tractability but also the qualitative accuracy of the models we use in the robotic settings of interest (as exemplified by the illustrative cases explored below). Thus, the formal results of this paper have been adapted wherever possible to allow for their consistent inclusion. In particular the massless leg assumptions, A5 and A6, are sometimes made for mathematical tractability, but often are not analyzed carefully. Briefly, A5 is tantamount to the assertion that the Lagrange D'Alembert formulation of constrained mechanics should admit smooth generalized coordinates relative to which the kinetic energy is nonsingular, while Assumption A6 similarly requires that any massless degrees of freedom not in contact be assigned some reasonable dynamics. Lemmas 4, 5, \& 8 and Theorems $1 \& 2$ all concern the inclusion of massless limbs with the other assumptions, and the theorems of Section 3.4 ensure that the resulting system is consistent.

Friction in various forms is a common modeling assumption, however the specific setup in Assumption A12 (which divides contacts into either completely slipping or completely sticking but precludes sliding-to-sticking transitions) consists of common components but in a very restricted manner. This particular combination is evidently not the best model of friction for many systems. The existence and uniqueness of solutions to the corresponding complementarity problems for this setup has never been demonstrated and therefore is simply assumed in this paper. 

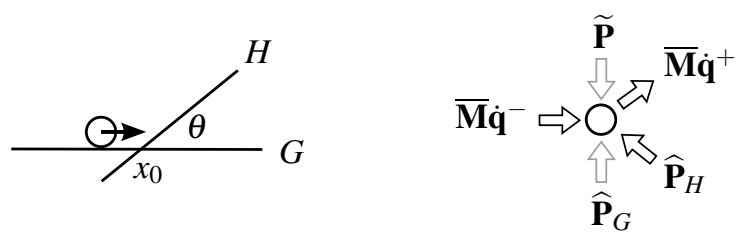

Figure 7: Left: A point sliding along ground $G$ approaches hill $H$. Right: Free body diagram showing impulses at point of contact. Without $\widetilde{\mathbf{P}}$ no positive impulse from the ground $\widehat{\mathbf{P}}_{G}$ is possible for any initial momentum $\overline{\mathbf{M}} \dot{\mathbf{q}}^{-}$and any hill slope $\theta<90^{\circ}$.
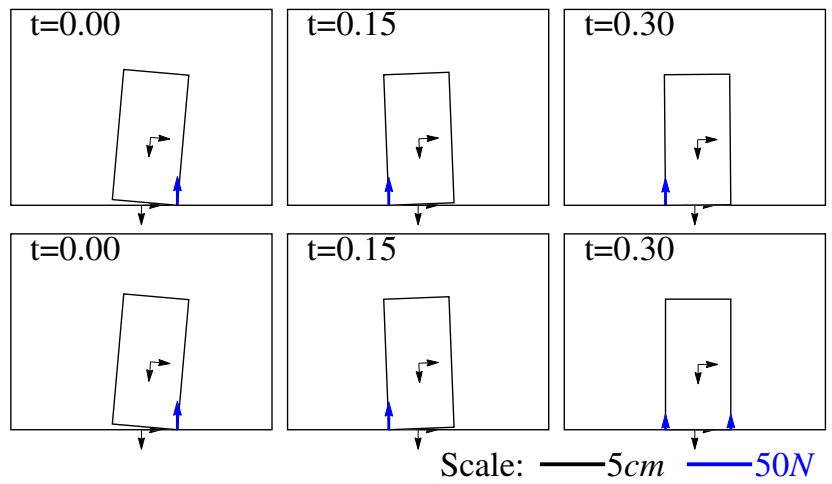

Figure 8: A rocking block (height $h=10 \mathrm{~cm}$, width $w=5 \mathrm{~cm}$, mass $m=5 \mathrm{~kg}$ ) settling on the ground. Top Row: Without pseudo-impulse $\left(\delta_{t}=0\right)$. Bottom Row: With pseudo-impulse $\left(\delta_{t}=0.03\right)$. The execution is identical until the last frame.

Finally, the practical and theoretical implications of the new pseudo-impulse model assumed in A11 have a complicated interplay to whose exploration we devote Section 4.3.

\subsection{Massless Limbs}

One common set of circumstances that satisfy the requirements of the massless limbs assumption (A5) arises when only the robot's most distal link (the finger, lower leg, foot, or in the case of RHex, the entire leg) is massless and the motion of its most distal point is completely constrained when it is on the ground. Although the rank requirement is not limited to this setting, it represents the immediate motivation for our inquiry.

Though there are no truly massless limbs, computing the dynamics using (12)-(13) is numerically more stable than inverting $\overline{\mathbf{M}}_{\varepsilon}$ in the presence of large disparities in limb segment masses (Holmes et al. 2006, Sec. 4.3). This is evidenced by an order of magnitude improvement in the condition number (ratio of largest to smallest singular values) for the RHex model used here (Johnson 2014, Sec. 5.1.1).

\subsection{Pseudo-Impulse}

As a simple example that motivates the need for the pseudo-impulse (Assumption A11), consider a point sliding on the ground as in Figure 7, which hits a hill at some slope $\theta$. The contact impulse from the hill $\widehat{\mathbf{P}}_{H}$ causes the particle to break contact with the ground and leave with some velocity sliding up the hill. This is true for any initial velocity, no matter how small (Lemma 10), and any $\theta<90^{\circ}$. With a pseudo-impulse $\widetilde{\mathbf{P}}$ acting in the direction of gravity, (56), Theorem 3 states that there are initial conditions that result in the point coming to rest with impulses from both the ground and the hill (i.e., all impulses are positive and sum to zero in Figure 7). Note that in this case all quantities scale linearly with mass and as such the solution is the same for any mass. 


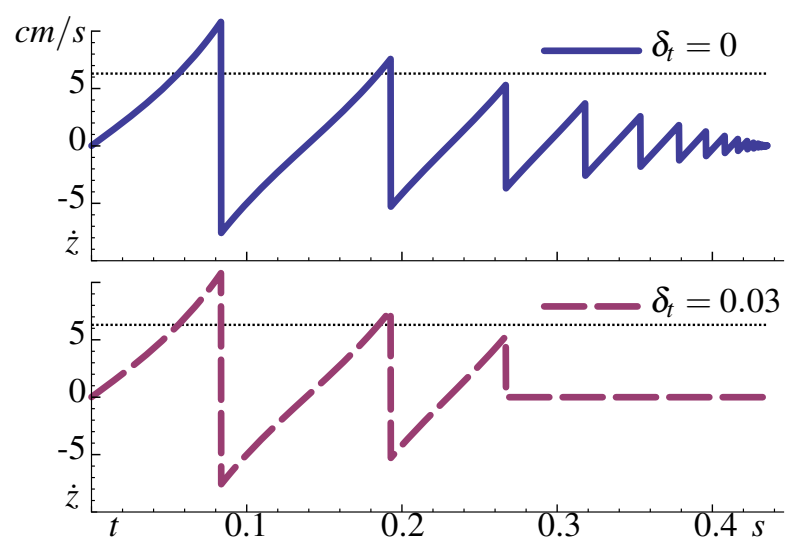

Figure 9: Comparison of the vertical velocity of a settling block for evaluations with and without the pseudo-impulse. The execution is identical until the impact at $t=0.27 \mathrm{~s}$. The pseudo-impulse implicitly bounds the vertical velocity such that an impact at speeds lower than $6.3 \mathrm{~cm} / \mathrm{s}$ causes the block to come to rest, as indicated by the dotted line.

To see how the pseudo-impulse resolves Zeno executions, consider the "rocking block" example of a rectangular rigid body in Figure 8 of width $w$, height $h$, mass $m_{b}$, and inertia $I_{b}$ (where if a uniform distribution is assumed $I_{b}=m_{b}\left(w^{2}+h^{2}\right) / 12$ ), as studied in e.g. Housner (1963), Lygeros et al. (2003), McGeer and Palmer (1989), Yilmaz et al. (2009). As it is falling onto the ground if a corner (labeled "l") is touching down ${ }^{21}$ then the normal direction impulse at that corner when the other corner (labeled "r") hits the ground is,

$$
\mathbf{U}_{l}(\widehat{\mathbf{P}})=\frac{\dot{z}\left(2 I_{b}+m_{b}\left(w^{2}-h^{2}\right) / 2\right)}{w^{2}},
$$

(note that by convention a positive velocity $\dot{z}$ is one that is towards the ground) and the required impulse is negative if,

$$
h^{2}>w^{2}+\frac{4 I_{b}}{m_{b}} \Rightarrow \mathbf{U}_{l}(\widehat{\mathbf{P}})<0,
$$

in which case the contact is broken no matter how slow the block is moving - this is exactly what Lemma 10 predicts. The system exhibits Zeno behavior requiring infinite transitions in finite time as each impact removes some energy but does not immobilize the block, as shown in the upper row of Figure 9 which plots the vertical velocity as the system undergoes a Zeno execution.

Instead if the pseudo-impulse is considered,

$$
\mathbf{U}_{l}(\widehat{\mathbf{P}}+\widetilde{\mathbf{P}})=\frac{\dot{z}\left(2 I_{b}+m_{b}\left(w^{2}-h^{2}\right) / 2\right)}{w^{2}}+\frac{\delta_{t} m_{b} g}{2}
$$

the contact is broken if,

$$
h^{2}>w^{2}+\frac{4 I_{b}}{m_{b}}+\frac{\delta_{t} g w^{2}}{\dot{z}} \Rightarrow \mathbf{U}_{l}(\widehat{\mathbf{P}}+\widetilde{\mathbf{P}})<0,
$$

where as the speed goes to zero $(\dot{z} \rightarrow 0)$ the threshold on height that allows the contact to persist grows and eventually is met - this is exactly the case considered in Theorem 3. This truncation of the Zeno execution shown in the lower row of Figure 9, where for the dimensions used the block comes to rest if the vertical speed at impact is less than $6.3 \mathrm{~cm} / \mathrm{s}$.

Finally, note that the (somewhat restrictive) result in Theorem 11 applies exactly to this rocking block example. The word associated with the Zeno execution of interest alternates between the left and right constraints being active, i.e.,

$$
\sigma(\chi)=\{\{l\},\{r\},\{l\},\{r\}, \ldots\} .
$$

\footnotetext{
${ }^{21}$ In this example the contact points are assumed to resist sliding friction, although when they are both in contact with the ground one of the redundant tangential constraints is dropped. The phenomenon of interest occurs equally well with frictionless contact however the analysis is simpler in the frictional case as presented here.
} 
Since the (gravitational) force violates (99), any value of the pseudo-impulse parameter $\delta_{t}>0$ prevents an infinite number of liftoff (and hence touchdown) transitions for either constraint. We conclude in this case that inclusion of the pseudo-impulse has the effect of truncating the Zeno execution, i.e. preventing an infinite number of discrete transitions in finite time, as explored further in Section 4.5.

\subsection{Numerical Simulation}

As noted in the Introduction, this paper is focused on constructing from the rigid body dynamics assumptions of Section 2 a hybrid dynamical systems model in Section 3 that has formally established, useful mathematical properties. However, as is generally true of classical ODE models in engineering applications, it often lends additional insight to approximate an execution of this hybrid system through numerical simulation. For example, simulations of this model are used to generate Figures 1, 3, 4, 5, 8, 9, and 10. A full exploration of the issues involved in numerical simulation lies far outside the scope of the present paper. In this section we briefly describe some of the most critical details needed to simulate executions of our self-manipulation hybrid dynamical system.

The simulations presented in this paper were implemented in Mathematica ${ }^{22}$ using a conventional event-driven scheme $^{23}$. An execution of the self-manipulation hybrid dynamical system, Definition 4, is calculated for each interval of the hybrid time domain, Definition 3, sequentially. The flow in that particular contact mode, $F_{I},(75)$, is integrated from the state at the initial time as an ordinary or differential-algebraic equation Ascher and Petzold (1998), as appropriate, using the NDSolve command with default parameters. The integration is stopped when the state reaches any guard, $G_{I},(78)$, as detected by the WhenEvent command. Separate events are used to test the new touchdown predicate, NTD, (22), and liftoff predicate, LO, (81), which make up the guard (described in further detail below). When an event is detected the integration stops and the subsequent contact mode is determined by evaluating the appropriate complementarity problem according to (79). Using this combined guard set requires fewer conditions than testing for each guard separately, however the resulting executions are identical. Finally the reset map, $R_{I, J},(80)$, is applied and the numerical integration continues again in the next time interval in contact mode $J$.

The numerical implementation of the two event predicate detections can be simplified from the full definition used in the above proofs. Specifically, the touchdown predicate may be checked with an event $\mathbf{a}_{k}(\mathbf{q})<0$, which given the domain constraints, (74), becomes true at the moment the conditions of (21) hold. Similarly, by Lemma 3, the liftoff predicate (which includes a trending condition, $\prec$ ) may be checked with an event $\mathbf{U}_{k}\left(\lambda_{I}\right)<0$, which becomes true at the moment the conditions of (81) hold. Numerically determining these event times and states involves integrating beyond the event (since the relevant quantities can be formally extended outside the domain $\mathcal{D}$ ) and then stepping back to approximate the zero crossing to some desired precision using a numerical root-finding algorithm ${ }^{24}$.

Once an event is detected, the complementarity problem, either $\mathrm{CP}_{\mathrm{PIV}}$ or $\mathrm{CP}_{\mathrm{FA}}$, can be solved in many ways, see e.g. Cottle and Dantzig (1968), Pang et al. (1996). For small systems of contacts, a simple but inefficient method is to simply check the truth value of the predicate, either PIV, (58), or FA, (38), for all possible subsequent modes, $J \subseteq \mathcal{I}$, and choose the unique mode where the predicate is true.

The scope, $\mathcal{I}$, (33), of these complementarity problems can also be simplified, as noted in Section 2.6. For liftoff guards, in general it suffices to check simply the active constraints, $I \subseteq \mathcal{I}$. Any constraints that are not in $I$ and therefore not algebraically guaranteed to satisfy the equality condition in (33) will, due to numerical error, only be close. Using $I$ instead of $\mathcal{I}$ will miss cases such as Figure 2 (d), wherein a constraint $j$ that is not in the current active mode $(j \notin I)$ satisfies the scope in $(33)(j \in \mathcal{I})$. However these examples are not generic as any perturbation in the state or constraint resolves this problem - they make up a set of measure zero which we do not expect can be reached numerically. Similarly the scope for touchdown guards may be taken as the active set plus any impacting constraints, as stated in (48).

\footnotetext{
22 http: //www.wolfram.com/mathematica/

23 Proposed originally in Witsenhausen (1966) and subsequently popularized by Back et al. (1993), Shampine et al. (1991), this simulation algorithm for hybrid systems was proven to converge to orbitally stable trajectories that encounter (i) isolated transitions by Tavernini (1987) and (ii) simultaneous transitions by Burden et al. (2015a). We refer the reader to Burden et al. (2015a) as a comprehensive reference for the definition of orbitally stable trajectories, implementation details for the simulation algorithm, and proofs of convergence of simulations to executions.

24 Although there exist pathological cases wherein this scheme determines event times inaccurately (or fail to detect events entirely), so long as the desired execution satisfies a mild orbital stability property this scheme is guaranteed to succeed (Burden et al. 2015a, Theorem 27).
} 


\subsection{Zeno convergence results}

It is instructive to contrast Theorems 10 and 11 and Corollary 1 with the completion results in Or and Ames (2011). For Lagrangian systems subject to plastic impact with a single unilateral constraint, the completion of the simple Lagrangian hybrid system in Or and Ames (2011) coincides with the self-manipulation hybrid system we develop in Section 3.2. Specifically, the completion is a hybrid dynamical system (in the sense of Definition 2 with one constrained and one unconstrained mode. Transition from the unconstrained to the constrained mode occurs at impact; outward-trending forces trigger the transition back to the unconstrained mode. Our self-manipulation system can therefore be viewed as a generalization of the completion to Lagrangian systems undergoing plastic impact with an arbitrary number of unilateral constraints, a situation not considered in Or and Ames (2011). In connection with the numerical simulation literature discussed in Section 1.3.1, we note that (Stewart 1998, Lem. 12) provides an accumulation result for time-stepping algorithms that is analogous to our Corollary 1.

We further clarify the relationship between our contributions and the results in Or and Ames (2011) in the case of purely inelastic (i.e., plastic) impact. Although the (un-completed) simple Lagrangian hybrid system allows plastic impacts (i.e., a coefficient of restitution $e=0$ in (Or and Ames 2011, Eqn. 4)), the definition of the guard in (Or and Ames 2011, Sec. II-A-3) implies that every plastic impact is a Zeno event - every $(\mathbf{q}, \dot{\mathbf{q}}) \in T \mathcal{Q}$ for which $\mathbf{a}(\mathbf{q})=0$ and $\mathbf{A}(\mathbf{q}) \dot{\mathbf{q}}=0$ is a fixed point of the reset map in (Or and Ames 2011, Eqn. 4). This stands in contrast to our guard definition (76)-(77), where we have excised such points from the domains of the reset maps. In plain language, we ensure that constraints may persist after an impact without instantaneously triggering Zeno events. As an illustration, consider just a single impact event in the rocking block of Figure 8. In the simple Lagrangian hybrid system of Or and Ames (2011), plastic impact at time $t \in \mathbb{R}$ results in a Zeno execution over a hybrid time trajectory $\mathcal{T}=\coprod_{i=1}^{\infty}\{t\}$ that spans zero (continuous) time, thus conflicting with Theorem 8 (before, possibly, completion and continuing exection to the Zeno execution considered in Section 4.3). In our self-manipulation hybrid system, the execution continues past this impact as illustrated in Figure 9 (top) by transitioning to a constrained mode. We note that the behavior of our system (and, equivalently, the completion from Or and Ames (2011)) is consistent with the analysis of the rocking block in (Housner 1963, Sec. 2).

We also comment on the relationship between the truncation effect introduced by our pseudo-impulse and the reliable truncation proposed in (Or and Ames 2011, Def. 6). The pseudo-impulse we proposed in Section 2.7 prevents an infinite number of isolated liftoffs in finite time from pairs of constraints impinged upon by the external forces; this is the content of Theorem 11. In (Or and Ames 2011, Def. 6), reliable truncation conditions were shown to yield simulated executions that approximate a Zeno execution to specified precision; this is the content of (Or and Ames 2011, Thm. 3). Thus our contribution is a phenomenological heuristic that augments the hybrid system to prevent some Zeno executions from arising (specifically, those Zeno executions that only involve two constraints impinged upon by external forces). The contribution in (Or and Ames 2011, Sec. V) is a formal guarantee of simulation accuracy for Zeno executions in the original hybrid system. We have yet to determine the "reliability" of our truncation in this sense (though, as noted in the discussion following Theorem 11, the psuedo-impulse truncation is reliable in this sense for the rocking block).

It is possible to relax the hypotheses in Theorem 10 in several ways that ensure the results in Section 3.5.1 still hold. It is straightforward to allow time-dependent forcing (as in Ballard 2000, Thm. 10) so long as the applied and potential forces obey the estimate,

$$
\forall(\mathbf{q}, \dot{\mathbf{q}}) \in T \mathcal{Q}:|\Upsilon(t, \mathbf{q}, \dot{\mathbf{q}})-\overline{\mathbf{N}}(t, \mathbf{q}, \dot{\mathbf{q}})|_{\overline{\mathbf{M}}^{-1}} \leq \ell(t)\left(1+|\dot{\mathbf{q}}|_{\overline{\mathbf{M}}}+d_{\overline{\mathbf{M}}}\left(\mathbf{q}_{0}, \mathbf{q}\right)\right)
$$

where $\ell: \mathbb{R} \rightarrow \mathbb{R}$ is nonnegative and locally integrable. Fully-actuated massless limbs can be included by constraining their motion with respect to the body degrees-of-freedom, e.g. through the use of "mirror laws" (Buehler et al. 1994), so long as the forces required to enforce the desired motion obeys the estimate in (4.5). Care must be taken to allow the forcing to depend on the contact mode, since it is possible to introduce "sliding modes" wherein limbs cycle infinitely often between constrained and unconstrained modes at a single time instant; we discuss this issue further in Section 4.6.

Finally, as we have not yet been able to construct an example wherein a constraint that meets (99) is involved in a Zeno execution, we speculate that the pseudo-impulse truncates a larger class of Zeno executions than handled by Theorem 11. Such an extension would require a careful treatment of the interaction between the complementarity conditions and the Zeno execution, resulting in either a proof that (99) can not hold for a constraint undergoing Zeno or conditions for Zeno executions involving more than two contact constraints. 

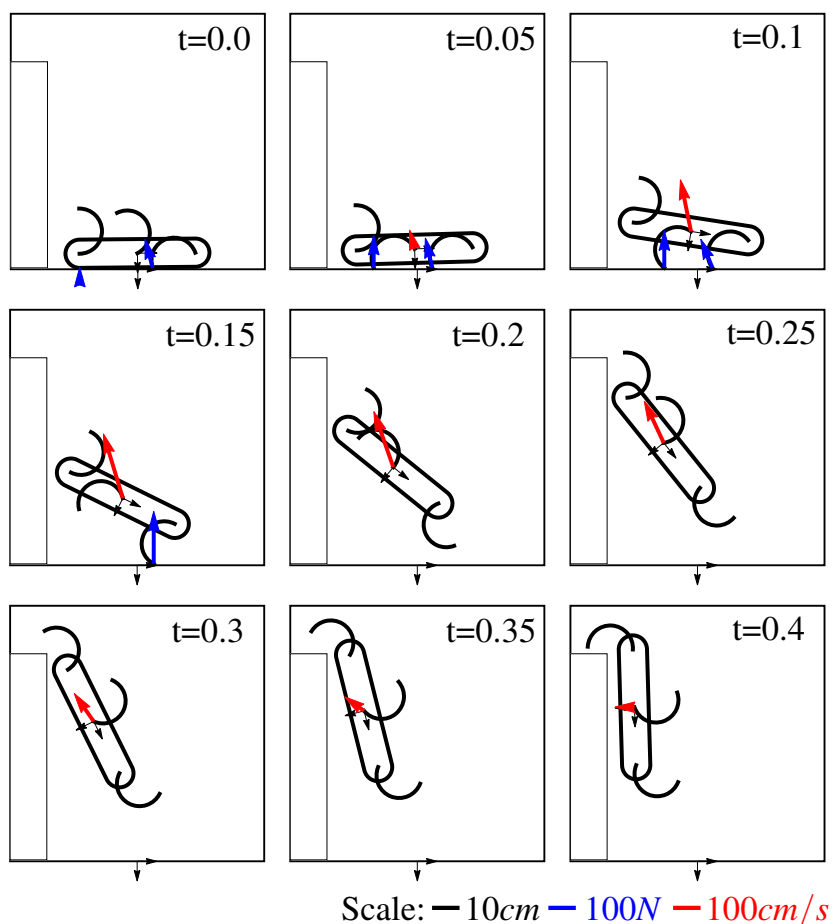

Figure 10: Keyframes from RHex simulation leaping onto a $73 \mathrm{~cm}$ ledge. Blue arrows show contact forces while the red arrow shows body velocity. The coefficient of friction is $\mu=0.8$ and the relative leg timing is $t_{2}=0.06 \mathrm{~s}$.

\subsection{Contact-dependent forcing}

The developments in Sections 2 and 3 allow, in principle, for the applied forces $\Upsilon_{I}$ to depend on the set of active constraints $I \in \mathcal{J}$. This is a desirable feature of our formalism since many extant robots sense their contact state with the world and accordingly alter their actuator commands. This also enables the separate handling of massless limbs that make or break contact with the ground, Assumptions A5 and A6. The hybrid system formalism provides a direct route to incorporate this sort of feedback. Indeed, so long as one can ensure that the complementarity assumptions hold, A9 and A10, then the self-manipulation system (Def. 5) has disjoint guards (Thm. 5) and hence is deterministic (Thm. 6) and non-blocking (Thm. 7). Note, however, that the complementarity problems do not depend on contact mode, and so care must be used to enable contact-dependent forcing that does not break these assumptions, otherwise the execution may alternate between two adjacent modes.

\section{Conclusion}

The hybrid system model presented here provides for the consistent inclusion of many common simplifying physical assumptions, including rigid bodies and plastic impacts, as well as some less common assumptions, such as the pseudo-impulse. These assumptions are well understood to be only approximations to the real physics: our central contribution is to develop sufficiently compatible refinements of previously investigated versions as to obviate their erstwhile conflicts. Nevertheless, this refined model is still able to capture qualitatively many behaviors of interest in robotics - not merely the familiar steady state tasks (e.g. Buehler et al. 1994, Holmes et al. 2006) but also transitional maneuvers such as, archetypally, the leap onto a ledge shown in Figure 10 (a behavior first demonstrated in Johnson and Koditschek (2013b)). Simulation results such as these suggest the descriptive power of our refined collection of physical assumptions, while the consistency properties of Section 3.4 ensure that they avoid these potential conflicts. However, as noted in the text, there remain a few cases where the formal proofs included here are limited to a still further constrained subset of mechanical settings than admitted by these assumptions, most notably Theorems 3, 8, $10, \& 11$. As noted at several points throughout the text, we believe that the conclusions remain true under the broader conditions (i.e., those listed in the assumptions themselves), but more general proofs of these properties remain an 
open research question.

Including such explicit assumptions makes it clear that certain extensions of this model satisfying these assumptions are trivially admissible, such as more complicated rolling contact conditions, (Murray et al. 1994, Sec. 5.2.1), over rough (though still semi-analytic) terrain. At the same time extensions that violate these assumptions require that some of the formal proofs be reconsidered, for example elastic impacts. One assumption that is often relaxed is the persistence of contact, Assumption A4, which precludes the use of time-stepping formulations (e.g., presented in Anitescu and Potra 1997, Stewart and Trinkle 1996) that have gained in popularity as a modeling and simulation framework. The remaining assumptions do not explicitly depend on Assumption A4, and so it may be possible to extend some of the results from Section 2 to these settings (although the massless leg conditions, Assumptions A5 \& A6, and the pseudo-impulse assumption, Assumption A11, may prove challenging to maintain).

Indexing the contact mode as a subset of the possible contact constraints suggests a natural simplicial topology (Hatcher 2002) over these contact modes, as first suggested in Johnson and Koditschek (2013b). This organization of the hybrid system should enable the inspection of structural properties of the system as a whole. Furthermore the various guard sets imply a refinement of the domains into disjoint sets that reach a unique next guard (or remain in that mode forever). More broadly, we believe that this physically motivated hybrid system definition, formal consistency now established, invites study as a mathematical object whose properties may likely yield formal insights into the nature of these mechanical systems and promote the design of more complex robot behaviors that can exploit them.

\section{Acknowledgments}

This was supported in part by the ARL/GDRS RCTA project under Cooperative Agreement Number W911NF-10-20016. The authors would like to thank the reviewers and editors for their substantial time and expert consideration of this work, as well as their numerous helpful suggestions for sharpening the claims and improving the readability of the final paper. We also thank Avik De, Andy Ruina, Russ Tedrake, and Michael Posa for helpful related discussions over the past few years. An earlier version of parts of this work previously appeared in Johnson (2014).

\section{Appendix}

\section{A Proof of Lemma 2}

Proof. First consider $\left(h(x) \prec_{F} 0\right) \Rightarrow\left(g(x) h(x) \prec_{F} 0\right)$. Let $m_{h}$ be the index of the first nonzero derivative in the definition of $h(x) \prec_{F} 0$, (3), and so for all $\ell<m_{h}$ the $\ell^{\text {th }}$ Lie derivative is zero, $\left(\mathcal{L}_{F}^{\ell} h\right)(x)=0$. Therefore we also have,

$$
\left(\mathcal{L}_{F}^{\ell}(g \cdot h)\right)(x)=\sum_{k=0}^{\ell}\left(\begin{array}{l}
\ell \\
k
\end{array}\right)\left(\mathcal{L}_{F}^{\ell-k} g(x)\right) \cdot\left(\mathcal{L}_{F}^{k} h(x)\right)=\sum_{k=0}^{\ell}\left(\begin{array}{l}
\ell \\
k
\end{array}\right)\left(\mathcal{L}_{F}^{\ell-k} g(x)\right) \cdot(0)=0,
$$

and similarly,

$$
\left(\mathcal{L}_{F}^{m_{h}} g \cdot h\right)(x)=\sum_{k=0}^{m_{h}}\left(\begin{array}{c}
m_{h} \\
k
\end{array}\right)\left(\mathcal{L}_{F}^{m_{h}-k} g(x)\right) \cdot\left(\mathcal{L}_{F}^{k} h(x)\right)=g(x) \cdot\left(\mathcal{L}_{F}^{m_{h}} h(x)\right)
$$

where since $g(x)>0, g(x) \cdot\left(\mathcal{L}_{F}^{m_{h}} h(x)\right)<0 \Leftrightarrow\left(\mathcal{L}_{F}^{m_{h}} h(x)\right)<0$. Therefore $\left(h(x) \prec_{F} 0\right) \Rightarrow\left(g(x) h(x) \prec_{F} 0\right)$.

Now consider $\left(g(x) h(x) \prec_{F} 0\right) \Rightarrow\left(h(x) \prec_{F} 0\right)$. Let $m_{g h}$ be the index of the first nonzero derivative in the definition of $g(x) \cdot h(x) \prec_{F} 0$, (3). The proof proceeds by strong induction on $\ell$, where $0 \leq \ell \leq m_{g h}$, relative to the proposition,

$$
\left(\mathcal{L}_{F}^{\ell} g \cdot h\right)(x)=g(x) \mathcal{L}_{F}^{\ell} h(x),
$$

i.e. that the $\ell^{\text {th }}$ Lie derivative of the product is equal to the product of positive function, $g(x)$, with the $\ell^{\text {th }}$ Lie derivative of the other factor, $h(x)$. The base case is trivial, as for the $0^{t h}$ derivative,

$$
\left(\mathcal{L}_{F}^{0} g \cdot h\right)(x)=g(x) \mathcal{L}_{F}^{0} h(x)=g(x) h(x) .
$$

If $m_{g h}=0$, then $g(x) \cdot h(x)<0$, but since $g(x)>0$ we get that $h(x)<0$ and therefore $h(x) \prec 0$. If instead $m_{g h}>0$, then $g(x) \cdot h(x)=0$ and therefore $h(x)=0$. For the inductive step, suppose that the statement is true for all $k<\ell$, 
implying that,

$$
\left(\mathcal{L}_{F}^{k} g \cdot h\right)(x)=g(x) \mathcal{L}_{F}^{k} h(x)=0,
$$

(as recall that $\ell \leq m_{g h}$, and therefore, $\mathcal{L}_{F}^{k} h(x)=0$. Thus,

$$
\left(\mathcal{L}_{F}^{\ell}(g \cdot h)\right)(x)=\sum_{k=0}^{\ell}\left(\begin{array}{l}
\ell \\
k
\end{array}\right)\left(\mathcal{L}_{F}^{\ell-k} g(x)\right) \cdot\left(\mathcal{L}_{F}^{k} h(x)\right)=g(x) \cdot\left(\mathcal{L}_{F}^{\ell} h(x)\right)
$$

and the induction complete, we now conclude that the proposition, (107), holds for all $\ell \leq m_{g h}$.

If $g(x) h(x) \prec_{F} 0$, then by (3) for all $\ell<m_{g h},\left(\mathcal{L}_{F}^{\ell}(g \cdot h)(x)\right)=0$, and so using (107) and $g(x)>0$, we conclude that $\mathcal{L}_{F}^{\ell} h(x)=0$. Similarly for $\ell=m_{g h},\left(\mathcal{L}_{F}^{m_{g h}}(g \cdot h)(x)\right)<0 \Leftrightarrow \mathcal{L}_{F}^{m_{g h}} h(x)<0$. Taken together, these are exactly the conditions for $h(x) \prec_{F} 0$, (3), and so $\left(g(x) h(x) \prec_{F} 0\right) \Rightarrow\left(h(x) \prec_{F} 0\right)$.

\section{B Linear Algebra}

For additional notes on the Schur complement and block matrix inverse, see e.g. Cottle (1974), Lu and Shiou (2002), or Jo et al. (2004). Consider a block matrix $M$ defined as,

$$
M:=\left[\begin{array}{cc}
E & F \\
G & H
\end{array}\right] .
$$

If $E$ is nonsingular, then the Schur complement of $E$ in $M$ is,

$$
S_{E}:=H-G E^{-1} F,
$$

which is sometimes written as $(M \mid E)$.

If $M$ is also nonsingular, the inverse of $M$ is,

$$
\left[\begin{array}{cc}
E & F \\
G & H
\end{array}\right]^{-1}=\left[\begin{array}{cc}
E^{-1}+E^{-1} F S_{E}^{-1} G E^{-1} & -E^{-1} F S_{E}^{-1} \\
-S_{E}^{-1} G E^{-1} & S_{E}^{-1}
\end{array}\right] .
$$

In particular when $\overline{\mathbf{M}}$ is invertible the block matrix inverse of (8) can be written as,

$$
\left[\begin{array}{cc}
\overline{\mathbf{M}}_{J}^{\dagger} & \mathbf{A}_{J}^{\dagger T} \\
\mathbf{A}_{J}^{\dagger} & \Lambda_{J}
\end{array}\right]:=\left[\begin{array}{cc}
\overline{\mathbf{M}} & \mathbf{A}_{J}^{T} \\
\mathbf{A}_{J} & \mathbf{0}_{J \times J}
\end{array}\right]^{-1}=\left[\begin{array}{cc}
\overline{\mathbf{M}}^{-1}-\overline{\mathbf{M}}^{-1} \mathbf{A}^{T}\left(\mathbf{A} \overline{\mathbf{M}}^{-1} \mathbf{A}^{T}\right)^{-1} \mathbf{A} \overline{\mathbf{M}}^{-1} & \overline{\mathbf{M}}^{-1} \mathbf{A}^{T}\left(\mathbf{A} \overline{\mathbf{M}}^{-1} \mathbf{A}^{T}\right)^{-1} \\
\left(\mathbf{A} \overline{\mathbf{M}}^{-1} \mathbf{A}^{T}\right)^{-1} \mathbf{A} \overline{\mathbf{M}}^{-1} & -\left(\mathbf{A} \overline{\mathbf{M}}^{-1} \mathbf{A}^{T}\right)^{-1}
\end{array}\right] .
$$

Where when $\overline{\mathbf{M}}$ is positive definite, so is $\left(\mathbf{A} \overline{\mathbf{M}}^{-1} \mathbf{A}^{T}\right)^{-1}$, and therefore $\Lambda$ is negative definite. Similarly, when $\overline{\mathbf{M}}$ is only positive semi-definite, $\Lambda$ is negative semi-definite.

A common refinement to this inverse that comes up when considering some constraint sets $J$ and $K$ such that $K=J \cup\{k\}$ is ${ }^{25}$,

$$
\begin{aligned}
& \mathbf{A}_{K}=\left[\begin{array}{l}
\mathbf{A}_{J} \\
\mathbf{A}_{k}
\end{array}\right],
\end{aligned}
$$

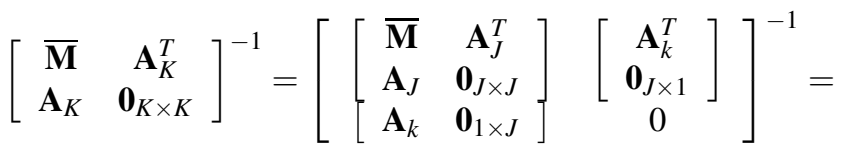

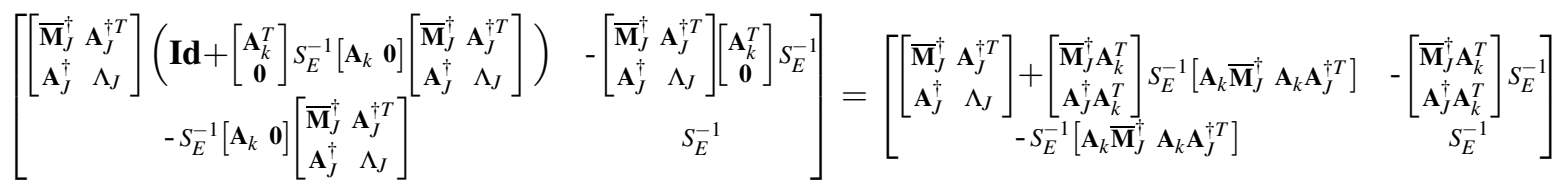

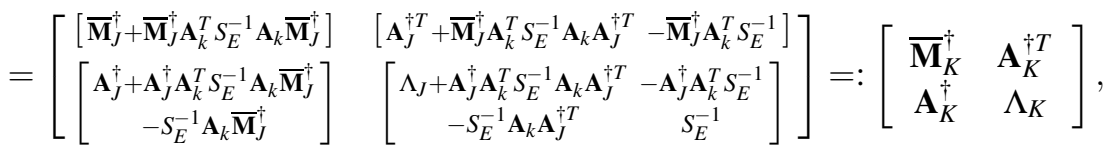

$$
\begin{aligned}
& S_{E}:=0-\left[\begin{array}{ll}
\mathbf{A}_{k} & \mathbf{0}
\end{array}\right]\left[\begin{array}{cc}
\overline{\mathbf{M}}_{J}^{\dagger} & \mathbf{A}_{J}^{\dagger T} \\
\mathbf{A}_{J}^{\dagger} & \Lambda_{J}
\end{array}\right]\left[\begin{array}{c}
\mathbf{A}_{k}^{T} \\
\mathbf{0}
\end{array}\right]=-\mathbf{A}_{k} \overline{\mathbf{M}}_{J}^{\dagger} \mathbf{A}_{k}^{T} .
\end{aligned}
$$

\footnotetext{
${ }^{25}$ Note that the Schur complement, $S_{E}$, used here is with respect to the blocks used in (116) as defined explicitly in (118).
} 
Note that when both the matrix and the first block in (116) are invertible, $S_{E}$ must be non-zero as $S_{E}^{-1}$ is an element of the inverse in (117). Since $\Lambda_{K}$ is negative semi-definite, so are its principle minors, in particular $S_{E}^{-1}$. Therefore $\mathbf{A}_{k} \overline{\mathbf{M}}_{J}^{\dagger} \mathbf{A}_{k}^{T}$, as a positive semi-definite and non-zero scalar, is a positive number. This final expansion, (117), expresses the components of $\mathbf{A}_{K}^{\dagger}, \overline{\mathbf{M}}_{K}^{\dagger}$, and $\Lambda_{K}$ in terms of $\mathbf{A}_{J}^{\dagger}, \overline{\mathbf{M}}_{J}^{\dagger}$, and $\Lambda_{J}$ together with the added constraint $\mathbf{A}_{k}$.

\section{B.1 Proof of Lemma 4}

Proof. Define some set of generalized coordinates (as in (Johnson and Koditschek 2013a, Sec. II.G)), y, such that $\dot{\mathbf{y}}=\mathbf{Y} \dot{\mathbf{q}}$ and that the Jacobian of the corresponding implicit function is defined so that $\dot{\mathbf{q}}=\mathbf{H} \dot{\mathbf{y}}$,

$$
\mathbf{H}=\left[\begin{array}{l}
\mathbf{A} \\
\mathbf{Y}
\end{array}\right]^{-1}\left[\begin{array}{c}
0 \\
\mathbf{I d}
\end{array}\right]
$$

For this proof we need to show that $\tilde{\mathbf{M}}=\mathbf{H}^{T} \overline{\mathbf{M}} \mathbf{H}$ is invertible if and only if $\left[\begin{array}{cc}\overline{\mathbf{M}} & \mathbf{A}^{T} \\ \mathbf{A} & 0\end{array}\right]$ is. The Rank Theorem (Lee 2012, Thm. 4.12) implies there exists a parameterization such that the constraint can be decoupled into a full rank $\mathrm{c} \times \mathrm{c}$ subblock, $\mathbf{A}=\left[\begin{array}{ll}\mathbf{B} & 0_{c \times e}\end{array}\right]$, and therefore we choose a parameterization such that, $\mathbf{Y}=\left[\begin{array}{ll}0_{e \times c} & \mathbf{I d}\end{array}\right]$. Thus,

$$
\begin{gathered}
\mathbf{H}=\left[\begin{array}{cc}
\mathbf{B} & 0_{c \times e} \\
0_{e \times c} & \mathbf{I d}_{e}
\end{array}\right]^{-1}\left[\begin{array}{c}
0 \\
\mathbf{I} \mathbf{d}_{e}
\end{array}\right]=\left[\begin{array}{cc}
\mathbf{B}^{-1} & 0_{c \times e} \\
0_{e \times c} & \mathbf{I d}_{e}
\end{array}\right]\left[\begin{array}{c}
0 \\
\mathbf{I} \mathbf{d}_{e}
\end{array}\right]=\left[\begin{array}{c}
0 \\
\mathbf{I d}_{e}
\end{array}\right], \\
\tilde{\mathbf{M}}=\mathbf{H}^{T} \overline{\mathbf{M}} \mathbf{H}=\left[\begin{array}{ll}
0 & \mathbf{I d}_{e}
\end{array}\right]\left[\begin{array}{ll}
\overline{\mathbf{M}}_{11} & \overline{\mathbf{M}}_{12} \\
\overline{\mathbf{M}}_{21} & \overline{\mathbf{M}}_{22}
\end{array}\right]\left[\begin{array}{c}
0 \\
\mathbf{I d}_{e}
\end{array}\right]=\overline{\mathbf{M}}_{22},
\end{gathered}
$$

and so the requirement is that $\tilde{\mathbf{M}}$ is invertible reduces down to simply requiring that $\overline{\mathbf{M}}_{22}$ is invertible.

On the other hand we have,

$$
\left[\begin{array}{cc}
\overline{\mathbf{M}} & \mathbf{A}^{T} \\
\mathbf{A} & 0
\end{array}\right]=\left[\begin{array}{ccc}
\overline{\mathbf{M}}_{11} & \overline{\mathbf{M}}_{12} & \mathbf{B}^{T} \\
\overline{\mathbf{M}}_{21} & \overline{\mathbf{M}}_{22} & 0 \\
\mathbf{B} & 0 & 0
\end{array}\right]
$$

Since $\mathbf{B}$ is full rank then the invertability of this matrix again reduces to simply invertibility of $\overline{\mathbf{M}}_{22}$ (e.g. Lu and Shiou 2002, Corollary 3.3), and thus the conditions are equivalent.

\section{B.2 Proof of Lemma 5}

Proof. Recall that $\lim _{\varepsilon \rightarrow 0} \overline{\mathbf{M}}_{\varepsilon}=\overline{\mathbf{M}}$ and that $\overline{\mathbf{M}}_{\varepsilon}$ is invertible for all $\varepsilon \in(0, \bar{\varepsilon})$, for some $\bar{\varepsilon}>0$. For all $\varepsilon \geq 0$, define $\overline{\mathbf{M}}_{\varepsilon}^{\dagger}, \mathbf{A}_{\varepsilon}^{\dagger}$, and $\Lambda_{\varepsilon}$ by replacing $\overline{\mathbf{M}}$ with $\overline{\mathbf{M}}_{\varepsilon}$ in (8). Using (14)-(16) we rewrite the dynamics, (12)-(13),

$$
\begin{aligned}
\lambda & =\mathbf{A}^{\dagger}(\Upsilon-\overline{\mathbf{C}} \dot{\mathbf{q}}-\overline{\mathbf{N}})-\Lambda \dot{\mathbf{A}} \dot{\mathbf{q}}=\lim _{\varepsilon \rightarrow 0} \mathbf{A}_{\varepsilon}^{\dagger}(\Upsilon-\overline{\mathbf{C}} \dot{\mathbf{q}}-\overline{\mathbf{N}})-\Lambda_{\varepsilon} \dot{\mathbf{A}} \dot{\mathbf{q}} \\
& =\lim _{\varepsilon \rightarrow 0}\left(\left(\mathbf{A} \overline{\mathbf{M}}_{\varepsilon}^{-1} \mathbf{A}^{T}\right)^{-1} \mathbf{A} \overline{\mathbf{M}}_{\varepsilon}^{-1}\right)(\Upsilon-\overline{\mathbf{C}} \dot{\mathbf{q}}-\overline{\mathbf{N}})+\left(\mathbf{A} \overline{\mathbf{M}}_{\varepsilon}^{-1} \mathbf{A}^{T}\right)^{-1} \dot{\mathbf{A}} \dot{\mathbf{q}} \\
& =\lim _{\varepsilon \rightarrow 0}\left(\mathbf{A} \overline{\mathbf{M}}_{\varepsilon}^{-1} \mathbf{A}^{T}\right)^{-1}\left(\mathbf{A} \overline{\mathbf{M}}_{\varepsilon}^{-1}(\Upsilon-\overline{\mathbf{C}} \dot{\mathbf{q}}-\overline{\mathbf{N}})+\dot{\mathbf{A}} \dot{\mathbf{q}}\right), \\
\ddot{\mathbf{q}} & =\overline{\mathbf{M}}^{\dagger}(\Upsilon-\overline{\mathbf{C}} \dot{\mathbf{q}}-\overline{\mathbf{N}})-\mathbf{A}^{\dagger T} \dot{\mathbf{A}} \dot{\mathbf{q}}=\lim _{\varepsilon \rightarrow 0} \overline{\mathbf{M}}_{\varepsilon}^{\dagger}(\Upsilon-\overline{\mathbf{C}} \dot{\mathbf{q}}-\overline{\mathbf{N}})-\mathbf{A}_{\varepsilon}^{\dagger T} \dot{\mathbf{A}} \dot{\mathbf{q}} \\
& =\lim _{\varepsilon \rightarrow 0}\left(\overline{\mathbf{M}}_{\varepsilon}^{-1}-\overline{\mathbf{M}}_{\varepsilon}^{-1} \mathbf{A}^{T}\left(\mathbf{A} \overline{\mathbf{M}}_{\varepsilon}^{-1} \mathbf{A}^{T}\right)^{-1} \mathbf{A} \overline{\mathbf{M}}_{\varepsilon}^{-1}\right)(\Upsilon-\overline{\mathbf{C}} \dot{\mathbf{q}}-\overline{\mathbf{N}})-\overline{\mathbf{M}}_{\varepsilon}^{-1} \mathbf{A}^{T}\left(\mathbf{A} \overline{\mathbf{M}}_{\varepsilon}^{-1} \mathbf{A}^{T}\right)^{-1} \dot{\mathbf{A}} \dot{\mathbf{q}} \\
& =\lim _{\varepsilon \rightarrow 0} \overline{\mathbf{M}}_{\varepsilon}^{-1}\left(\Upsilon-\overline{\mathbf{C}} \dot{\mathbf{q}}-\overline{\mathbf{N}}-\mathbf{A}^{T}\left(\left(\mathbf{A} \overline{\mathbf{M}}_{\varepsilon}^{-1} \mathbf{A}^{T}\right)^{-1}\left(\mathbf{A} \overline{\mathbf{M}}_{\varepsilon}^{-1}(\Upsilon-\overline{\mathbf{C}} \dot{\mathbf{q}}-\overline{\mathbf{N}})+\dot{\mathbf{A}} \dot{\mathbf{q}}\right)\right)\right) \\
& =\lim _{\varepsilon \rightarrow 0} \overline{\mathbf{M}}_{\varepsilon}^{-1}\left(\Upsilon-\overline{\mathbf{C}} \dot{\mathbf{q}}-\overline{\mathbf{N}}-\mathbf{A}^{T} \lambda\right),
\end{aligned}
$$

where (125) and (129) are identically equal to the desired formulation of (17) and (18) when $\overline{\mathbf{M}}_{0}$ is non-singular. 


\section{B.3 Proof of Lemma 8}

Proof. Recall that $\lim _{\varepsilon \rightarrow 0} \overline{\mathbf{M}}_{\varepsilon}=\overline{\mathbf{M}}$ and that $\overline{\mathbf{M}}_{\varepsilon}$ is invertible for all $\varepsilon \in(0, \bar{\varepsilon})$, for some $\bar{\varepsilon}>0$. For all $\varepsilon \geq 0$, define $\overline{\mathbf{M}}_{\varepsilon}^{\dagger}$, $\mathbf{A}_{\varepsilon}^{\dagger}$, and $\Lambda_{\varepsilon}$ by replacing $\overline{\mathbf{M}}$ with $\overline{\mathbf{M}}_{\varepsilon}$ in (8). Then using equation (15) we rewrite the impulse (where all constraints $\mathbf{A}$ are taken to be for the target contact mode $J$ ),

$$
\mathbf{P}_{\lambda}=-\Lambda \mathbf{A} \dot{\mathbf{q}}^{-}=\lim _{\varepsilon \rightarrow 0}-\Lambda_{\varepsilon} \mathbf{A} \dot{\mathbf{q}}^{-}=\lim _{\varepsilon \rightarrow 0}\left(\mathbf{A} \overline{\mathbf{M}}_{\varepsilon}^{-1} \mathbf{A}^{T}\right)^{-1} \mathbf{A} \dot{\mathbf{q}}^{-},
$$

which is identically equal to (26) when $\overline{\mathbf{M}}_{0}$ is non-singular.

\section{Differential Topology}

Let $M$ be a $C^{r}$ manifold with boundary where $r \in \mathbb{N} \cup\{\infty, \omega\}$. There are several natural constructions associated with $M$ we invoke repeatedly, so we briefly introduce them here and refer the reader to Lee (2012) for formal definitions. At every point $x \in M$ there is an associated tangent space $T_{x} M$, which is a vector space with the same dimension as $M$, i.e. $\operatorname{dim} T_{x} M=\operatorname{dim} M$; if $M$ is a submanifold in a Euclidean space of suitable dimension, $T_{x} M$ may be regarded as a hypersurface in the ambient Euclidean space. Collating these tangent spaces yields the tangent bundle $T M=\bigsqcup_{x \in M}$ $T_{x} M$, which is naturally a $C^{r}$ manifold with boundary whose dimension is twice that of $M$, i.e. $\operatorname{dim} T M=2 \operatorname{dim} M$. There is a canonical projection $\pi: T M \rightarrow M$ that simply "forgets" the tangent vector portion of a point $(x, v) \in T M$, i.e. $\pi(x, v)=x$. At every point $x \in M$ there is an associated cotangent space $T_{x}^{*} M$, which is the dual of the vector space $T_{x} M$ (i.e. every $v \in T_{x}^{*} M$ is a linear operator $v: T_{x} M \rightarrow \mathbb{R}$ ). Collating these cotangent spaces yields the cotangent bundle $T^{*} M=\bigsqcup_{x \in M} T_{x}^{*} M$, which is naturally a $C^{r}$ manifold with boundary whose dimension is twice that of $M$, i.e. $\operatorname{dim} T^{*} M=2 \operatorname{dim} M$. A $C^{r}$ map $F: M \rightarrow T M$ is called a vector field if $\pi \circ F=\mathbf{I d}_{M}$ where $\pi: T M \rightarrow M$ is the canonical projection and Id $: M \rightarrow M$ is the canonical identity function. Given a $C^{1}$ map $f: M \rightarrow N$ between $C^{r}$ manifolds, there is an associated pushforward map $D f: T M \rightarrow T N$ between their tangent spaces that evaluates to a linear map $D f(x): T_{x} M \rightarrow T_{f(x)} N$ at every $x \in M$; in coordinates, $D f$ is the familiar Jacobian derivative of $f$. The rank of $f$ at $x \in M$ is defined to be the rank of the linear operator $D f(x)$; if the rank of $f$ does not vary over $M$, it is called constant rank.

By the Whitney Embedding Theorem (Lee 2012, Thm. 6.15) (if $r \in \mathbb{N} \cup\{\infty\}$ ) or the Nash Embedding Theorem (Nash 1966) (if $r=\omega$ ), $M$ admits a $C^{r}$ embedding $\imath: M \hookrightarrow \mathbb{R}^{2 n+1}$; thus any $C^{r}$ manifold may be regarded as a submanifold of a Euclidean space of suitably high dimension. Since $F$ is $C^{r}$, the pushforward $D \imath \circ F$ admits a $C^{r}$ extension $\widetilde{F}: \widetilde{M} \rightarrow T \widetilde{M}$ over an open neighborhood $\widetilde{M} \subset \mathbb{R}^{2 n+1}$ of the embedded image of $M$. The Fundamental Theorem on Flows (Lee 2012, Thm. 9.12) implies there exists a maximal flow $\widetilde{\Phi} \in C^{r}(\widetilde{\mathcal{O}}, \widetilde{M})$ for $\widetilde{\widetilde{F}}$ where $\widetilde{\mathcal{O}} \subset \mathbb{R} \times \widetilde{M}$ is the maximal flow domain. We may restrict $\widetilde{\Phi}$ to obtain a flow over $M$ as follows. For each $x \in M$, let,

$$
\begin{aligned}
& a_{x}=\inf \{t \leq 0 \mid(t, \imath(x)) \in \widetilde{\mathcal{O}} \wedge \forall s \in(t, 0]: \widetilde{\Phi}(s, \imath(x)) \in \imath(M)\}, \\
& b_{x}=\sup \{t \geq 0 \mid(t, \imath(x)) \in \widetilde{\mathcal{O}} \wedge \forall s \in[0, t): \widetilde{\Phi}(s, \imath(x)) \in \imath(M)\} .
\end{aligned}
$$

Let $T_{x} \subset \mathbb{R}$ be the interval between $a_{x}$ and $b_{x}$, including the endpoint if the corresponding infimum or supremum is achieved. Then let $\mathcal{O}=\bigcup_{x \in M} T_{x} \times\{x\} \subset \mathbb{R} \times M$ and, noting that $\widetilde{\Phi}(t, \imath(x)) \in \imath(M)$ if $(t, x) \in \mathcal{O}$, define the flow $\Phi: \mathcal{O} \rightarrow M$ by $\Phi(t, x)=\imath^{-1}(\widetilde{\Phi}(t, \imath(x)))$. Note that $\Phi$ is $C^{r}$ in the sense that $l \circ \Phi$ admits a $C^{r}$ extension, $\widetilde{\Phi}$.

For any $G \subset \partial M$, let,

$$
H=\{x \in M \mid \exists t \geq 0:(t, x) \in \mathcal{O} \wedge \Phi(t, x) \in G\} .
$$

Define $\eta: H \rightarrow \mathbb{R}$ by,

$$
\forall x \in H: \eta(x)=\inf \{t \geq 0 \mid(t, x) \in \mathcal{O} \wedge \Phi(t, x) \in G\},
$$

and $\psi: H \rightarrow G$ by $\psi(x)=\Phi(\eta(x), x)$ for all $x \in H$. Letting $\widetilde{H}=\left\{x \in H: F(\psi(x)) \notin T_{\psi(x)} \partial M\right\}$, it is clear that $\left.\eta\right|_{\widetilde{H}} \in$ $C^{r}(\widetilde{H}, \mathbb{R})$. Note that $\eta$ is not differentiable at any point $x \in H$ for which $F(\psi(x)) \in T_{\psi(x)} \partial M$; changing coordinates to a flowbox makes this obvious. Intuitively, the impact time has unbounded sensitivity to initial conditions near such a point of tangency. 


\section{Hybrid Differential Topology}

In mechanical systems undergoing intermittent contact with the environment (i.e., terrain or objects), the dynamics are "piecewise-defined" (or hybrid): whenever a limb attaches or detaches from the substrate there is an instantaneous change in the set of active constraints, leading in general to a discontinuous change in velocity and (constraint) force. Though it is possible to analyze these discontinuous dynamics in the ambient tangent bundle as in Ballard (2000), introducing a distinct portion of state space associated with every contact mode renders both the continuoustime dynamics (given by the flow of a vector field) and discrete-time dynamics (specified by a reset map) smooth. Thus although additional notational overhead is required to index the constituent dynamical elements, the extra effort is partially compensated by enabling the use of elementary constructions from differential topology (rather than sophisticated measure-theoretic techniques used in Ballard (2000)).

Motivated largely by these observations, Burden et al. (2015b) proposed to define hybrid dynamical systems over a finite disjoint union,

$$
M=\bigsqcup_{J \in \mathcal{J}} M_{J}=\bigcup_{J \in \mathcal{J}}\{J\} \times M_{J}=\left\{(J, x): J \in \mathcal{J}, x \in M_{J}\right\},
$$

where $M_{J}$ is a finite dimensional $C^{r}$ manifold (possibly with corners) for each $J \in \mathcal{J}$. By endowing $M$ with the unique largest topology with respect to which the (canonical) inclusions $M_{J} \hookrightarrow M$ are continuous (Lee 2012, Prop. A.25), the set $M$ becomes a second-countable, Hausdorff topological space which is locally Euclidean in the sense that each point $x \in M$ has a neighborhood that is homeomorphic to an open subset of $\mathbb{R}^{n_{x}}$, some $n_{x} \in \mathbb{N}$. Since the dimension is no longer required to be fixed, $M$ is technically not a topological manifold (Lee 2012, Chapter 1). However, it is a mild generalization ${ }^{26}$, hence we refer to it as a hybrid topological manifold.

Motivated by the self-manipulation system from Section 3, we extend the definition in Burden et al. (2015b) to allow the component manifolds $M_{J}$ to possess corners. Unfortunately there is not presently a consensus on what ought to be the definition of a manifold with corners (Joyce 2012, Remark 2.11). Fortunately, for our purposes the most straightforward definition in (Lee 2012, Ch. 16) suffices. This variant, for instance, ensures smooth extensibility of maps between manifolds with corners; see the bottom paragraph of (Lee 2012, p. 27). (Note that the discussion of manifolds with boundary in (Hirsch 1976, Sec. 1.4) (termed $\partial$-manifolds) does not address this, though (Hirsch 1976, Lem. 3.1 in Sec. 2.3) should make it unsurprising.) This coincides with (Joyce 2012, Def. 1, 2).

For each $J \in \mathcal{J}, M_{J}$ has an associated maximal $C^{r}$ atlas $\mathcal{A}_{J}$. We construct a maximal $C^{r}$ hybrid atlas for $M$ by collecting charts from the atlases on the components of $M$ :

$$
\mathcal{A}=\left\{\left(\{J\} \times U, \varphi \circ \pi_{J}\right): J \in \mathcal{J},(U, \varphi) \in \mathcal{A}_{J}\right\},
$$

where $\pi_{J}:\{J\} \times M_{J} \rightarrow M_{J}$ is the canonical projection. We refer to the pair $(M, \mathcal{A})$ as a $C^{r}$ hybrid manifold, but may suppress the atlas when it is clear from context. We define the hybrid tangent bundle as the disjoint union of the component tangent bundles,

$$
T M=\coprod_{J \in \mathcal{J}} T M_{J},
$$

and the hybrid boundary as the disjoint union of the boundaries,

$$
\partial M=\coprod_{J \in \mathcal{J}} \partial M_{J} .
$$

Let $M=\amalg_{J \in \mathcal{J}} M_{J}$ and $N=\amalg_{L \in \mathcal{L}} N_{L}$ be two hybrid manifolds. Note that if a map $R: M \rightarrow N$ is continuous as a map between topological spaces, then for each $J \in \mathcal{J}$ there exists $L \in \mathcal{L}$ such that $R\left(M_{J}\right) \subset N_{L}$ and hence $\left.R\right|_{M_{J}}: M_{J} \rightarrow N_{L}$. Using this observation, we define differentiability for continuous maps between hybrid manifolds. Namely, a map $R: M \rightarrow N$ is called $C^{r}$ if $R$ is continuous and $\left.R\right|_{M_{J}}: M_{J} \rightarrow N$ is $C^{r}$ for each $J \in \mathcal{J}$. In this case the hybrid pushforward $D R: T M \rightarrow T N$ is the $C^{r}$ map defined piecewise as $\left.D R\right|_{T M_{J}}=D\left(\left.R\right|_{M_{J}}\right)$ for each $J \in \mathcal{J}$. A $C^{r}$ map $F: M \rightarrow T M$ is called a hybrid vector field if $\pi \circ F=\mathbf{I d}_{M}$ where $\pi: T M \rightarrow M$ is the canonical projection and $\mathbf{I d}: M \rightarrow M$ is the canonical identity function.

\section{E Proofs supporting Theorem 10}

\section{E.1 Proof that velocity is bounded}

The following are standard results used in the proof of Theorem 10 to prove that velocity is bounded. For convenience, we transcribe and apply to our setting these statements from Ballard (2000), which applies them to achieve a similar aim, however this is not to imply that Ballard (2000) is necessarily the original source of these results.

\footnotetext{
${ }^{26}$ Since, crucially, each of the distinct finite components $M_{J}$ is a conventional smooth $C^{r}$ manifold (of necessarily fixed dimension).
} 
Lemma 11 (Ballard 2000, Prop. 7). If $\left(\widetilde{\mathbf{q}}, \dot{\mathbf{q}}^{+}\right):[\underline{t}, \bar{t}) \rightarrow T \mathcal{Q}$ is a right-continuous trajectory of a Lagrangian system subject to perfect unilateral constraints with efforts map $\mathbf{f}:=\Upsilon-\overline{\mathbf{N}}$, then for all $t \in[\underline{t}, \bar{t})$ :

$$
\frac{1}{2}\left|\dot{\mathbf{q}}^{+}(t)\right|_{\overline{\mathbf{M}}}^{2}-\frac{1}{2}\left|\dot{\overrightarrow{\mathbf{q}}}^{+}(\underline{t})\right|_{\overline{\mathbf{M}}}^{2} \leq \int_{\underline{t}}^{t} \mathbf{f}\left(\widetilde{\mathbf{q}}(s), \dot{\overrightarrow{\mathbf{q}}}^{+}(s)\right) \dot{\overrightarrow{\mathbf{q}}}^{+}(s) d s
$$

Lemma 12 (Ballard 2000, Lem. 17). Let $a:[\underline{t}, \bar{t}] \rightarrow \mathbb{R}$ be integrable and nonnegative for almost all $t \in(\underline{t}, \bar{t})$. If $\phi:[\underline{t}, \bar{t}] \rightarrow \mathbb{R}$ has bounded variation and,

$$
\forall t \in[\underline{t}, \bar{t}]: \frac{1}{2} \phi^{2}(t) \leq \frac{1}{2} \alpha^{2}+\int_{\underline{t}}^{t} a(s) \phi(s) d s,
$$

for some $\alpha \geq 0$ then,

$$
\forall t \in[\underline{t}, \bar{t}]:|\phi(t)| \leq \alpha+\int_{\underline{t}}^{t} a(s) d s .
$$

Lemma 13 (Ballard 2000, Lem. 15; Sastry 1999, Prop. 3.21). Let $a_{1}:[\underline{t}, \bar{t}] \rightarrow \mathbb{R}$ have bounded variation and $a_{2}$ : $[\underline{t}, \bar{t}] \rightarrow \mathbb{R}$ be integrable and nonnegative for almost all $t \in(\underline{t}, \bar{t})$. If $\phi:[\underline{t}, \bar{t}] \rightarrow \mathbb{R}$ has bounded variation and,

$$
\forall t \in[\underline{t}, \bar{t}]: \phi(t) \leq a_{1}(t)+\int_{\underline{\underline{t}}}^{t} a_{2}(s) \phi(s) d s
$$

then,

$$
\forall t \in[\underline{t}, \bar{t}]: \phi(t) \leq a_{1}(t)+\int_{\underline{t}}^{t} a_{1}(s) a_{2}(s) \exp \left(\int_{s}^{t} a_{2}(\sigma) d \sigma\right) d s .
$$

We apply the Lemmas above as in the proof of (Ballard 2000, Thm. 10) to establish that the velocity is bounded on finite time horizons. Let $\left(\widetilde{\mathbf{q}}^{+}, \dot{\widetilde{\mathbf{q}}}\right):[\underline{t}, \bar{t}) \rightarrow T \mathcal{Q}$ be a right-continuous trajectory of a Lagrangian system subject to perfect unilateral constraints and with forces that satisfies the bound in (19). Lemma 11 yields for all $t \in T:=[\underline{t}, \bar{t})$ :

$$
\frac{1}{2}\left|\dot{\widetilde{\mathbf{q}}}^{+}(t)\right|_{\overline{\mathbf{M}}}^{2}-\frac{1}{2}\left|\dot{\overrightarrow{\mathbf{q}}}^{+}(\underline{t})\right|_{\overline{\mathbf{M}}}^{2} \leq \int_{\underline{t}}^{t} \mathbf{f}\left(\widetilde{\mathbf{q}}(s), \dot{\overrightarrow{\mathbf{q}}}^{+}(s)\right) \dot{\overrightarrow{\mathbf{q}}}^{+}(s) d s
$$

Applying Lemma 12 with $\phi(t)=\left|\dot{\tilde{\mathbf{q}}}^{+}(t)\right|_{\overline{\mathbf{M}}}, \alpha=\left|\dot{\tilde{\mathbf{q}}}^{+}(\underline{t})\right|_{\overline{\mathbf{M}}}$, and $a(s)=\left|\mathbf{f}\left(\widetilde{\mathbf{q}}(s), \dot{\mathbf{q}}^{+}(s)\right)\right|_{\overline{\mathbf{M}}^{-1}}$ combined with (19) implies for $t \in T$ :

$$
\begin{aligned}
\left|\dot{\widetilde{\mathbf{q}}}^{+}(t)\right|_{\overline{\mathbf{M}}} & \leq\left|\dot{\widetilde{\mathbf{q}}}^{+}(\underline{t})\right|_{\overline{\mathbf{M}}}+\int_{\underline{t}}^{t}\left|\mathbf{f}\left(\widetilde{\mathbf{q}}(s), \dot{\widetilde{\mathbf{q}}}^{+}(s)\right)\right|_{\overline{\mathbf{M}}^{-1}} d s \\
& \leq\left|\dot{\dot{\mathbf{q}}}^{+}(\underline{t})\right|_{\overline{\mathbf{M}}}+\int_{\underline{t}}^{t} C\left[1+\left|\dot{\mathbf{q}}^{+}(s)\right|_{\overline{\mathbf{M}}}+d d_{\overline{\mathbf{M}}}(\widetilde{\mathbf{q}}(\underline{t}), \widetilde{\mathbf{q}}(s))\right] d s .
\end{aligned}
$$

Recalling that $d_{\overline{\mathbf{M}}}(\widetilde{\mathbf{q}}(\underline{t}), \widetilde{\mathbf{q}}(t)) \leq \int_{\underline{t}}^{t}\left|\dot{\overrightarrow{\mathbf{q}}}^{+}(s)\right|_{\overline{\mathbf{M}}} d s$ we find,

$$
d_{\overline{\mathbf{M}}}(\widetilde{\mathbf{q}}(\underline{t}), \widetilde{\mathbf{q}}(t))+\left|\dot{\mathbf{q}}^{+}(t)\right|_{\overline{\mathbf{M}}} \leq\left|\dot{\mathbf{q}}^{+}(\underline{t})\right|_{\overline{\mathbf{M}}}+C(t-\underline{t})+\int_{\underline{t}}^{t}(1+C)\left[\left|\dot{\mathbf{q}}^{+}(s)\right|_{\overline{\mathbf{M}}}+d_{\overline{\mathbf{M}}}(\widetilde{\mathbf{q}}(\underline{t}), \widetilde{\mathbf{q}}(s))\right] d s .
$$

Applying Lemma 13 with $\phi(t)=d_{\overline{\mathbf{M}}}(\widetilde{\mathbf{q}}(\underline{t}), \widetilde{\mathbf{q}}(t))+\left|\dot{\mathbf{q}}^{+}(t)\right|_{\overline{\mathbf{M}}}, a_{1}(s)=\left|\dot{\mathbf{q}}^{+}(\underline{t})\right|_{\overline{\mathbf{M}}}+C(t-\underline{t}), a_{2}(s)=1+C$ yields,

$$
d_{\overline{\mathbf{M}}}(\widetilde{\mathbf{q}}(\underline{t}), \widetilde{\mathbf{q}}(t))+\left|\dot{\overrightarrow{\mathbf{q}}}^{+}(t)\right|_{\overline{\mathbf{M}}} \leq a_{1}(t)+\int_{\underline{t}}^{t} a_{1}(s) a_{2}(s) \exp \left[\int_{s}^{t} a_{2}(\sigma) d \sigma\right] d s
$$

In particular, since the right-hand-side of the inequality is bounded on finite time horizons, velocity is also bounded on finite time horizons,

$$
\bar{v}:=\sup _{t \in T}\left\{\left|\dot{\mathbf{q}}^{+}(t)\right| \overline{\mathbf{M}}\right\}<\infty .
$$




\section{E.2 Integration-by-Parts}

Suppose $(\mathbf{q}, \dot{\mathbf{q}}):[\underline{t}, t] \rightarrow T \mathcal{Q}$ satisfies (17). Then left-multiplying by $\overline{\mathbf{M}}$ and rearranging,

$$
\overline{\mathbf{M}}(\mathbf{q}) \ddot{\mathbf{q}}+\overline{\mathbf{C}}(\mathbf{q}, \dot{\mathbf{q}}) \dot{\mathbf{q}}=\Upsilon(\mathbf{q}, \dot{\mathbf{q}})-\overline{\mathbf{N}}(\mathbf{q}, \dot{\mathbf{q}})-\mathbf{A}^{T}(\mathbf{q}) \lambda(\mathbf{q}, \dot{\mathbf{q}}),
$$

where for all $i, j \in\{1, \ldots, \mathrm{q}\}$,

$$
\overline{\mathbf{C}}_{i j}(\mathbf{q}, \dot{\mathbf{q}}):=\sum_{k=1}^{\mathrm{q}} \frac{1}{2}\left(\frac{\partial \overline{\mathbf{M}}_{i j}(\mathbf{q})}{\partial \mathbf{q}^{k}}+\frac{\partial \overline{\mathbf{M}}_{i k}(\mathbf{q})}{\partial \mathbf{q}^{j}}-\frac{\partial \overline{\mathbf{M}}_{k j}(\mathbf{q})}{\partial \mathbf{q}^{i}}\right) \dot{\mathbf{q}}^{k},
$$

see (Murray et al. 1994, Eqn. 4.23) or (Johnson and Koditschek 2013a, Eqn. 30) for details. Note that for all $i \in$ $\{1, \ldots, \mathrm{q}\}$,

$$
\begin{aligned}
& {[\overline{\mathbf{M}} \ddot{\mathbf{q}}+\overline{\mathbf{C}} \dot{\mathbf{q}}]^{i}=\sum_{j=1}^{\mathrm{q}}\left[\overline{\mathbf{M}}_{i j} \ddot{\mathbf{q}}^{j}\right]+\sum_{j, k=1}^{\mathrm{q}}\left[\frac{1}{2}\left(\frac{\partial \overline{\mathbf{M}}_{i j}(\mathbf{q})}{\partial \mathbf{q}^{k}}+\frac{\partial \overline{\mathbf{M}}_{i k}(\mathbf{q})}{\partial \mathbf{q}^{j}}-\frac{\partial \overline{\mathbf{M}}_{k j}(\mathbf{q})}{\partial \mathbf{q}^{i}}\right) \dot{\mathbf{q}}^{k} \dot{\mathbf{q}}^{j}\right]} \\
& =\sum_{j=1}^{\mathrm{q}}\left[\overline{\mathbf{M}}_{i j} \ddot{\mathbf{q}}^{j}\right]+\sum_{j, k=1}^{\mathrm{q}}\left[\left(\frac{\partial \overline{\mathbf{M}}_{i j}(\mathbf{q})}{\partial \mathbf{q}^{k}}-\frac{1}{2} \frac{\partial \overline{\mathbf{M}}_{k j}(\mathbf{q})}{\partial \mathbf{q}^{i}}\right) \dot{\mathbf{q}}^{k} \dot{\mathbf{q}}^{j}\right] \\
& =\frac{d}{d t} \sum_{j=1}^{\mathrm{q}}\left[\overline{\mathbf{M}}_{i j} \dot{\mathbf{q}}^{j}\right]-\sum_{j, k=1}^{\mathrm{q}}\left[\frac{1}{2} \frac{\partial \overline{\mathbf{M}}_{k j}(\mathbf{q})}{\partial \mathbf{q}^{i}} \dot{\mathbf{q}}^{k} \dot{\mathbf{q}}^{j}\right]=\frac{d}{d t} \sum_{j=1}^{\mathrm{q}}\left[\overline{\mathbf{M}}_{i j} \dot{\mathbf{q}}^{j}\right]+\widetilde{\mathbf{C}}^{i}(\mathbf{q}, \dot{\mathbf{q}}),
\end{aligned}
$$

where,

$$
\widetilde{\mathbf{C}}^{i}(\mathbf{q}, \dot{\mathbf{q}}):=-\frac{1}{2} \sum_{j, k=1}^{\mathrm{q}} \frac{\partial \overline{\mathbf{M}}_{k j}(\mathbf{q})}{\partial \mathbf{q}^{i}} \dot{\mathbf{q}}^{k} \dot{\mathbf{q}}^{j}
$$

Therefore rearranging (136) we have for each $i \in\{1, \ldots, \mathrm{q}\}$,

$$
\frac{d}{d t} \sum_{j=1}^{\mathrm{q}}\left[\overline{\mathbf{M}}_{i j} \dot{\mathbf{q}}^{j}\right]=\Upsilon^{i}-\overline{\mathbf{N}}^{i}-\widetilde{\mathbf{C}}^{i}-\left(\mathbf{A}^{T} \lambda\right)^{i} .
$$

Integrating both sides of (137) over the time interval $[\underline{t}, t]$, reintroducing the dependence on $(\mathbf{q}, \dot{\mathbf{q}})$ and time, and vectorizing over the index $i$,

$$
\overline{\mathbf{M}}(\mathbf{q}(t)) \dot{\mathbf{q}}(t)-\overline{\mathbf{M}}(\mathbf{q}(\underline{t})) \dot{\mathbf{q}}(\underline{t})=\int_{\underline{t}}^{t}\left(\Upsilon(\mathbf{q}(s), \dot{\mathbf{q}}(s))-\overline{\mathbf{N}}(\mathbf{q}(s), \dot{\mathbf{q}}(s))-\widetilde{\mathbf{C}}(\mathbf{q}(s), \dot{\mathbf{q}}(s))-\mathbf{A}(\mathbf{q}(s))^{T} \lambda(\mathbf{q}(s), \dot{\mathbf{q}}(s))\right) d s,
$$

as used in (90).

\section{E.3 Proof that constraint forces and impulses are bounded}

The following is a transcription of the argument used in the proof of (Ballard 2000, Prop. 18) to show that constraint forces and impulses are bounded on bounded time intervals.

Given a right-continuous trajectory $\left(\widetilde{\mathbf{q}}, \dot{\mathbf{q}}^{+}\right):[\underline{t}, \bar{t}) \rightarrow T \mathcal{Q}$ of a Lagrangian system subject to perfect unilateral constraints, we assume that: the inertia tensor $\overline{\mathbf{M}}$ is nondegenerate; the position tends to a limit $\bar{q}:=\lim _{t \rightarrow i} \widetilde{\mathbf{q}}$; and the velocity is bounded by $\bar{v}:=\sup _{t \in T}\left\{\left|\dot{\mathbf{q}}^{+}(t)\right| \overline{\mathbf{M}}_{\mathbf{M}}\right\}<\infty$ where $T=[\underline{t}, \bar{t})$. This ensures there exists a compact neighborhood $K \subset V$ such that $\widetilde{\mathbf{q}}([\underline{t}, \bar{t})) \subset K$ and hence with $\bar{B}(0, \bar{v}) \subset \mathbb{R}^{\mathrm{q}}$ denoting the closed ball of radius $\bar{v}$ centered at the origin, the compact subset $K^{\prime}:=\bar{B}(0, \bar{v}) \times K \subset T V$ contains $\left(\widetilde{\mathbf{q}}, \dot{\mathbf{q}}^{+}\right)([\underline{t}, \bar{t}))$. This implies the following constants are finite:

$$
\begin{aligned}
F & :=\max _{j \in\{1, \ldots, \mathbf{q}\}} \max _{(\mathbf{q}, \mathbf{q}) \in K^{\prime}}\left|\Upsilon^{j}(\mathbf{q}, \dot{\mathbf{q}})-\overline{\mathbf{N}}^{j}(\mathbf{q}, \dot{\mathbf{q}})\right|, \\
G & :=\max _{j, k, \ell \in\{1, \ldots, \mathbf{q}\}} \max _{\mathbf{q} \in K}\left|\frac{\partial \overline{\mathbf{M}}_{k \ell}(\mathbf{q})}{\partial \mathbf{q}^{j}}\right| .
\end{aligned}
$$


Letting $\sigma_{\max }$ and $\sigma_{\min }$ denote the maximum and minimum singular values of $\overline{\mathbf{M}}$ over $K$, we obtain the following bounds:

$$
\begin{array}{r}
\max _{j \in\{1, \ldots, \mathrm{q}\}} \max _{(\mathbf{q}, \dot{\mathbf{q}}) \in K^{\prime}}\left|\sum_{k=1}^{\mathrm{q}} \overline{\mathbf{M}}_{j k}(\mathbf{q}) \dot{\mathbf{q}}^{k}\right| \leq \sqrt{\sigma_{\max }}, \\
\max _{j \in\{1, \ldots, \mathrm{q}\}} \max _{(\mathbf{q}, \dot{\mathbf{q}}) \in K^{\prime}}\left|\dot{\mathbf{q}}^{j}\right| \leq \frac{\bar{v}}{\sqrt{\sigma_{\min }}} .
\end{array}
$$

Suppressing dependence on $\widetilde{\mathbf{q}}$ and $\dot{\mathbf{q}}^{+}$, we arrive at the bound that for each $j \in\{1, \ldots,|\mathcal{K}|\}$,

$$
-\left[\overline{\mathbf{M}}^{j}(t) \dot{\mathbf{q}}^{+}(t)-\overline{\mathbf{M}}^{j}\left(t_{\underline{m}}\right) \dot{\overrightarrow{\mathbf{q}}}^{+}\left(t_{\underline{m}}\right)\right]+\int_{t_{\underline{m}}}^{t} \Upsilon^{j}-\overline{\mathbf{N}}^{j}-\widetilde{\mathbf{C}}^{j} d s \leq 2 \sqrt{\sigma_{\max }} \overline{\mathbf{v}}+\left(F+\frac{\mathrm{q}^{2} G \bar{v}^{2}}{2 \sigma_{\min }}\right)\left(t-t_{\underline{m}}\right)<\infty,
$$

thus satisfying the condition on (93).

\section{References}

Ames, A., Zheng, H., Gregg, R. and Sastry, S. (2006). Is there life after Zeno? Taking executions past the breaking (Zeno) point, American Control Conference, 2006, pp. 2652-2657.

Andersson, R. L. (1989). Aggressive trajectory generator for a robot ping-pong player, IEEE Control Systems Magazine 9(2): 15-21.

Anitescu, M. and Potra, F. A. (1997). Formulating dynamic multi-rigid-body contact problems with friction as solvable linear complementarity problems, Nonlinear Dynamics 14(3): 231-247.

Ascher, U. M. and Petzold, L. R. (1998). Computer methods for ordinary differential equations and differentialalgebraic equations, Vol. 61, SIAM.

Back, A., Guckenheimer, J. M. and Myers, M. (1993). A dynamical simulation facility for hybrid systems, in R. Grossman, A. Nerode, A. Ravn and H. Rischel (eds), Hybrid Systems, Vol. 736 of Lecture Notes in Computer Science, Springer Berlin / Heidelberg, pp. 255-267.

Balasubramanian, R., Rizzi, A. and Mason, M. T. (2008). Legless locomotion: A novel locomotion technique for legged robots, International Journal of Robotics Research 27(5): 575-594.

Ballard, P. (2000). The dynamics of discrete mechanical systems with perfect unilateral constraints, Archive for Rational Mechanics and Analysis 154(3): 199-274.

Baraff, D. (1991). Coping with friction for non-penetrating rigid body simulation, ACM SIGGRAPH Computer Graphics, Vol. 25.4, pp. 31-41.

Bayo, E. and Ledesma, R. (1996). Augmented lagrangian and mass-orthogonal projection methods for constrained multibody dynamics, Nonlinear Dynamics 9(1-2): 113-130.

Bertsimas, D. and Tsitsiklis, J. (1997). Introduction to Linear Optimization, 1st edn, Athena Scientific, Belmont, MA.

Blickhan, R. (1989). The spring-mass model for running and hopping, Journal of biomechanics 22(11): 1217-1227.

Brill, A., De, A., Johnson, A. M. and Koditschek, D. E. (2015). Tail-assisted rigid and compliant legged leaping, Proceedings of the IEEE/RSJ International Conference on Intelligent Robots and Systems, Hamburg, Germany, pp. 6304-6311.

Brogliato, B., Ten Dam, A., Paoli, L., Genot, F. and Abadie, M. (2002). Numerical simulation of finite dimensional multibody nonsmooth mechanical systems, Applied Mechanics Reviews 55: 107.

Buehler, M., Koditschek, D. E. and Kindlmann, P. J. (1994). Planning and control of robotic juggling and catching tasks, International Journal of Robotics Research 13(2): 101-118. 
Burden, S. A., Gonzalez, H., Vasudevan, R., Bajcsy, R. and Sastry, S. S. (2015a). Metrization and simulation of controlled hybrid systems, IEEE Transactions on Automatic Control 60(9): 2307-2320.

Burden, S. A., Revzen, S. and Sastry, S. S. (2015b). Model reduction near periodic orbits of hybrid dynamical systems, IEEE Transactions on Automatic Control 60(10): 2626-2639.

Chatterjee, A. (1999). On the realism of complementarity conditions in rigid body collisions, Nonlinear Dynamics 20(2): 159-168.

Chatterjee, A., Pratap, R., Reddy, C. and Ruina, A. (2002). Persistent passive hopping and juggling is possible even with plastic collisions, International Journal of Robotics Research 21(7): 621-634.

Chatterjee, A. and Ruina, A. (1998). A new algebraic rigid-body collision law based on impulse space considerations, ASME Journal of Applied Mechanics 65: 939-951.

Collins, P. (2004). A trajectory-space approach to hybrid systems, Proceedings of the 16th International Symposium on the Mathematical Theory of Networks and Systems, p. 12.

Cottle, R. W. (1968). On a problem in linear inequalities, Journal of the London Mathematical Society 1(1): 378-384.

Cottle, R. W. (1974). Manifestations of the Schur complement, Linear Algebra and its Applications 8(3): 189-211.

Cottle, R. W. and Dantzig, G. B. (1968). Complementary pivot theory of mathematical programming, Linear Algebra and its Applications 1(1): 103-125.

Drumwright, E. (2010). Avoiding zeno's paradox in impulse-based rigid body simulation, Proceedings of the IEEE International Conference on Robotics and Automation, IEEE, pp. 5127-5132.

Drumwright, E. and Shell, D. A. (2011). Modeling contact friction and joint friction in dynamic robotic simulation using the principle of maximum dissipation, Algorithmic Foundations of Robotics IX, Springer, pp. 249-266.

Dupont, P. E. and Yamajako, S. P. (1994). Jamming and wedging in constrained rigid-body dynamics, Proceedings of the IEEE International Conference on Robotics and Automation, pp. 2349-2354.

Featherstone, R. (2008). Rigid body dynamics algorithms, Springer, New York.

Folland, G. B. (2002). Advanced calculus, Prentice-Hall, Englewood Cliffs, NJ.

Full, R. and Koditschek, D. (1999). Templates and anchors: Neuromechanical hypotheses of legged locomotion on land, Journal of Experimental Biology 202(23): 3325-3332.

Gilardi, G. and Sharf, I. (2002). Literature survey of contact dynamics modelling, Mechanism and machine theory 37(10): 1213-1239.

Goebel, R. and Teel, A. R. (2006). Solutions to hybrid inclusions via set and graphical convergence with stability theory applications, Automatica 42(4): 573-587.

Greenfield, A., Saranli, U. and Rizzi, A. A. (2005). Solving models of controlled dynamic planar rigid-body systems with frictional contact, International Journal of Robotics Research 24(11): 911-931.

Hatcher, A. (2002). Algebraic topology, Cambridge University Press, Cambridge, UK.

Heemels, W., Schumacher, J. M. and Weiland, S. (2000). Linear complementarity systems, SIAM Journal on Applied Mathematics pp. 1234-1269.

Hermann, R. (1973). Geodesics of singular Riemannian metrics, Bulletin of the American Mathematical Society 79(4): 780-782.

Hirsch, M. (1976). Differential topology, Springer-Verlag, New York.

Holmes, P., Full, R. J., Koditschek, D. E. and Guckenheimer, J. (2006). The dynamics of legged locomotion: Models, analyses, and challenges, SIAM Review 48(2): 207-304. 
Housner, G. W. (1963). The behavior of inverted pendulum structures during earthquakes, Bulletin of the Seismological Society of America 53(2): 403-417.

Huang, W. H. and Mason, M. T. (1998). Experiments in impulsive manipulation, Proceedings of the IEEE International Conference on Robotics and Automation, Vol. 2, pp. 1077-1082.

Hurmuzlu, Y. and Marghitu, D. B. (1994). Rigid body collisions of planar kinematic chains with multiple contact points, International Journal of Robotics Research 13(1): 82-92.

Ingleton, A. W. (1966). A problem in linear inequalities, Proceedings of the London Mathematical Society 3(1): 519536.

Ivanov, A. P. (1995). On multiple impact, Journal of Applied Mathematics and Mechanics 59(6): 887-902.

Jo, S. E., Kim, S.-W. and Park, T. J. (2004). Equally constrained affine projection algorithm, Conference Record of the Thirty-Eighth Asilomar Conference on Signals, Systems and Computers, Vol. 1, pp. 955-959.

Johansson, K. H., Egerstedt, M., Lygeros, J. and Sastry, S. (1999). On the regularization of Zeno hybrid automata, Systems \& Control Letters 38(3): 141-150.

Johnson, A. M. (2014). Self-Manipulation and Dynamic Transitions for a Legged Robot, $\mathrm{PhD}$ Thesis, Electrical and Systems Engineering, University of Pennsylvania, Philadelphia, PA.

Johnson, A. M. and Koditschek, D. E. (2013a). Legged self-manipulation, IEEE Access 1: 310-334.

Johnson, A. M. and Koditschek, D. E. (2013b). Toward a vocabulary of legged leaping, Proceedings of the IEEE International Conference on Robotics and Automation, pp. 2553-2560.

Joyce, D. (2012). On manifolds with corners, in J. Li and D. H. Phong (eds), Advances in Geometric Analysis, Vol. 21 of Advanced Lectures in Mathematics, International Press of Boston, Inc., pp. 225-258.

Kajita, S., Yamaura, T. and Kobayashi, A. (1992). Dynamic walking control of a biped robot along a potential energy conserving orbit, IEEE Transactions on Robotics and Automation 8(4): 431-438.

Keller, J. B. (1986). Impact with friction, Journal of Applied Mechanics 53(1): 1-5.

Khatib, O. (1983). Dynamic control of manipulators in operational space, Proc. of the Sixth CISM-IFToMM Symposium on Theory and Practice of Robots and Manipulators, New Delhi, India, pp. 1128-1131.

Kilmister, W. and Reeve, J. E. (1966). Rational Mechanics, Longman's, London.

Lalonde, J.-F., Vandapel, N. and Hebert, M. (2007). Data structures for efficient dynamic processing in 3-D, International Journal of Robotics Research 26(8): 777-796.

Lee, J. M. (1997). Riemannian manifolds: an introduction to curvature, Springer-Verlag, New York.

Lee, J. M. (2012). Introduction to smooth manifolds, Springer-Verlag, New York.

Lötstedt, P. (1982). Mechanical systems of rigid bodies subject to unilateral constraints, SIAM Journal on Applied Mathematics 42(2): 281-296.

Lu, T.-T. and Shiou, S.-H. (2002). Inverses of 2 x 2 block matrices, Computers \& Mathematics with Applications 43(1-2): 119-129.

Lygeros, J., Johansson, K. H., Simic, S. N., Zhang, J. and Sastry, S. S. (2003). Dynamical properties of hybrid automata, IEEE Transactions on Automatic Control 48(1): 2-17.

Mason, M. T. and Wang, Y. (1988). On the inconsistency of rigid-body frictional planar mechanics, Proceedings of the IEEE International Conference on Robotics and Automation, pp. 524-528.

McGeer, T. and Palmer, L. H. (1989). Wobbling, toppling, and forces of contact, American Journal of Physics 57(12): 1089-1098. 
Mirtich, B. and Canny, J. (1995). Impulse-based simulation of rigid bodies, Proceedings of the Symposium on Interactive 3D Graphics, ACM, pp. 181-ff.

Moreau, J. (1985). Standard inelastic shocks and the dynamics of unilateral constraints, in G. Del Piero and F. Maceri (eds), Unilateral Problems in Structural Analysis, Vol. 288 of International Centre for Mechanical Sciences, Springer, pp. 173-221.

Murray, R. M., Li, Z. and Sastry, S. S. (1994). A Mathematical Introduction to Robotic Manipulation, CRC Press, Boca Raton, FL.

Nash, J. (1966). Analyticity of the solutions of implicit function problems with analytic data, Annals of Mathematics 84(3): 345-355.

Or, Y. and Ames, A. (2011). Stability and completion of Zeno equilibria in lagrangian hybrid systems, IEEE Transactions on Automatic Control 56(6): 1322-1336.

Pagilla, P. R. and Yu, B. (2001). A stable transition controller for constrained robots, IEEE/ASME Transactions on Mechatronics 6(1): 65-74.

Painlevé, P. (1895). Leçons sur le frottement, Hermann, Paris.

Pang, J.-S., Trinkle, J. C. and Lo, G. (1996). A complementarity approach to a quasistatic multi-rigid-body contact problem, Computational Optimization and Applications 5(2): 139-154.

Papalambros, P. Y. and Wilde, D. J. (2000). Principles of optimal design: modeling and computation, Cambridge university press.

Pfeiffer, F. and Glocker, C. (1996). Multibody Dynamics with Unilateral Contacts, John Wiley \& Sons.

Quinn, D. D. (2005). Finite duration impacts with external forces, Journal of Applied Mechanics 72(5): 778-784.

Sastry, S. S. (1999). Nonlinear Systems: Analysis, Stability, and Control, Springer-Verlag, New York.

Schaal, S. and Atkeson, C. G. (1993). Open loop stable control strategies for robot juggling, Proceedings of the IEEE International Conference on Robotics and Automation, pp. 913-918.

Seghete, V. and Murphey, T. (2010). Variational solutions to simultaneous collisions between multiple rigid bodies, Proceedings of the IEEE International Conference on Robotics and Automation, pp. 2731-2738.

Shampine, L., Gladwell, I. and Brankin, R. (1991). Reliable solution of special event location problems for ODEs, ACM Transactions on Mathematical Software 17(1): 11-25.

Siciliano, B. and Khatib, O. (2008). Handbook of Robotics, Springer-Verlag, New York.

Simic, S. N., Johansson, K. H., Lygeros, J. and Sastry, S. (2005). Towards a geometric theory of hybrid systems, Dynamics of Continuous, Discrete and Impulsive Systems Series B: Applications and Algorithms 12(5-6): 649687.

Stewart, D. E. (1998). Convergence of a time-stepping scheme for rigid-body dynamics and resolution of painlevé's problem, Archive for Rational Mechanics and Analysis 145(3): 215-260.

Stewart, D. E. and Trinkle, J. C. (1996). An implicit time-stepping scheme for rigid body dynamics with inelastic collisions and coulomb friction, International Journal for Numerical Methods in Engineering 39(15): 2673-2691.

Tavernini, L. (1987). Differential automata and their discrete simulators, Nonlinear Analysis: Theory, Methods \& Applications 11(6): 665-683.

ten Dam, A. A. and Willems, J. C. (1997). A system theoretical framework to study unilaterally constrained mechanical systems, European Control Conference, pp. 3469-3474.

Todorov, E. (2011). A convex, smooth and invertible contact model for trajectory optimization, Proceedings of the IEEE International Conference on Robotics and Automation, IEEE, pp. 1071-1076. 
Todorov, E., Erez, T. and Tassa, Y. (2012). Mujoco: A physics engine for model-based control, Proceedings of the IEEE/RSJ International Conference on Intelligent Robots and Systems, IEEE, pp. 5026-5033.

Trinkle, J. C., Pang, J.-S., Sudarsky, S. and Lo, G. (1997). On dynamic multi-rigid-body contact problems with Coulomb friction, ZAMM-Journal of Applied Mathematics and Mechanics/Zeitschrift für Angewandte Mathematik und Mechanik 77(4): 267-279.

Trinkle, J. and Zeng, D. (1995). Prediction of the quasistatic planar motion of a contacted rigid body, IEEE Transactions on Robotics and Automation 11(2): 229-246.

van der Schaft, A. J. and Schumacher, J. (1998). Complementarity modeling of hybrid systems, IEEE Transactions on Automatic Control 43(4): 483-490.

Wampler, C. W. and Sommese, A. J. (2013). Applying numerical algebraic geometry to kinematics, in J. M. McCarthy (ed.), 21 st Century Kinematics, Springer London, pp. 125-159.

Wang, Y. and Mason, M. T. (1987). Modeling impact dynamics for robotic operations, Proceedings of the IEEE International Conference on Robotics and Automation, Vol. 4, pp. 678-685.

Wang, Y.-T. and Kumar, V. (1994). Simulation of mechanical systems with multiple frictional contacts, Journal of Mechanical Design 116(2): 571-580.

Wehage, R. and Haug, E. (1982). Dynamic analysis of mechanical systems with intermittent motion, Journal of Mechanical Design 104(4): 778-784.

Westervelt, E. R., Grizzle, J. W. and Koditschek, D. E. (2003). Hybrid zero dynamics of planar biped walkers, IEEE Transactions on Automatic Control 48(1): 42-56.

Witsenhausen, H. (1966). A class of hybrid-state continuous-time dynamic systems, IEEE Transactions on Automatic Control 11(2): 161-167.

Xiao, J. and Ji, X. (2001). Automatic generation of high-level contact state space, The International Journal of Robotics Research 20(7): 584-606.

Yilmaz, C., Gharib, M. and Hurmuzlu, Y. (2009). Solving frictionless rocking block problem with multiple impacts, Proceedings of the Royal Society A: Mathematical, Physical and Engineering Science 465(2111): 3323-3339.

Zhang, J., Johansson, K. H., Lygeros, J. and Sastry, S. (2001). Zeno hybrid systems, International Journal of Robust and Nonlinear Control 11(5): 435-451. 